II it lulal Bureau of standards

Lthrory, N.W. BIdg -

JUN 251963

NBS

Eechnical Mote

186

\title{
TABULATION OF PUBLISHED DATA ON SOVIET ELECTRON DEVICES
}

CHARLES P. MARSDEN

U. S. DEPARTMENT OF COMMERCE

NATIONAL BUREAU OF STANDARDS 


\section{THE NATIONAL BUREAU OF STANDARDS}

\section{Functions and Activities}

The functions of the National Bureau of Standards are set forth in the Act of Congress, March 3, 1901, as amended by Congress in Public Law 619, 1950. These include the development and maintenance of the national standards of measurement and the provision of means and methods for making measurements consistent with these standards; the determination of physical constants and properties of materials; the development of methods and instruments for testing materials, devices, and structures; advisory services to government agencies on scientific and technical problems; invention and development of devices to serve special needs of the Government; and the development of standard practices, codes, and specifications. The work includes basic and applied research, development, engineering, instrumentation, testing, evaluation, calibration services, and various consultation and information services. Research projects are also performed for other government agencies when the work relates to and supplements the basic program of the Bureau or when the Bureau's unique competence is required. The scope of activities is suggested by the listing of divisions and sections on the inside of the back cover.

\section{Publications}

The results of the Bureau's research are published either in the Bureau's own series of publications or in the journals of professional and scientific societies. The Bureau publishes three periodicals available from the Government Printing Office: The Journal of Research, published in four separate sections, presents complete scientific and technical papers; the Technical News Bulletin presents summary and preliminary reports on work in progress; and the Central Radio Propagation Lahoratory Iolospheric Predictions provides data for determining the best frequencies to use for radio communications throughout the world. There are also five series of nonperiodical publications: Monographs, Applicd Mathematics Series, Handbooks, Miscellancous Publications, and Technical Notes.

A complete listing of the Bureau's publications can be found in National Bureau of Standards Circular 460, Publications of the National Bureau of Standards, 1901 to June 1947 (\$1.25), and the Supplement to National Bureau of Standards Circular 460, July 1947 to June 1957 (\$1.50), and Miscellaneous Publication 240, July 1957 to June 1960 (includes Titles of Papers Published in Outside Journals 1950 to 1959) (\$2.25); available from the Superintendent of Documents, Government Printing Office, Washington 25, D.C. 


\title{
NATIONAL BUREAU OF STANDARDS Eechnical Note 186 \\ ISSUED JUNE 3, 1963
}

\section{TABULATION OF PUBLISHED DATA ON SOVIET ELECTRON DEVICES}

\author{
Charles P. Marsden
}

NBS Technical Notes are designed to supplement the Bureau's regular publications program. They provide a means for making available scientific data that are of transient or limited interest. Technical Notes may be listed or referred to in the open literature. 


\section{Foreword}

This tabulation of published data on Soviet electron devices has been prepared as part of the National Bureau of Standards Electron Devices Data Service. Established in 1948 to provide technical data on radio tubes to members of the Bureau staff, the service has since been extended to other scientists and engineers in government and industry. In the course of the program, a large volume of information on tubes, transistors, diodes, and other electron devices has been accumulated on punched cards. To make this information more readily available, a system has been worked out for automatically tabulating the data in handbook form. Previous tabulations include Tabulation of Data on Receiving Tubes, NBS Handbook 68 (1959); Tabulation of Data on Microwave Tubes, NBS Handbook 70 (1961); and Diode Source Book (published by Semiconductor Products magazine, (1961).

The present tabulation is the result of compilation efforts extending over the past four years. A11 the included information was taken from published specifications, and every effort has been made to ensure accuracy and completeness. However, the Bureau cannot assume responsibility for omissions nor for results obtained with these data.

A. V. Astin, Director. 


\section{Contents}

Page

1. Introduction. . . . . . . . . . . . . . . . . 1

2. Description of the Tabulation .............. 1

3. Figure 1 Organization of the Tabulation . . . . . . . . 2

4. Terminology Used in the Tabulation. . . . . . . . . . 3

4.1 Column Headings. . . . . . . . . . . . . 3

4.2 Bulb Size. . . . . . . . . . . . . . 3

4.3 Code .................... . . 4

Numerical Listing. . . . . . . . . . . . 8

Receiving Tubes. . . . . . . . . . . . 20

Power Tubes. . . . . . . . . . . . . . 30

Rectifier Tubes. . . . . . . . . . . . 35

Voltage Regulator Tubes. . . . . . . . . . 36

Current Regulator Tubes. . . . . . . . . . 36

Thyratrons . . . . . . . . . . . . . 37

Cathode Ray Tubes. . . . . . . . . . . . 39

Microwave Tubes. . . . . . . . . . . . 4 42

Transistors. . . . . . . . . . . . 43

Diodes - Rectifier . . . . . . . . . . . 50

Diodes - Power Rectifier . . . . . . . . . . 53

Diodes - Regulators. . . . . . . . . . . 53

Diodes - Mixer and Detector. . . . . . . . . 54

Diodes - Photoconductive . . . . . . . . . . 55

Phototubes and Photo multipliers . . . . . . . 58

Flash Tubes. . . . . . . . . . . . 60

Thermocouple Tubes . . . . . . . . . . 60

Thermistors. . . . . . . . . . . . . 61

Strobotrons. . . . . . . . . . . . . 62

Counter Tubes. . . . . . . . . . . . . 63

Discharge Tubes. . . . . . . . . . . . . 64

Decatrons. . . . . . . . . . . . . . 64

Light Amplifiers . . . . . . . . . . . . 64

Basing Connections . . . . . . . . . . . 65 


\section{FIG. I}

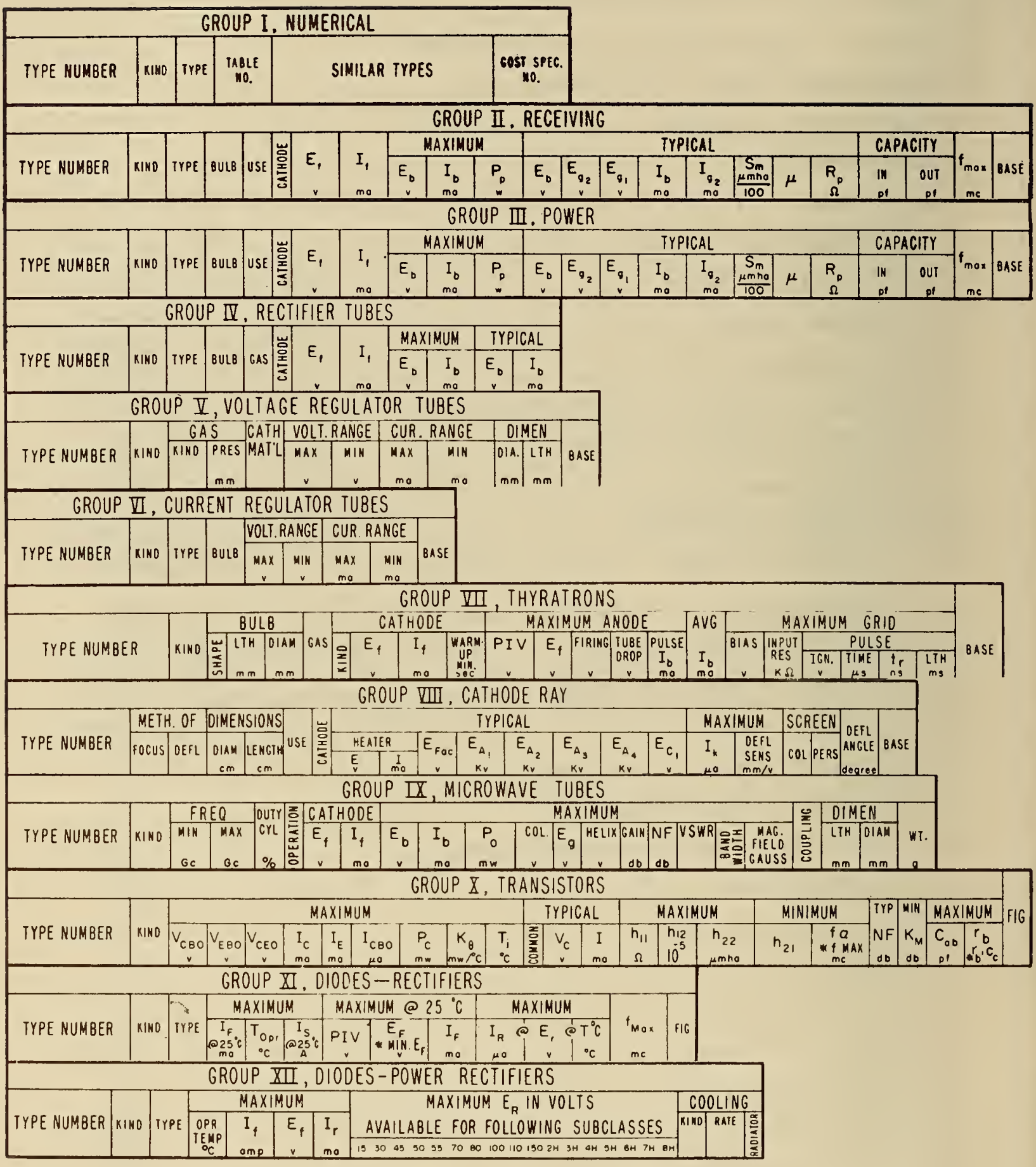




\section{FIG. I CONT'D}

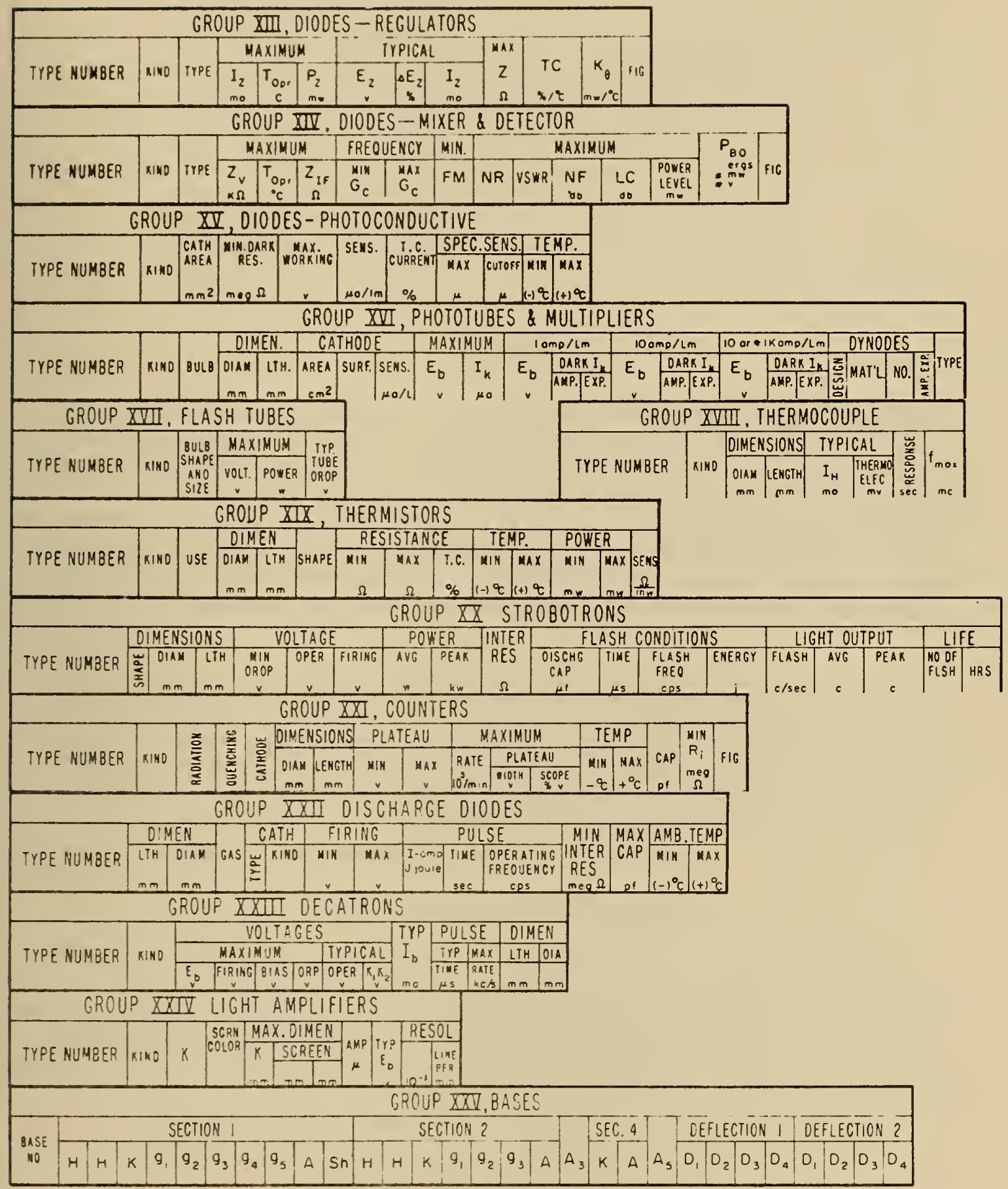



Tabulation of Published Data on Soviet Electron Devices

\section{Charles P. Marsden}

This tabulation includes published data on Soviet electron devices as collected from various publications, mostly handbooks published by the various ministries and institutes of the USSR. Information is given on a 11 active devices ranging from receiving to microwave devices, semiconductor devices, and various miscellaneous devices such as, for example, photographic flash tubes and thermistors.

\section{Introduction}

The increased circulation of published literature from the USSR and the importation of Soviet equipment has created a need for factua 1 information on Soviet electron devices. To satisfy this need, the National Bureau of Standards Electron. Devices Data Service has prepared the present tabulation in a format that could be reproduced directly from punched cards. The advantages of the use of these cards include the elimination of errors possible with other methods of reproduction, flexibility in adding or deleting information, and uniform columnar presentation.

The sources of the data are the various publications produced in the USSR and include books published by the various ministries, and technica 1 magazines. This information has been intercompared and correlated to eliminate errors and thus assure that this tabulation is as accurate as possible. Because of this intercomparison, references for the data are not given, as data for any one type of device may have been derived from several sources.

\section{Description of the Tabulation}

In each group the type numbers are arranged in alpha-numerical order in which the first numerical part of the type number is the prime sorting means. Alphabetical prefixes are the secondary sorting. means and alphabetical postfixes are the tertiary means. For example in the numerical 1ist, these type numbers will be found in the following order:

$\begin{array}{cr}\mathrm{V} 1-0.1 / 40 & \mathrm{SG} 2 \mathrm{~S} \\ \mathrm{VT} 1 & \mathrm{TO}-2 \\ 1 \mathrm{~A} 2 \mathrm{P} & 2 \mathrm{~A} 1\end{array}$


Alphabetical sorting is performed according to the English alphabet rather than the Russian which was transliterated according to the recommended practice of the Library of Congress as shown below:

$\begin{array}{llllll}\text { A } & \text { A } & \text { K } & \text { K } & \text { T } & \text { T } \\ \text { B } & \text { B } & \text { JI } & \text { L } & \text { y } & \text { U } \\ \text { B } & \text { V } & \text { Ji } & \text { M } & \Phi & \text { F } \\ \Gamma & \text { G } & \text { H } & \text { N } & \text { X } & \text { Kh } \\ \text { L } & \text { D } & \text { O } & \text { O } & \text { L } & \text { Ts } \\ \text { E } & \text { Ye } & \text { II } & \text { P } & \text { II } & \text { Sh } \\ \text { W } & \text { Zh } & \text { P } & \text { R } & \text { J } & \text { E } \\ \text { U } & \text { I } & \text { C } & \text { S } & & \end{array}$

This transliteration was necessary to put the information on punched cards and it is believed that it will cause little difficulty in use.

3. Organization of the Tabulation

The tabulation is divided into 24 groups, each with a different format and different columnar headings so that the maximum pertinent data may be included. These groups and their column headings are shown in Figure 1.

Group $I$ is a numerica 1 listing of a 11 type numbers in the complete tabulation and also includes discontinued and absolete types. A11 these types are defined by the same three-letter code to indicate the kind and type of tube. Furthermore, under the heading "Group No.", Roman numerals are used to show the group number under which the data for a type will be found. In the last column, the GOST (State National Standard) Specification Number (followed by the year of publication of the specification) is shown for the type number. These specifications include the information in and follow the format of the domestic military specifications.

This group is also an interchangeability list and known similar types are shown. Further, by means of the following symbol code, the manufacturing area and the obsolescence of the type are indicated.

* Domestic manufacture
\# European
a Russian
a Obsolete or inactive

The above definitions of these symbols are pertinent only to their use in Group I. Due to the limitation of available symbols on 1isting equipment, these same symbols are used in the other groups but are then defined as shown at the end of the definitions under the paragraph entitled "Code" (p.4).

The other groups have titles describing the particular class of. 
devices listed therein. As mentioned previously, the individual type numbers are arranged in the same alpha-numerical order.

Under each heading of the group format, the unit of measurement most common for the characteristic is shown. For example under the heading of Maximum Plate Current ( $I$ ), the unit in the heading is ma (milliamperes). However, where the $\mathrm{p}_{\text {data }}$ are in amperes, the value will be tabulated with the number followed by the letter "A", e.g., 15A. A11 these changes of units are included in the list of alphabetical symbols under code on pages 3 to 5 .

A blank in any column indicates that no value was given in the available data.

Group XXIV, "Bases", lists the basing connections for the particular "Base No." of the previous groups by a system compatible with punched cards.

Instead of the usual base diagram or line drawing, the number of each base pin is given in the column under the symbol of the electrode. This system was developed because many of the Soviet types have base connections which do not conform to the standard base designations of the Electronic Industries Association. In those instances where an electrode is connected to more than one base pin, only the lowest numbered pin is shown in the tabulation.

Outline drawings are shown for the semiconductor diodes and transistors. Similarly photographic outlines are included for counter tubes.

\section{Terminology used in the Tabulation}

\subsection{Column Headings}

The headings used in the various formats are the standard symbols as defined by the Institute of Radio Engineers or descriptive words for the characteristics. They are not further defined due either to the difficulties of translation or lack of definite information.

\subsection{Bulb Size}

This column heading, which is used in the Receiving, Power, Rectifier, etc. Groups, uses a special code to describe the bulb shape and size. The numerical part of the code indicates the diameter of the glass bulb or metal anode (power tubes) in eighths of an inch according to the American standard. The alphabetical part of the code is explained on the following page. 
A - Air-cooled anode

C - Ceramic construction

G - Globe-shaped bulb

F - Flat top of Soviet design

$\mathrm{H}$ - Helix-shaped flash tube

M - Meta 1 tube

$\mathrm{P}$ - Spira 1

R - Ring-shaped

S - ST design, i.e., the domed conical shaped glass bulb

$T$ - Cylindrica 1 shape

U - U-shape flash tube

W - Water-cooled anode

For example, a "T $3 F$ " would be a cylindrical bulb with a flat press and having a diameter of $3 / 8$ inch.

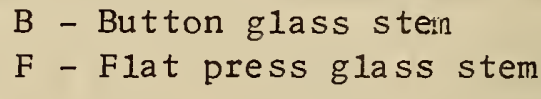

\subsection{Code}

Due to the limitations of available columns in the punched card, one- to three-letter codes have been liberally developed and used in the tabulation. These have been chosen to be readily understood. The following table lists the definitions of this code for al1 groups in alphabetical order.

Code

A Change of unit to amperes

ACO Acorn tube

AF \{Audio frequency

$\left\{\begin{array}{l}\text { Forced air cooling } \\ \text { fir }\end{array}\right.$

AHE Argon-helium gas-filled

AHN Argon-helium-neon gas-filled

AKN Argon-krypton gas-filled

AMK Aluminum-Magnesium a11oy with potassium surface

AN Natural air cooling

AO Argon-oxygen gas-filled
AR Argon gas-filled

ARC Arc rectifier - Mercury pool

BA Barium (meta1) cathode

BAG Beta and gamma radiation

BAL Ballast or current regulator

BAO Barium oxide cathode

BEA $\left\{\begin{array}{l}\text { Beam pentode } \\ \text { With beam pentode, e.g. }\end{array}\right.$ triode beam pentode

BET Beta radiation

BL Blue luminescence 
C

Circular dynode arrangement

Common collector operation

Cold cathode

Continuous wave operation

CAMI Copper-aluminum-magnesium

$\mathrm{CN}$ Converter

$\operatorname{COM}\left\{\begin{array}{l}\text { Comutator tubes } \\ \text { Compensation of temperature }\end{array}\right.$ thermistors

CON Temperature control

COU Counter tube

CP Cap, external in tabulation of bases

CS Cesium photo surface

CSB Cesium antimony photo surface

CU Copper cathode - counter tube

CYL Cylindrical shape (Thermistors)

DBA Double anode beam pentode

DEC Decatron

DET Detector operation

DIO $\left\{\begin{array}{l}\text { Diode } \\ \text { With diode, e.g., triode diode }\end{array}\right.$

DSC Disc shape

DUO Double, e.g., double diode with separate cathodes

DWD $\left\{\begin{array}{l}\text { Duo diode (single cathode) } \\ \text { ( }\end{array}\right.$

With duodiode, e.g., triode duodiode

E Common emitter operation

EL Electrometer tube
ELM Electromagnetic focus or deflection

ELS Electrostatic focus or deflection

F Filamentary cathode

FE Iron cathode - counter tube

FLS Flash tube (photographic)

G Giga $\left(10^{9}\right)$

GAM Gamma radiation

GAN Germanium a11oy, n-type

GAP Germanium a11oy, p-type

GDP Germanium diffused junction, p-type

GE Germanium

GEA Germanium a 11oy junction

GEP Germanium point-contact

GPP Germanium point-contact, p-type

GR $\left\{\begin{array}{l}\text { Green luminescence } \\ \text { Graphite cathode-counter tube }\end{array}\right.$

GS Gas-filled

GSP Germanium surface-barrier, p-type

GTB Gated beam pentode

$\mathrm{H}\left\{\begin{array}{l}\text { Heater type cathode } \\ \text { Hecto }\left(10^{2}\right)\end{array}\right.$

HE Helium gas-filled

HG Mercury vapor-filled 
$\mathrm{HH}$ Mercury-argon-hydrogen gas-filled

HK Hydrogen-krypton gas-filled

HY Hydrogen gas-filled

IC Iconoscope

I Indicator tube

IF Intermediate frequency

IGN Ignitron tube

IM Image orthicon

J Joules

$\mathrm{K} \quad\left\{\begin{array}{l}\mathrm{Kilo}\left(10^{3}\right) \\ \text { Potassium }\end{array}\right.$

\section{KLY KIystron}

KX Krypton-xenon gas-filled

L Linear dynode arrangement

LAM Light

LIT Lighthouse

LO Long persistence screen

$M\left\{\begin{array}{l}\operatorname{Mega}\left(10^{6}\right) \\ \operatorname{Mil1i}\left(10^{-3}\right)\end{array}\right.$

MAG Magnetron

MD Medium persistence screen

MEA Temperature measurement

MG Magnesium cathode

MIX $\left.{ }^{\prime}\right\}$ Mixer

MO Molybdenum cathode
MOD Modulator

$\mathrm{N} \quad$ Nano $\left(10^{-9}\right)$

NA Ne on-argon gas-filled

NE Neon gas-filled

NEH Neon-helium gas-filled

NI Nickel cathode

NK Neon-krypton gas-filled

NSP Nuclear Spectrometry

OD Double beam oscilloscope

OS Oscilloscope

P Pulse operation

PA Power amplifier

PB Purple-blue Iuminescence

PEN Pencil tube

PHC Photoconductive diode

PHM Photomultiplier

PHO Phototube

POW $\left\{\begin{array}{l}\text { Pentode: } \\ \text { With pentode e.g., triode- }\end{array}\right.$ pentode

PTG Pentagrid

REC Rectifier

REG Regulator (voltage)

RD Red luminescence

RF Radio frequency

ROC Rocket tube 
S1-S7 Spectra1 sensitivity of photo surface

S Max. dimension of cathode ray tube face

SM Secondary emission pentode

SAN Silicon a11oy, n-type

SAP Silicon alloy, p-type

SCC Scintillation Counters

SDN Silicon diffused junction, n-type

SH Short persistence screen

SI Silicon

SIA Silicon a11oy junction

SIN Single e.g., single triode

SIP Silicon, point contact

SM Secondary emission pentode

SN Tin cathode-counter tube

SQ Self-quenching type of counter tube

T Thoriated tungsten cathode

TET Tetrode

THM The rmocouple tube

THY Thyratron

TMS Thermistor

TRD With triple diode

TRI $\left\{\begin{array}{l}\text { Triode } \\ \text { With triode e.g., pentode- }\end{array}\right.$ triode
TV Television tube

TWN Twin with separate cathodes, e.g., twin triode

TWT Traveling-wave tube

U $\left\{\begin{array}{l}\text { Micro }\left(10^{-6}\right) \\ \text { U-shaped }\end{array}\right.$

UF U1tra high frequency

V Venetian-blind dynode arrangement

VC Vacuum

VB Violet-blue luminescence

VI Vidicon

VID Video detector

$W\left\{\begin{array}{l}\text { Change of units to watts } \\ \text { Tungsten cathode } \\ \text { Water-cooled }\end{array}\right.$

WG Wave guide coupling

WH White Iuminescence

$\mathrm{X}$ Smallest dimension-rectangular photocathode

XE Xenon gas-filled

YO Ye11ow-orange luminescence

3C Three color screen for television

*) The meaning of these symbols

\#) indicated in the column heading

$\square$ Less than (before digits)

@ Obsolete type 


\begin{tabular}{|c|c|c|c|c|c|}
\hline & & & ROUP I & , NUMERICAL & \\
\hline TYPE NUMBER & KINO & IYPE & $\begin{array}{l}\text { TABLE } \\
\text { NO. }\end{array}$ & SIMILAR TYPES & $\begin{array}{c}\text { COST SPEC. } \\
\text { NO. }\end{array}$ \\
\hline $\begin{array}{r}.24812-18 \\
.3817-35 \\
.3865-135 \\
.425855-12 \\
.62 H 68\end{array}$ & $\begin{array}{l}\text { BAL } \\
\text { BAL } \\
\text { BAL } \\
\text { BAL } \\
\text { PND }\end{array}$ & $\begin{array}{l}\operatorname{SIN} \\
\operatorname{SIN} \\
\operatorname{SIN} \\
\operatorname{SIN} \\
\operatorname{SIN}\end{array}$ & $\begin{array}{l}V I \\
V I \\
V I \\
\text { VI } \\
\text { II }\end{array}$ & & \\
\hline $\begin{array}{c}.6 P 2 B \\
. B 5 B 55-12 \\
F S-A G \\
F S-A O \\
F S-A V\end{array}$ & $\begin{array}{l}\text { PNO } \\
\text { BAL } \\
\text { PHC } \\
\text { PHC } \\
\text { PHC }\end{array}$ & $\begin{array}{l}\operatorname{SIN} \\
\operatorname{SIN}\end{array}$ & $\begin{array}{l}I I \\
V I \\
X V \\
X V \\
X V\end{array}$ & CK505AX & \\
\hline FS-DO & PHC & & $x v$ & & \\
\hline$F S-K G$ & PHC & & $x v$ & & \\
\hline$F S-K O$ & PHC & & $x v$ & & \\
\hline$F S-K V$ & PHC & & $\hat{x v}$ & & \\
\hline$G R-0.8 / 1.6$ & OWO & $\operatorname{SIN}$ & & GR $1-0.25 / 1.5 \square$ & \\
\hline $\begin{array}{r}T G-0.3 / 0.3 \\
T G-0.5 / 1.3 \\
T G I-0.1 / 0.3\end{array}$ & $\begin{array}{l}T R I \\
T E T \\
T R I\end{array}$ & $\begin{array}{l}\text { THY } \\
\text { THY } \\
\text { THY }\end{array}$ & v11 & $\begin{array}{l}T G 1-0.1 / 0.30,884 * \\
T G 1-n .1 / 1.30,2050 *\end{array}$ & \\
\hline $\begin{array}{l}\text { VG0251500 } \\
\text { O1A }\end{array}$ & $\begin{array}{l}\text { OID } \\
\text { REC }\end{array}$ & $\operatorname{SIN}$ & $x 1$ & GR $1-0.25 / 1 . e 0$ & \\
\hline DIB & REC & & $x I$ & & \\
\hline 010 & REC & & $x I$ & & \\
\hline $\begin{array}{l}\text { DIG } \\
\text { Div }\end{array}$ & $\begin{array}{l}\text { REC } \\
\text { REC }\end{array}$ & & $\begin{array}{l}x I \\
\times 1\end{array}$ & & \\
\hline DIYE & REC & & $\hat{x i}$ & & \\
\hline 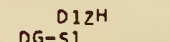 & REC & & $x I$ & & \\
\hline $\begin{array}{c}\text { OG-S1 } \\
D G-T S 1\end{array}$ & MIX & & $\begin{array}{l}x \\
x \\
x\end{array}$ & $02 \mathrm{Gr}$ & \\
\hline OK-II & MIX & & $\hat{x i t I}$ & $0<04$ & \\
\hline $0 K-51$ & MIX & & $x i i i$ & & \\
\hline$O K-V I$ & DET & & $x I 11$ & & \\
\hline$O L-S I$ & $M I X$ & & xiIi & & \\
\hline FEU-1 & PHM & & $x V$ & & \\
\hline $\begin{array}{l}F E U-18 \\
F E U-1 B 1 V\end{array}$ & $\begin{array}{l}\text { PHM } \\
\text { PHM }\end{array}$ & & $\begin{array}{l}x \vee 1 \\
x \vee 1\end{array}$ & & \\
\hline EEU-1B2V & PHM & & $x y z$ & & \\
\hline FEU-IV & PHM & & $x \in I$ & & \\
\hline FEU-15 & PHM & & $x \vee I$ & & \\
\hline$F S-A I$ & PHC & & $x v$ & & \\
\hline$F S-D I$ & PHC & & $x v$ & & \\
\hline FS-KI & PHC & & $x v$ & & \\
\hline GE-1 & TET & $\operatorname{Sin}$ & III & GKE-100 & \\
\hline $\begin{array}{r}G G-1-0.3 / 8 \\
G G 1-0.5 / 5\end{array}$ & $\begin{array}{l}010 \\
010\end{array}$ & $\begin{array}{l}\operatorname{SIN} \\
\operatorname{SIN}\end{array}$ & IV & VG1.5/5000ם & \\
\hline$G G-1-0 \cdot 5 / 20$ & 010 & $\operatorname{SIN}$ & Iv & & \\
\hline $\begin{array}{l}G G-1-1 / 22 \\
G G-1-2 / 5\end{array}$ & $\begin{array}{l}010 \\
010\end{array}$ & $\operatorname{SIN}$ & Iv & & \\
\hline$G G-1-2 / 16$ & DIO & $\operatorname{SIN}$ & iv & & \\
\hline GG-1.5/15 & DIO & $\sin$ & H & GG1-0.5/50 & \\
\hline GK1A & TRI & $\operatorname{SIN}$ & III & & \\
\hline GM1A & TRI & $\operatorname{SIN}$ & 111 & & \\
\hline GMI-1B & TRI & SIN & 111 & & \\
\hline $\begin{array}{r}G R 1-02 / 15 \\
G R-1-0.3 / 8.5\end{array}$ & 010 & $\operatorname{SIN}$ & Iv & & \\
\hline$G R-1-25 / 15$ & OWO & $\sin$ & iv & & \\
\hline $\begin{array}{r}\text { GS-1B } \\
\text { GS-1B }\end{array}$ & TRI & SIN & 111 & (6917 & \\
\hline $\begin{array}{l}\text { GU2H-1 } \\
\quad[-1-70 / 0.8\end{array}$ & $\begin{array}{l}\text { PND } \\
\text { TRI }\end{array}$ & $\begin{array}{l}\text { SIN } \\
\text { THY }\end{array}$ & vil & 6411口 & \\
\hline $1-1-100 / 1.5$ & $T R I$ & THY & vil & & \\
\hline $1-1-140 / 0.8$ & $T R I$ & THY & VII & & \\
\hline $\begin{array}{r}K F-1 \\
K M T-1\end{array}$ & $\begin{array}{l}\text { TET } \\
\text { TMS }\end{array}$ & TWN & $x I x$ & $G U-290,829 B *$ & \\
\hline $\mathrm{K} 2 \mathrm{HI}$ & PNO & $\operatorname{SIN}$ & & G4110 & \\
\hline LOI & PTRI & SiN & & 125350 & \\
\hline$L G-1$ & DWO & $\operatorname{SIN}$ & & $12 \mathrm{KH} 35 \mathrm{O}$ & \\
\hline LI-1 & IC & & vili & & \\
\hline $\begin{array}{l}\text { MMT-1 } \\
\text { MS1 }\end{array}$ & TMS & $\sin$ & $x$ ix & $G M-600$ & \\
\hline DG- & OEC & & $x \times y=$ & & \\
\hline P1B & & & $x$ & & \\
\hline P10 & & & $\hat{x}$ & & \\
\hline
\end{tabular}

\begin{tabular}{|c|c|c|c|c|c|}
\hline \multicolumn{6}{|c|}{ GROUP I, NUMERICAL } \\
\hline TYPE NUMBER & KINO & TYPE & $\begin{array}{l}\text { TABLE } \\
\text { NO. }\end{array}$ & SIMILAR TYPES & $\begin{array}{c}\text { COST SPEC. } \\
\text { HO. }\end{array}$ \\
\hline $\begin{array}{l}\text { PIG } \\
\text { PII } \\
\text { PIV } \\
\text { PIYE } \\
\text { PIZH }\end{array}$ & & & $\begin{array}{l}x \\
x \\
x \\
x \\
x\end{array}$ & & \\
\hline $\begin{array}{l}\text { RB-1 } \\
\text { S1A } \\
\text { S1B } \\
\text { S10 } \\
\text { S1G }\end{array}$ & & & $\begin{array}{l}x \times 11 \\
x \\
x \\
x \\
x\end{array}$ & & \\
\hline $\begin{array}{l}\text { SIV } \\
\text { SIYE } \\
\text { SGIB } \\
\text { SGIP } \\
\text { SGIP-V }\end{array}$ & $\begin{array}{l}010 \\
\text { DIO } \\
\text { REG }\end{array}$ & $\begin{array}{l}\operatorname{SIN} \\
\operatorname{SIN}\end{array}$ & $\begin{array}{l}x \\
x \\
v \\
v\end{array}$ & $\begin{array}{l}\text { OA2* } \\
\text { OA2* }\end{array}$ & \\
\hline $\begin{array}{l}S G I P-Y E \\
S I-1 B G \\
S I-1 G \\
T-1 B \\
T G 1 B\end{array}$ & $\begin{array}{l}\text { REG } \\
\text { CDU } \\
\text { CDU } \\
\text { TRI } \\
\text { TRI }\end{array}$ & $\begin{array}{l}\text { THY } \\
\text { THY }\end{array}$ & $\begin{array}{l}v \\
x \times I \\
x \times I \\
v I I\end{array}$ & $T G-18 \square$ & \\
\hline $\begin{array}{l}T G 18-V \\
T G 1-00 / 05 \\
T G 1-01 / 03 \\
T G 1-01 / 13 \\
T G 1-05 / 12\end{array}$ & $\begin{array}{l}\text { TRI } \\
\text { TET } \\
\text { TRI } \\
\text { TET } \\
\text { TRI }\end{array}$ & $\begin{array}{l}\text { THY } \\
\text { THY } \\
\text { THY } \\
\text { THY } \\
\text { THY }\end{array}$ & $\begin{array}{l}\text { VII } \\
\text { VII } \\
\text { VII } \\
\text { vII } \\
\text { VII }\end{array}$ & $\begin{array}{l}884 * \\
2050 *\end{array}$ & $7843-55$ \\
\hline $\begin{array}{l}T G 1-1.0 / 0 \\
T G 1-1.5 / 2 \\
T G 1-1.6 / 1 \\
T G 1-2.5 / 3 \\
T G 1-2.5 / 4\end{array}$ & $\begin{array}{l}\text { TET } \\
\text { TRI } \\
\text { TRI } \\
\text { TRI } \\
\text { TRI }\end{array}$ & $\begin{array}{l}\text { THY } \\
\text { THY } \\
\text { THY } \\
\text { THY } \\
\text { THY }\end{array}$ & $\begin{array}{l}\text { VII } \\
\text { VII } \\
\text { VII } \\
\text { VII } \\
\text { VII }\end{array}$ & $\begin{array}{l}T G 1-2,5 / 4 e a \\
T G 8 / 3, T G 1-2,5 / 300\end{array}$ & $7952-56$ \\
\hline $\begin{array}{r}T G 1-2.5 / 10 \\
T G 1-3.2 / 1 \\
T G 1-6.4 / 1 \\
T G 1-5 / 3 \\
T G 1-12.5 / 1.3\end{array}$ & $\begin{array}{l}\text { TRI } \\
\text { TRI } \\
\text { TRI } \\
\text { TRI } \\
\text { TRI }\end{array}$ & $\begin{array}{l}\text { THY } \\
\text { THY } \\
\text { THY } \\
\text { THY } \\
\text { THY }\end{array}$ & $\begin{array}{l}\text { VII } \\
\text { VII } \\
\text { VII } \\
\text { VII } \\
\text { VII }\end{array}$ & & \\
\hline $\begin{array}{l}T G 1-25 / 10 \\
T G 1-125 / 1 \\
T G I-n, 1 / 0.3 \\
T G I-1 B \\
T G I-1-3 / 1\end{array}$ & $\begin{array}{l}\text { TRI } \\
\text { TRI } \\
\text { TRI } \\
\text { TRI } \\
\text { TET }\end{array}$ & $\begin{array}{l}\text { THY } \\
\text { THY } \\
\text { THY } \\
\text { THY } \\
\text { THY }\end{array}$ & $\begin{array}{l}\text { VII } \\
\text { VII } \\
\text { VII } \\
\text { VII } \\
\text { VII }\end{array}$ & & \\
\hline $\begin{array}{l}T G I-1-10 / 1 \\
T G I-1-35 / 3 \\
T G I-1-50 / 5 \\
T G I-1-90 / 8 \\
T G I-1-130 / 8\end{array}$ & $\begin{array}{l}\text { TRI } \\
\text { TRI } \\
\text { TRI } \\
\text { TRI }\end{array}$ & $\begin{array}{l}\text { THY } \\
\text { THY } \\
\text { THY } \\
\text { THY } \\
\text { THY }\end{array}$ & $\begin{array}{l}\text { VII } \\
\text { VII } \\
\text { VII } \\
\text { VII } \\
\text { VII }\end{array}$ & MTI-400 & \\
\hline $\begin{array}{r}T G I-1-130 / 10 \\
T G I-1-325 / 16 \\
T G I-1-400 / 3 \\
T G I-1-400 / 16 \\
T G I-1-700 / 25\end{array}$ & $\begin{array}{l}\text { TRI } \\
\text { TRI } \\
\text { TRI } \\
\text { TRI } \\
\text { TRI }\end{array}$ & $\begin{array}{l}\text { THY } \\
\text { THY } \\
\text { THY } \\
\text { THY } \\
\text { THY }\end{array}$ & $\begin{array}{l}\text { VII } \\
\text { VII } \\
\text { VII } \\
\text { VII } \\
\text { VII }\end{array}$ & MTI-5口, TGI-325/16ם & \\
\hline $\begin{array}{l}\text { TKH1 } \\
T M-1 \\
T O-1\end{array}$ & $\begin{array}{l}\text { TRI } \\
\text { TRI } \\
\text { PNO }\end{array}$ & $\begin{array}{l}\text { THY } \\
\text { SIN } \\
\text { SIN }\end{array}$ & VII & $\begin{array}{l}6550 \mathrm{O}, 2 \mathrm{C}_{40 *} \\
102 \mathrm{H1} 2 \mathrm{Sa}\end{array}$ & \\
\hline $\begin{array}{l}T R 1-5 / 2 \\
T R 1-6 / 15\end{array}$ & $\begin{array}{l}\text { TRI } \\
\text { TRI }\end{array}$ & $\begin{array}{l}\text { THY } \\
\text { THY }\end{array}$ & $\begin{array}{l}\text { VII } \\
\text { VII }\end{array}$ & $V T-3$ & $\begin{array}{l}7954-56 \\
7955-56\end{array}$ \\
\hline $\begin{array}{r}T R 1-15 / 15 \\
T R 1-40 / 15 \\
T R 1-85 / 15 \\
T R 1-13015 \\
T S G=1\end{array}$ & $\begin{array}{l}\text { TRI } \\
\text { TRI } \\
\text { TRI } \\
\text { TRI } \\
\text { PHO }\end{array}$ & $\begin{array}{l}\text { THY } \\
\text { THY } \\
\text { THY } \\
\text { THY }\end{array}$ & $\begin{array}{l}\text { VII } \\
\text { VII } \\
\text { VII } \\
\text { VII } \\
\text { XV }\end{array}$ & & $7956-56$ \\
\hline $\begin{array}{l}\text { TSV-1 } \\
\text { TVB-1 } \\
V 1-00313 \\
V 1-02 / 20 \\
V 1=03 / 13\end{array}$ & $\begin{array}{l}\text { PHO } \\
\text { THM } \\
\text { DIO } \\
\text { DIO } \\
\text { DID }\end{array}$ & $\begin{array}{l}\operatorname{SIN} \\
\operatorname{SIN} \\
\operatorname{SIN}\end{array}$ & $\begin{array}{l}\text { XV } \\
\text { XVIII } \\
\text { IV } \\
\text { IV } \\
\text { IV }\end{array}$ & V13/3nם & \\
\hline $\begin{array}{l}v 1-05 / 70 \\
v 1-06 / 30 \\
v 1-1 / 2.5 \\
v 1-1 / 30 \\
v 1-1 / 40\end{array}$ & $\begin{array}{l}010 \\
010 \\
010 \\
010 \\
010\end{array}$ & $\begin{array}{l}\operatorname{Sin} \\
\operatorname{Sin} \\
\operatorname{Sin} \\
\operatorname{Sin} \\
\operatorname{Sin}\end{array}$ & $\begin{array}{l}\text { Iv } \\
\text { Iv } \\
\text { Iv } \\
\text { iv } \\
\text { iv }\end{array}$ & & \\
\hline $\begin{array}{l}v_{1}-2 / 40 \\
v_{1}-3 / 16\end{array}$ & $\begin{array}{l}010 \\
010\end{array}$ & $\begin{array}{l}\operatorname{SIN} \\
\operatorname{SIN}\end{array}$ & $\begin{array}{l}\text { IV } \\
\text { IV }\end{array}$ & & \\
\hline
\end{tabular}




\begin{tabular}{|c|c|c|c|c|c|}
\hline \multicolumn{6}{|c|}{ GROUP I. NUMERICAL } \\
\hline TYPE NUMBER & sino & IYPE & $\begin{array}{l}\text { TA8LE } \\
\text { No. }\end{array}$ & SINILAR TYPES & $\begin{array}{c}\text { COST SPEC. } \\
\text { No. }\end{array}$ \\
\hline V1 $-3 / 70$ & 010 & $\operatorname{SIN}$ & Iv & & \\
\hline$V 1-4 / 40$ & DIO & $\operatorname{SIN}$ & iv & & \\
\hline$v 1-15 / 35$ & 010 & SIN & iv & & \\
\hline VOI & DIO & $S$ IN & & $v 1-1 / 40 a$ & \\
\hline VDI -10 & 010 & $\operatorname{SIN}$ & & $V I-1-100 / 500$ & \\
\hline VG $1 / 8500$ & 010 & SIN & IV & & \\
\hline VGI.3/5000 & 010 & SIN & IV & $G G 2-D \cdot 5 / 50$ & \\
\hline$V I-1-5 / 20$ & 010 & SIN & Iv & & \\
\hline V $1-1-5 / 30$ & 010 & 5 IN & IV & & \\
\hline$V I-1-18 / 32$ & DIO & SIN & IV & & \\
\hline$V I-1-27 / 35$ & 010 & $\operatorname{SIN}$ & iv & & \\
\hline$V I-1-30 / 25$ & 010 & $\operatorname{SIN}$ & Iv & & \\
\hline$v 1-1-7 n / 32$ & 010 & SIN & IV & & \\
\hline$V I-1-100 / 50$ & 010 & $5 I N$ & Iv & & \\
\hline vo-1 & 010 & $\operatorname{SIN}$ & Iv & & \\
\hline VSTS- $1 \quad F-3$ & PHD & & $x v$ & & \\
\hline$V T-1$ & $T R !$ & THY & & $T G-2.5 / 50$ & \\
\hline IAIP & PTG & SIN & II & 185*, DK91* & \\
\hline $\begin{array}{l}1 A 2 P \\
1 B 1 P\end{array}$ & $\begin{array}{l}\text { PTG } \\
\text { PND }\end{array}$ & $\begin{array}{l}\text { SIN } \\
\text { DID }\end{array}$ & $\begin{array}{ll}11 \\
11\end{array}$ & $\begin{array}{l}\text { DK96*, 1R5* } \\
155 *, \text { DAF } 91 \text { * }\end{array}$ & $8006-56$ \\
\hline $182 P$ & PNO & 010 & 11 & DAF $96 *$ * 155* & \\
\hline $185-9$ & $B A L$ & $\sin$ & VI & & \\
\hline $1810-17$ & $B A L$ & $\operatorname{SIN}$ & VI & & \\
\hline IEIP & TET & $\sin$ & 11 & & \\
\hline $1 E 3 P$ & •TRI & $\sin$ & it & $E M-4 D$ & \\
\hline $112 \mathrm{P}$ & PND & $T R I$ & 11 & & \\
\hline $1 K 1 P$ & PND & SIN & 11 & $1 T 4 *$, DF $91 *$ & $7707-55$ \\
\hline $1 K 2 P$ & PND & $\operatorname{SIN}$ & 11 & DF 96 * , 1T4* & \\
\hline 1N1 & -TRI & THN & & 1 N3S & \\
\hline $1 \times 35$ & TRI & $T W N$ & II & $1 N 1 E, \quad 1 G 6-G T^{*}$ & \\
\hline $1 P 2 B$ & PND & $S ! N$ & 11 & CK5D7AX & \\
\hline 1 P 38 & PND & $\operatorname{SIN}$ & $1 !$ & & \\
\hline $1 P \backsim 8$ & PNO & $\sin$ & $!$ & & \\
\hline $1 P 24 B$ & PND & $\operatorname{SIN}$ & II & & \\
\hline $1512 P$ & TRI & SIN & II & DC96" & \\
\hline $1 T 51$ & D ID & SIN & & $1 T 5150,1$ VDIa & \\
\hline $1 T 515$ & DID & SIN & II & 1T510, 1VOID & \\
\hline 1 TS7S & DID & SIN & 11 & OY $30 *, 183 / 8016 *$ & $B 359-57$ \\
\hline 1TS11P & 010 & $\operatorname{Sin}$ & 11 & & \\
\hline $1 V_{3} / 8016$ & D IO & S IN & & $1 T S 750,183 / 8016 *$ & \\
\hline 1 VOI & QDID & SIN & & $1 T 51,1 T S 150$ & \\
\hline $1 \mathrm{VD} 2$ & -010 & SIN & & 1TS7SA, 1B3/BD16* & \\
\hline $12 \mathrm{H}_{2}$ & $\triangle P N D$ & $\operatorname{SIN}$ & & $12 \mathrm{H} 2 \mathrm{Ma}$ & \\
\hline $12 \mathrm{H}_{2} \mathrm{M}$ & PND & $\operatorname{SIN}$ & II & $12 \mathrm{H} 20$ & \\
\hline $12 \mathrm{H} 17 \mathrm{~B}$ & PND & SIN & II & & \\
\hline $12 \mathrm{H1} 88$ & OND & $\operatorname{SIN}$ & II & & \\
\hline $12 H_{2} 48$ & PND & SIN & II & & \\
\hline $12 \mathrm{H}_{2} 98$ & PND & SIN & I! & & \\
\hline D2A & $\bullet R E C$ & & $x I$ & DG-TS9:a & \\
\hline $\mathrm{D} 2 \mathrm{~B}$ & -REC & & $x I$ & DG-TS10॰ & \\
\hline $\begin{array}{l}020 \\
02 \mathrm{G}\end{array}$ & REC & & $x !$ & $\begin{array}{l}O G=T S 290 \\
O G-T S 190\end{array}$ & \\
\hline $\begin{array}{l}02 \mathrm{G} \\
02 \mathrm{I}\end{array}$ & $\begin{array}{l}\text { REC } \\
\text { REC }\end{array}$ & & $\begin{array}{l}x I \\
x I\end{array}$ & $D G-T S 1$ ค & \\
\hline $\begin{array}{l}02 \mathrm{I} \\
02 \mathrm{~K}\end{array}$ & $\begin{array}{l}\text { REC } \\
\text { REC }\end{array}$ & & $\hat{X I}$ & $D G=T S 6 P \square$ & \\
\hline $02 M$ & REC & & $x 1$ & OG-TS7@ם & \\
\hline$D 2 N$ & REC & & XI & DG-TS1 sed & \\
\hline $\mathrm{D} 2 \mathrm{P}$ & REC & & $x I$ & $D G-T 516 \circ 0$ & \\
\hline $\mathrm{D} 2 \mathrm{R}$ & REC & & $x I$ & & \\
\hline D2V & REC & & $x I$ & $\begin{array}{l}D G-T S B= \\
D G-T S 490\end{array}$ & \\
\hline OZYE & -REC & & $x I$ & DG-TS4P口 & \\
\hline $\begin{array}{l}D 2 Z H \\
D G-52\end{array}$ & $\begin{array}{l}\text { - REC } \\
\text { MIX }\end{array}$ & & $\begin{array}{l}x I \\
x I I I\end{array}$ & DG-TS5^ם & \\
\hline $\begin{array}{l}D G-52 \\
D G-T S 2\end{array}$ & $\begin{array}{l}\text { MIX } \\
\text { REC }\end{array}$ & & $\begin{array}{l}x ! 11 \\
x !\end{array}$ & 0200 & \\
\hline$D I-2-10$ & $9 D I D$ & $\operatorname{SIN}$ & & 20150 & \\
\hline$D K-I 2$ & MIX & & $x 111$ & & \\
\hline$O K-\$ 2$ & $M[x$ & & $x I I I$ & & \\
\hline$O K-V 2$ & OET & & $x I I I$ & & \\
\hline$O L-52$ & $M I x$ & & $x \| I$ & & \\
\hline $\begin{array}{l}\text { DSH2-1D } \\
\text { FEU-2 }\end{array}$ & $\begin{array}{l}\text { DIO } \\
\text { PHM }\end{array}$ & SIN & $x v$ & 20250 & \\
\hline$=\varepsilon U-2 B$ & PHM & & $x \vee I$ & & \\
\hline$F E U-2 B I V$ & PHM & & $x \vee I$ & & \\
\hline
\end{tabular}

\begin{tabular}{|c|c|c|c|c|c|}
\hline \multicolumn{6}{|c|}{ GROUP I. NUMERICAL } \\
\hline TYPE NUMBER & kino & IYPE & $\begin{array}{l}\text { WABLE } \\
\text { No. }\end{array}$ & SIMILAR TYPES & $\begin{array}{l}\text { COST SPEC. } \\
\text { MO. }\end{array}$ \\
\hline$F E U-2 M$ & PHM & & $x \vee I$ & & \\
\hline$F E U-2 V$ & PHM & & xVI & & \\
\hline $\mathrm{FS}-\mathrm{B2}$ & PHC & & $x y$ & & \\
\hline$F S-K 2$ & PHC & & $x v$ & & \\
\hline$F S-2 A$ & PHC & ( & $x v$ & & \\
\hline $\begin{array}{c}G E-2 \\
G M I-2 B\end{array}$ & $\begin{array}{l}\text { TET } \\
\text { TET }\end{array}$ & $\begin{array}{l}\operatorname{SIN} \\
\operatorname{SIN}\end{array}$ & III & GKE-150 & \\
\hline$G s-2 B$ & TRI & $\operatorname{SIN}$ & ii & & \\
\hline $\mathrm{GU} Z \mathrm{H}-2$ & $B E A$ & SIN & & G8070, $807 *$ & \\
\hline $\mathrm{G} 7 \mathrm{H} 2$ & PND & SIN & & G4130 & \\
\hline $1-2-50 / 1.5$ & $T R !$ & THY & VII & & \\
\hline$K F-2$ & $B E A$ & TWN & & $G U-32 D, 832-A *$ & \\
\hline $\mathrm{Ks}-2$ & $T R I$ & $\operatorname{SIN}$ & & $G U-4 a$ & \\
\hline $\begin{array}{l}\mathrm{K} 2 \mathrm{H}-2 \\
\mathrm{MTI}-2\end{array}$ & $\begin{array}{l}\text { BEA } \\
\text { TRI }\end{array}$ & $\begin{array}{l}\text { SIN } \\
\text { THY }\end{array}$ & VII & $\begin{array}{l}G-8070,807 * \\
T G I-2 \cap D \square\end{array}$ & \\
\hline$D G-2$ & OEC & & $x \times I I I$ & & \\
\hline $\begin{array}{l}\text { P2A } \\
\text { P2B }\end{array}$ & & & $\begin{array}{l}x \\
x\end{array}$ & & \\
\hline PT-2 & $T R I$ & THY & & $T G-213 \theta$ & \\
\hline$R-2$ & & & $x \times I I$ & & \\
\hline$R B-2$ & & & $x \times I I$ & & \\
\hline $52 A$ & & & $x$ & & \\
\hline S2B & & & $x$ & & \\
\hline $52 \mathrm{G}$ & & & $x$ & & \\
\hline $52 \mathrm{~V}$ & & & $x$ & & \\
\hline$S G 2 P$ & D10 & SIN & $v$ & DB2* & \\
\hline $5 G 2 S$ & 010 & SIN & v & OA 3* & \\
\hline $51-2 B$ & cou & & $x \times I$ & & \\
\hline$S I-2 B G$ & cou & & $x \times 1$ & 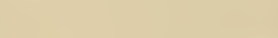 & \\
\hline ST 25 & $B A L$ & TWN & VI & & \\
\hline STS-2 & $\mathrm{cou}$ & & $x \times I$ & & \\
\hline ST SV $-2 A \quad F-2$ & PHO & & $x V$ & & \\
\hline $\begin{array}{r}T G 2-D 1 / 01 \\
T G 2-0.5 / 12\end{array}$ & $T R I$ & THY & VII & & \\
\hline $\begin{array}{l}T G 2-0.5 / 12 \\
T G-2 \cdot 5 / 5\end{array}$ & $\begin{array}{l}T R I \\
T R I\end{array}$ & THY & VII & $V T-1$ & \\
\hline$T G I-2-260 / 12$ & $T R I$ & THY & VII & & \\
\hline$T G I-2-32516$ & $T R I$ & THY & vil & & \\
\hline $\begin{array}{l}\text { TGI }=2-40035 \\
T K H=?\end{array}$ & TRI & THY & VII & & \\
\hline $\begin{array}{r}\text { TKH-2 } \\
T D-2\end{array}$ & $\begin{array}{l}\text { TRI } \\
\text { PND }\end{array}$ & $\begin{array}{l}\text { THY } \\
\text { SIN }\end{array}$ & VII & $10 P 125 \square$ & \\
\hline$T V-2$ & THM & & XVIII & & \\
\hline$T V B-2$ & THM & & $x \vee I I I$ & & \\
\hline VD2 & 010 & SIN & & $v 1-2 / 400$ & \\
\hline $\begin{array}{l}v I-2-27 / 35 \\
V I-2-70 / 32\end{array}$ & $\begin{array}{l}\text { DIO } \\
\text { DIO }\end{array}$ & $\begin{array}{l}\operatorname{SIN} \\
\operatorname{SIN}\end{array}$ & $\begin{array}{l}\text { IV } \\
\text { IV }\end{array}$ & & \\
\hline$V I-2-100 / 50$ & 010 & $\operatorname{SIN}$ & Iv & & \\
\hline $2 A 1$ & PTG & $\operatorname{SIN}$ & iI & $502420,2 A 1 M$ & \\
\hline 2AIM & ePTG & $\operatorname{SIN}$ & & $2 A 10$ & \\
\hline 243 & TRI & $\operatorname{SIN}$ & & $25450,2 A 3 *$ & \\
\hline $201 \mathrm{~L}$ & DWD & SIN & II & & \\
\hline $201 \mathrm{~s}$ & DID & $\operatorname{SIN}$ & II & $01-2-100$ & \\
\hline 2025 & DID & $\operatorname{SIN}$ & II & $\mathrm{DSH} 2-10 \mathrm{OO}$ & \\
\hline $203 \mathrm{~B}$ & 010 & $\operatorname{SIN}$ & II & & \\
\hline $\begin{array}{l}2035 \\
2075\end{array}$ & $\begin{array}{l}\text { DID } \\
\text { DID }\end{array}$ & $\begin{array}{l}\operatorname{SIN} \\
\operatorname{SIN}\end{array}$ & It & & \\
\hline 2095 & DID & $\operatorname{SIN}$ & II & & \\
\hline 2021 & TET & THY & & TG 3-n.1/1.30, 2021* & \\
\hline $2 \mathrm{EI}$ & TET & SIN & II & & \\
\hline $\begin{array}{l}2 E 2 \\
2 E 2 P\end{array}$ & OTET & $\operatorname{SIN}$ & II & U81550 & \\
\hline & TET & TWN & II & & \\
\hline $\begin{array}{l}2 J 55 \\
2 K 1\end{array}$ & $\begin{array}{l}\text { MAG } \\
\text { PAND }\end{array}$ & & Ix & 2K1MD & \\
\hline $2 K 1 M$ & PND & SIN & II & $2 \mathrm{~K} 1 \mathrm{e}, 58241 \mathrm{e}, 5 \mathrm{~S}_{2410}$ & \\
\hline $2 \times 2$ & PND & SIN & & $2 \mathrm{~K} 2 \mathrm{MP}$ & \\
\hline $2 \mathrm{~K} 2 \mathrm{M}$ & PND & SIN & II & $2 \times 2 \circ$ & \\
\hline $2 \mathrm{KH} 1$ & DW 0 & $\operatorname{SIN}$ & & 2KHILG & \\
\hline $2 \mathrm{KHIL}$ & DWO & $\sin$ & II & $2 \mathrm{KHI}$ & \\
\hline $2 \mathrm{KH} 2$ & CDIO & SIN & & 2VDBAG, $2 T S 2 S \sigma, 2 \times 2 *$ & \\
\hline $2 \mathrm{NI}$ & TRI & DUO & 11 & $58243,50243,2 N 1 M 0$ & \\
\hline 2N1M & ETRI & DUD & & $2 N_{10}, 582430,502430$ & \\
\hline $\begin{array}{l}2 P 1 \\
2 P 1 M\end{array}$ & $\begin{array}{r}\text { BEA } \\
\text { CBEA }\end{array}$ & $\begin{array}{l}\operatorname{SIN} \\
\operatorname{SIN}\end{array}$ & II & $\begin{array}{l}582440,502440 \\
2 P 1 P a\end{array}$ & \\
\hline
\end{tabular}




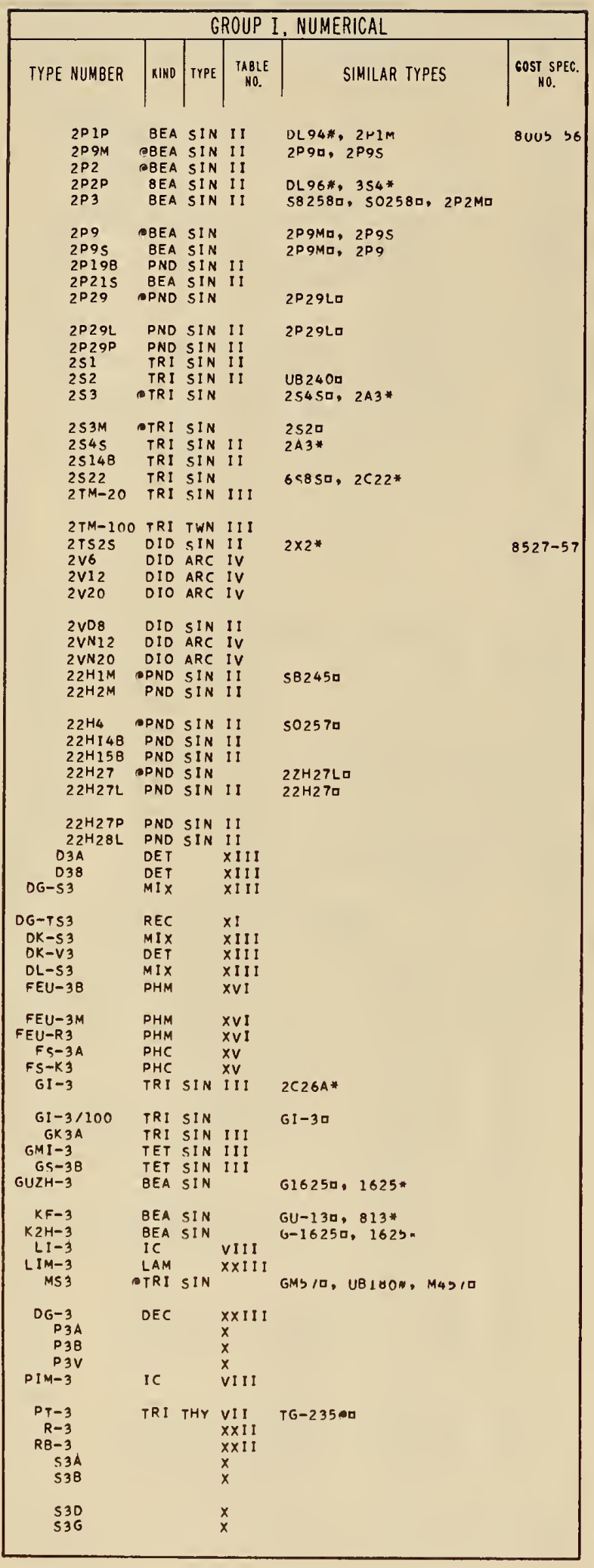

\begin{tabular}{|c|c|c|c|c|c|}
\hline \multicolumn{6}{|c|}{ GROUP I, NUMERICAL } \\
\hline TYPE NUMBER & KINO & TYPE & $\begin{array}{l}\text { TABLE } \\
\text { NO. }\end{array}$ & SIMILAR TYPES & $\begin{array}{l}\text { COST SPEC. } \\
\text { Ho. }\end{array}$ \\
\hline $53 \mathrm{~V}$ & & & $x$ & & \\
\hline S3YE & & & $x$ & & \\
\hline $\begin{array}{l}\text { SG3P } \\
\text { SG3S }\end{array}$ & REG & SIN & $\begin{array}{l}v \\
v\end{array}$ & & \\
\hline $5 I-38$ & cou & $51 \mathrm{~N}$ & $x \times I$ & $0<3 *$ & \\
\hline ST $3 P$ & 010 & $\operatorname{SIN}$ & VI & & \\
\hline $\begin{array}{l}\text { STS-3 } \\
\text { STSV }-3\end{array}$ & $\begin{array}{l}\text { CDU } \\
\text { PHD }\end{array}$ & & $\begin{array}{l}x \times I \\
x y\end{array}$ & & \\
\hline $\begin{array}{r}T G_{3}-01 / 13 \\
\end{array}$ & TET & THY & $\begin{array}{l}x V \\
V I I\end{array}$ & $2021 *$ & \\
\hline$T 63-2.5 / 10$ & TRI & THY & VII & & \\
\hline $\begin{array}{l}\text { TKH3B } \\
\text { TD }-3\end{array}$ & $\begin{array}{l}\text { TET } \\
\text { PND }\end{array}$ & THY & VII & & \\
\hline TSG-3 & PHD & Sin & $x y$ & $72 \mathrm{H} 125 \mathrm{~B}$ & \\
\hline TSV-3 & PHO & & & & \\
\hline TV8-3 & THM & & $x \vee 111$ & & \\
\hline $\begin{array}{l}\text { VDI-3D } \\
\text { VT }-3\end{array}$ & DID & SIN & & $V I-1-30 / 25 \square$ & \\
\hline $3 A 45$ & $\begin{array}{l}\text { TRI } \\
\text { PND }\end{array}$ & $\begin{array}{l}\text { THY } \\
\text { SIN }\end{array}$ & II & TR1-5/20口 & \\
\hline 3845 & 8EA & $\operatorname{SIN}$ & 11 & & \\
\hline $3 E 29$ & PBEA & TWN & & GI-30D, 3E29* & \\
\hline $3 J 21$ & MAG & & Ix & & \\
\hline $\begin{array}{l}3 L_{1} 1-1 \\
351\end{array}$ & TR I & SIN & VIII $_{\text {II }}$ & $T D-1410$ & \\
\hline 352 & TRI & SIN & iI & $T D-142 \square$ & \\
\hline 359 & CTRI & $\operatorname{Sin}$ & II & & \\
\hline $3 \mathrm{TS} 16 \mathrm{~S}$ & D10 & & II & & \\
\hline $\begin{array}{l}3 T S 18 P \\
3 \vee 30\end{array}$ & $\begin{array}{l}\text { OID } \\
010\end{array}$ & $\begin{array}{l}\text { SIN } \\
\text { ARC }\end{array}$ & Iv & & \\
\hline 3 VN 30 & DIO & $A R C$ & Iv & & \\
\hline 3 VN60 & DID & ARC & IV & & \\
\hline $\begin{array}{l}\text { 3VN } 100 \\
3 \text { VP }\end{array}$ & DID & ARC & IV & & \\
\hline $\mathrm{DG}-54^{3 V^{2} 1}$ & MIX & & XIII & $8 L D 290,3 B P 1 A^{*}$ & \\
\hline DG-TS4 & REC & & $x I$ & D. YEEA & \\
\hline DK-S4 & MIX & & XIII & & \\
\hline DK $-\sqrt{ } 4$ & DET & & $x I 11$ & & \\
\hline $\begin{array}{r}D L-54 \\
E M-4\end{array}$ & $\begin{array}{l}\text { MIX } \\
\text { TRI }\end{array}$ & $\sin$ & $x+11$ & IE 3P口 & \\
\hline FS-A4 & PHC & & $x y$ & & \\
\hline FS-K4 & PHC & & $x V$ & & \\
\hline $\begin{aligned} G i-4 A \\
G K V-4\end{aligned}$ & TRI & $\operatorname{SIN}$ & 111 & & \\
\hline $\begin{array}{l}\text { GKV }-4 \\
G M I-48\end{array}$ & $\begin{array}{l}\text { TRI } \\
\text { TET }\end{array}$ & $\begin{array}{l}\operatorname{Sin} \\
\sin \end{array}$ & $I I I$ & GU-4D & \\
\hline $\begin{array}{l}G s-4 \\
G S-4\end{array}$ & TRI & $\sin$ & $\operatorname{lil}_{x \times I}$ & & \\
\hline & $\cos$ & . & $x \times 1$ & & \\
\hline $\begin{array}{r}G S-4 B \\
G S 4 D\end{array}$ & TRI & $\sin$ & & G431A口 & \\
\hline $\begin{array}{l}\text { G54D } \\
\text { GU4 }\end{array}$ & $\begin{array}{l}T R I \\
T R I\end{array}$ & $\begin{array}{l}\operatorname{Sin} \\
\operatorname{Sin}\end{array}$ & III & & \\
\hline GU4A & $T R I$ & $\sin$ & iii & & \\
\hline KMT-4 & TMS & & $x i x$ & & \\
\hline $\begin{array}{r}K S-4 \\
\text { LIM-4 }\end{array}$ & TRI & $\operatorname{SIN}$ & & $G U-150 \square$ & \\
\hline $\begin{array}{r}\operatorname{LIM}-4 \\
\operatorname{LP}-4\end{array}$ & $\begin{array}{l}\text { LAM } \\
\text { COM }\end{array}$ & $A C D$ & $\begin{array}{l}x \times 111 \\
\text { V11 }\end{array}$ & & \\
\hline MMT -4 & TMS & & $x I x$ & & \\
\hline $\mathrm{MS}-4$ & $\mathrm{COU}$ & & $x \times I$ & & \\
\hline MSTR-4 & CDU & & $x \times 1$ & & \\
\hline $\begin{array}{c}M T I-4 \\
D G-5\end{array}$ & $\begin{array}{l}\text { TRI } \\
\text { DEC }\end{array}$ & THY & $\begin{array}{l}\text { vil } \\
x \times 111\end{array}$ & $T G I-1-90 / 8 \square$ & \\
\hline $\begin{array}{l}P_{4} \\
P_{4} \mathrm{~A}\end{array}$ & & & $\begin{array}{l}x \\
x\end{array}$ & 2 N68* & \\
\hline P48 & & & $x$ & & \\
\hline P4D & & & $x$ & & \\
\hline $\begin{array}{l}P 4 G \\
P 4 L\end{array}$ & & & $\begin{array}{l}x \\
x\end{array}$ & & \\
\hline $\begin{array}{l}P_{4} L \\
P_{4} V\end{array}$ & & & $\begin{array}{l}x \\
x\end{array}$ & & \\
\hline $\begin{array}{l}P I M-4 \\
R-4\end{array}$ & IC & & VIII & & \\
\hline $\begin{array}{l}R-4 \\
S 4 A\end{array}$ & & & $\begin{array}{l}x \times 11 \\
x\end{array}$ & & \\
\hline $54 B$ & & & $\hat{x}$ & & \\
\hline $54 \mathrm{G}$ & & & $x$ & & \\
\hline $\begin{array}{l}54 V \\
5 G 45\end{array}$ & 010 & $\sin$ & $\begin{array}{l}x \\
v\end{array}$ & DD3* & \\
\hline
\end{tabular}




\begin{tabular}{|c|c|c|c|c|c|c|}
\hline \multicolumn{7}{|c|}{ GROUP I, NUMERICAL } \\
\hline TYPE NUYBER & 110 & Irfe & $\begin{array}{c}\text { Thote } \\
\text { no. }\end{array}$ & SINILAR TYPES & & $\begin{array}{c}\text { COSI SPEC. } \\
10 .\end{array}$ \\
\hline$S I-\Delta G$ & $\mathrm{COU}$ & & $x \times 1$ & & $\vdots$ & \\
\hline STSV -4 & PHD & & $x v$ & & & \\
\hline TGI-4 & TRI & THY & & $T G I-1-130 / 100$ & & \\
\hline$T K H-4 B$ & TET & THY & VII & & & \\
\hline ro-4 & PND & $\sin$ & & $7 P 1250$ & & \\
\hline TSG -4 & PHD & & $x V$ & & & \\
\hline TSV-A & PHO & & XV & & & \\
\hline TV-A & THM & & $X \vee I I I$ & & & \\
\hline$T \vee B-4$ & THM & & $x \vee I I I$ & & & \\
\hline VOI -40 & DID & $\sin$ & & $V I-1-70 / 32 a$ & & \\
\hline Vs $=\Delta$ & CDU & & $x \times 1$ & & & \\
\hline$\triangle 02$ & 010 & $\operatorname{stn}$ & & $4 T 5650$ & & \\
\hline $\begin{array}{l}\triangle D S S \\
\text { CEI }\end{array}$ & $\begin{array}{l}\text { D ID } \\
\text {-TET T }\end{array}$ & $\begin{array}{l}\text { SIN } \\
\text { SIN }\end{array}$ & $\begin{array}{l}\text { II } \\
\text { II }\end{array}$ & & & \\
\hline LE2 & ATET & $\operatorname{Sin}$ & ii & & & \\
\hline$\Delta \varepsilon^{3}$ & -T ET & $\operatorname{SIN}$ & I! & & & \\
\hline 4565 & $\begin{array}{l}B E A \\
M A G\end{array}$ & $\operatorname{SIN}$ & II & & & \\
\hline $\begin{array}{l}\text { J26-30 } \\
445\end{array}$ & $\begin{array}{l}M A G \\
M A G\end{array}$ & & $\begin{array}{l}\text { Ix } \\
\text { Ix }\end{array}$ & & & \\
\hline $4 J 50$ & MAG & & $\hat{x}$ & & & \\
\hline 4 N1 & TRI & DUD & II & 582590,502390 & & \\
\hline$\triangle P 1$ & -PND & $\operatorname{SIN}$ & It & & & \\
\hline $4 P 1 L$ & PND & $\sin$ & I! & & & \\
\hline $\begin{array}{l}\text { A } 105 \\
\text { Q } 51\end{array}$ & $\begin{array}{l}\text { PND } \\
\text { TR! }\end{array}$ & $\begin{array}{l}\text { SIN } \\
\text { SIN }\end{array}$ & $\begin{array}{l}\text { II } \\
\text { II }\end{array}$ & 481070 & & \\
\hline & & & & & & \\
\hline 452 & $T R I$ & $\operatorname{SIN}$ & II & 481100 & & \\
\hline 453 & -TRI & $\operatorname{Sin}$ & It & & & \\
\hline 4535 & TRI & SIN & II & & & \\
\hline 454 & TRI & SIN & II & & & \\
\hline $45 s$ & TRI & $\sin$ & It & $50-1850$ & & \\
\hline $4 T S I M$ & 9010 & SIN & & $4 T 5650$ & & \\
\hline $4 T 565$ & 010 & SIN & II & & & \\
\hline $\begin{array}{l}\triangle T S I \$ 5 \\
\text { VDI }\end{array}$ & $\begin{array}{l}010 \\
010\end{array}$ & $\begin{array}{l}\text { SIN } \\
\text { SIN }\end{array}$ & $\begin{array}{l}\text { II } \\
\text { II }\end{array}$ & & & \\
\hline$\triangle V K H I$ & - D10 & SWN & II & vo- 18800 & & \\
\hline$\triangle \mathrm{VKH} 2$ & -010 & SIN & II & vo-198pa & & \\
\hline$\triangle 2 H 1 L$ & PNO & SIN & $i$ & & & \\
\hline$\triangle Z H I P$ & PND & SIN & II & & & \\
\hline $\begin{array}{l}42 \mathrm{HS} \\
42 \mathrm{HSS}\end{array}$ & $\begin{array}{l}\text {-TET } \\
\text { PND }\end{array}$ & $\begin{array}{l}\text { SIN } \\
\text { SIN }\end{array}$ & $\begin{array}{l}\text { II } \\
\text { II }\end{array}$ & $42 H 5 S O$ & & \\
\hline$D G-T S S$ & $R E C$ & & $\times 1$ & $\mathrm{D} 22 \mathrm{H} \oplus \mathrm{O}$ & & \\
\hline DK-Vs & DET & & $x \| 11$ & & & \\
\hline FEU $-R S$ & PHM & & XVI & & & \\
\hline$F S-K S$ & PMC & & $x v$ & & & \\
\hline$G-5$ & TRI & SIN & & $M 390$ & & \\
\hline$G-3 A$ & $T R I$ & $S$ IN & & GUSAD & & \\
\hline G-SRA & TRI & $\sin$ & & $6 U-58 a$ & & \\
\hline $6 I-58$ & $T R I$ & SIN & II I & & & \\
\hline GKSA & $\begin{array}{l}T R I \\
T E T\end{array}$ & SIN & IIt & & & \\
\hline $6 M 1-3$ & $T E T$ & SIN & 11 & & & \\
\hline $65-58$ & $T R I$ & SIN & & 643340 & & \\
\hline GUSA & TRI & SIN & II! & & & \\
\hline GU5B & TRI & $\operatorname{SIN}$ & III & & & \\
\hline $\begin{array}{r}\text { GUD - } \\
\text { LP-5 }\end{array}$ & $\begin{array}{l}\text { TR I } \\
\text { COM }\end{array}$ & $\begin{array}{l}\text { SIN } \\
\text { ACS }\end{array}$ & vII & 61200 & & \\
\hline$M T I-S$ & $\bullet \mathrm{RI}$ & THY & & $T G I-1-325160$ & & \\
\hline$O G-5$ & DEC & & $x \times 111$ & & & \\
\hline PSA & & & $x$ & & & \\
\hline PSB & & & $x$ & $2 N 107 *$ & & \\
\hline PSO & & & $x$ & CK $727 *$ & & \\
\hline PSG & & & $x$ & 2 N65* & & \\
\hline PSV & & & $x$ & & & \\
\hline PSYE & & & & & & \\
\hline $\begin{array}{r}R-5 \\
R 3-5\end{array}$ & & & $\begin{array}{l}x \times 11 \\
x \times 11\end{array}$ & & & \\
\hline$R B-5 A$ & & & $x \times 11$ & & & \\
\hline 5658 & 010 & SIN & v & & & \\
\hline $5 \operatorname{sos} 8-v$ & REG & & $v_{y=}$ & & & \\
\hline 5T5-5 & COU & & $\begin{array}{l}x \times ! \\
v i !\end{array}$ & & & \\
\hline TKH-SA & TRI & THY & vit & & & \\
\hline TV -5 & THM & & xvII! & & & \\
\hline$T V 8-5$ & THM & & $x \vee I I I$ & & & \\
\hline
\end{tabular}

\begin{tabular}{|c|c|c|c|c|c|}
\hline \multicolumn{6}{|c|}{ GROUP I. NUMERICAL } \\
\hline TYPE NUMBER & almo & IYPE & $\begin{array}{l}\text { IABLE } \\
\text { no. }\end{array}$ & SIKILAR TYPES & COST SPEC. \\
\hline $\begin{array}{l}U V-5 \\
V G-5\end{array}$ & $\begin{array}{l}\text { TWT } \\
\text { POW }\end{array}$ & & $\begin{array}{l}1 \times \\
x+1\end{array}$ & & \\
\hline $\begin{array}{l}\text { SLO } 18 \\
5 L 0381\end{array}$ & $\begin{array}{l}\text { DS } \\
\text { OS }\end{array}$ & & vitt & 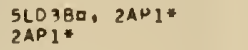 & \\
\hline SSRI & OS & & & SCPIA* & \\
\hline $5 S R 7$ & 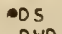 & & & SCP7A* & \\
\hline 5 TS3S & OWD & SIN & It & $5 U 46 *$ & $8360-57$ \\
\hline STS4 & $\begin{array}{l}010 \\
010\end{array}$ & $\begin{array}{l}\text { OUD } \\
\text { OUD }\end{array}$ & 11 & $3 T 5450,5246$. & \\
\hline $5 T 54.5$ & 010 & DUD & it & $324 *$ & $8079-56$ \\
\hline $\begin{array}{l}\text { STSBS } \\
\text { STS9SE }\end{array}$ & $\begin{array}{l}\text { DWD } \\
\text { DWD }\end{array}$ & SIN & It & & $8361-57$ \\
\hline \multirow{2}{*}{$\begin{array}{l}\text { STS95 } \\
\text { STS12P }\end{array}$} & DWD & SIN & it & 15020 & $8362-57$ \\
\hline & 010 & SIN & It & (20) & \\
\hline SVKMI & -OWD & SIN & & $3246 *$ & \\
\hline \multirow{2}{*}{$\begin{array}{l}\text { SVKH2 } \\
5 \mathrm{VKH} 3\end{array}$} & OWD & $\operatorname{SIN}$ & It & $3046 *$ & \\
\hline & ONO & SIN & II & $5 Y 36 *$ & \\
\hline$D 6$ & $R E G$ & & $x ! 1$ & & \\
\hline$D G-T S 6$ & $R E C$ & & $x 1$ & $\mathrm{D}_{2} \mathrm{Ka}$ & \\
\hline DK-V6 & DET & & $x \| I$ & & \\
\hline$F S-A 6$ & PHC & & $x V$ & & \\
\hline$F S=06$ & PHC & & XV & & \\
\hline FS-KG & PHC & & $x v$ & & \\
\hline $6 I-6 B$ & TRI & SIN & III & & \\
\hline GK6A & TRI & SIN & II I & & \\
\hline GMI-6 & BEA & TWN & I II & & \\
\hline $\begin{array}{r}G 56 \\
65-6\end{array}$ & $\begin{array}{l}\text { TRI } \\
\text { CDU }\end{array}$ & $\operatorname{SIN}$ & $\underset{x \times 1}{1}$ & & \\
\hline $\begin{array}{l}L D=6 \\
L I-6\end{array}$ & $\begin{array}{l}\text { TRI } \\
\text { IC }\end{array}$ & SIN & VIII & $6 I-68 d$ & \\
\hline MMT-6 & TMS & & $x 1 x$ & & \\
\hline $\begin{array}{l}\text { MS-6 } \\
\text { P6A }\end{array}$ & $\mathrm{COU}$ & & $\begin{array}{l}x \times 1 \\
x\end{array}$ & & \\
\hline P68 & & & $\hat{x}$ & & \\
\hline P60 & & & $x$ & & \\
\hline P6G & & & $x$ & & \\
\hline P6V & & & $x$ & & \\
\hline R6 & & & $x \times I I$ & & \\
\hline $5 T 5-6$ & CDU & & $x \times 1$ & & \\
\hline STSV-6 F-4 & PHD & & $x v$ & & \\
\hline$T S V-6 \quad F-3$ & PHD & & $x v$ & & \\
\hline TV8-6 & THM & & xvilt & & \\
\hline UV-6 & TWT & & $1 x$ & & \\
\hline Vs-6 & COU & & $x \times 1$ & & \\
\hline 6A2P & PTG & SIN & II & $68 E 6 *$, tK90* & $8354 b 1$ \\
\hline $\begin{array}{l}\text { 6A3P } \\
6 A 5 B \\
6 A 6 A\end{array}$ & $\begin{array}{l}\text { GTB } \\
\text { PTG } \\
\text { OIO }\end{array}$ & $\begin{array}{l}\operatorname{SIN} \\
\operatorname{SIN}\end{array}$ & 11 & $\begin{array}{l}\text { 6BN6* } \\
667 *\end{array}$ & \\
\hline 6A7 & PTG & SIN & II & 6.5A 7* & $8086-56$ \\
\hline $6 A B$ & PTG & SIN & II & $6 A 8 B 0,6 A B *$ & $8367-57$ \\
\hline 6ABB & PTG & SIN & & 6AB* & \\
\hline 6ABY & APTG & $\sin$ & & 6ABS०D & \\
\hline GAIOS & PTG & SIN & 11 & 65A7* & $8087-56$ \\
\hline $6 A 158$ & PPTG & $\operatorname{SIN}$ & & $6547 *$ & \\
\hline $6 A G 7$ & ๑BEA & SIN & & $6 P 90,6 A G 7 *$ & \\
\hline $\begin{array}{l}\text { GAZHS } \\
\text { SBIP }\end{array}$ & $\begin{array}{l}\text { PPNO } \\
\text { PND }\end{array}$ & $\begin{array}{l}\text { S1N } \\
010\end{array}$ & 11 & $6 A G 5 \%, E F 96 \%$ & \\
\hline $682 P$ & PNO & DIO & 11 & 110000 & $\therefore$ \\
\hline 684 & -TRI & SIN & & $6 A 3 *$ & ? \\
\hline 688 & PND & OWD & & $688 *, 6886,688 M$ & \\
\hline $688 \%$ & $\triangle P N D$ & OWD & & 6B85口, 688G* & \\
\hline 6885 & PNO & DWD & II & $6 B 8 G, 688 M e$ & $8369-57$ \\
\hline $\begin{array}{l}\text { 68KH1 } \\
\text { 601A }\end{array}$ & $\begin{array}{l}-010 \\
010\end{array}$ & $\begin{array}{l}\text { DUD } \\
\text { SIN }\end{array}$ & & $\begin{array}{l}\text { 6KH5SD } \\
\text { 6D6A\&, } \quad 3704 \%\end{array}$ & \\
\hline $6012 \mathrm{H}$ & $\triangle 010$ & $\operatorname{SIN}$ & & $6042 \mathrm{HA}, 9004 *$ & \\
\hline 6030 & 010 & $\operatorname{SIN}$ & I 1 & $559 *$ & \\
\hline $6042 \mathrm{H}$ & 010 & $\operatorname{Sin}$ & 11 & 9004 & \\
\hline $606 \mathrm{~A}$ & 010 & SIN & II & $3704 \%, 0601140$ & \\
\hline 6080 & 010 & SIN & 11 & & \\
\hline 60100 & 010 & & II & & \\
\hline $\begin{array}{l}\text { 6016P } \\
\text { 6E5P }\end{array}$ & $\begin{array}{l}\text { DIO } \\
\text { TET }\end{array}$ & $\operatorname{SIN}$ & i1 & & \\
\hline 6E5P & TET & SIN & II & & \\
\hline
\end{tabular}




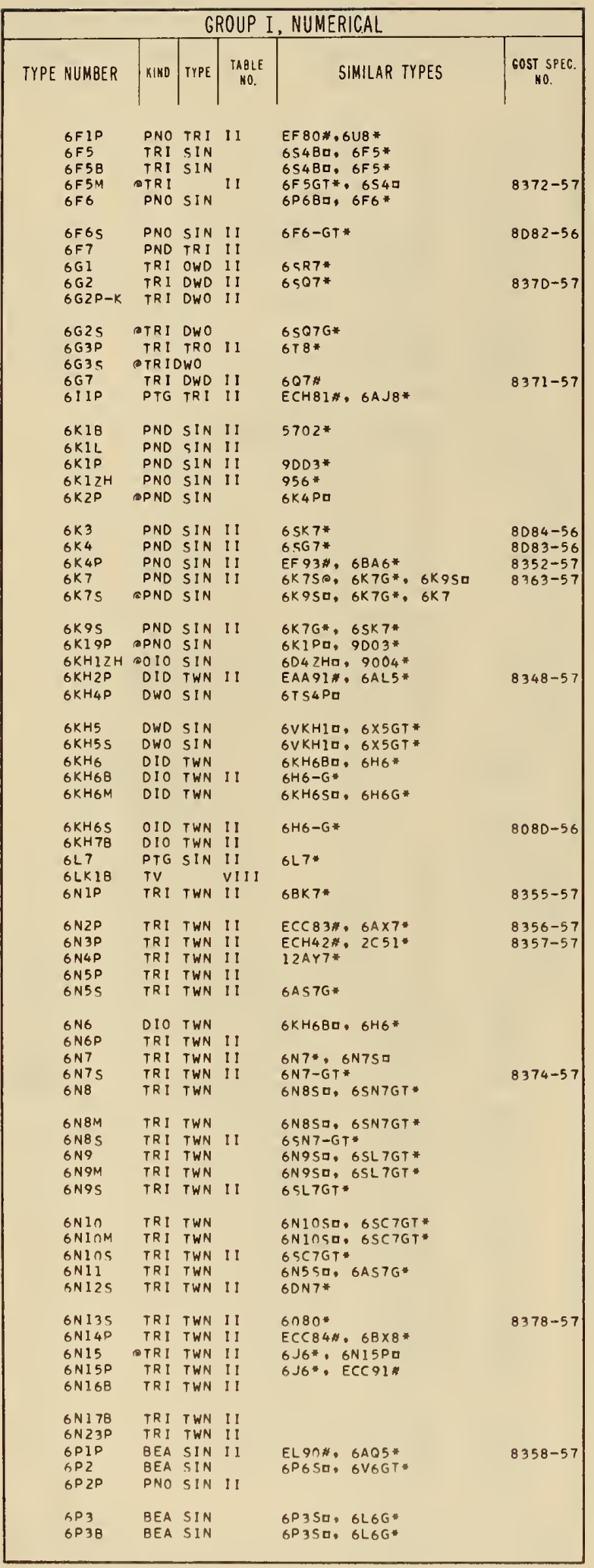

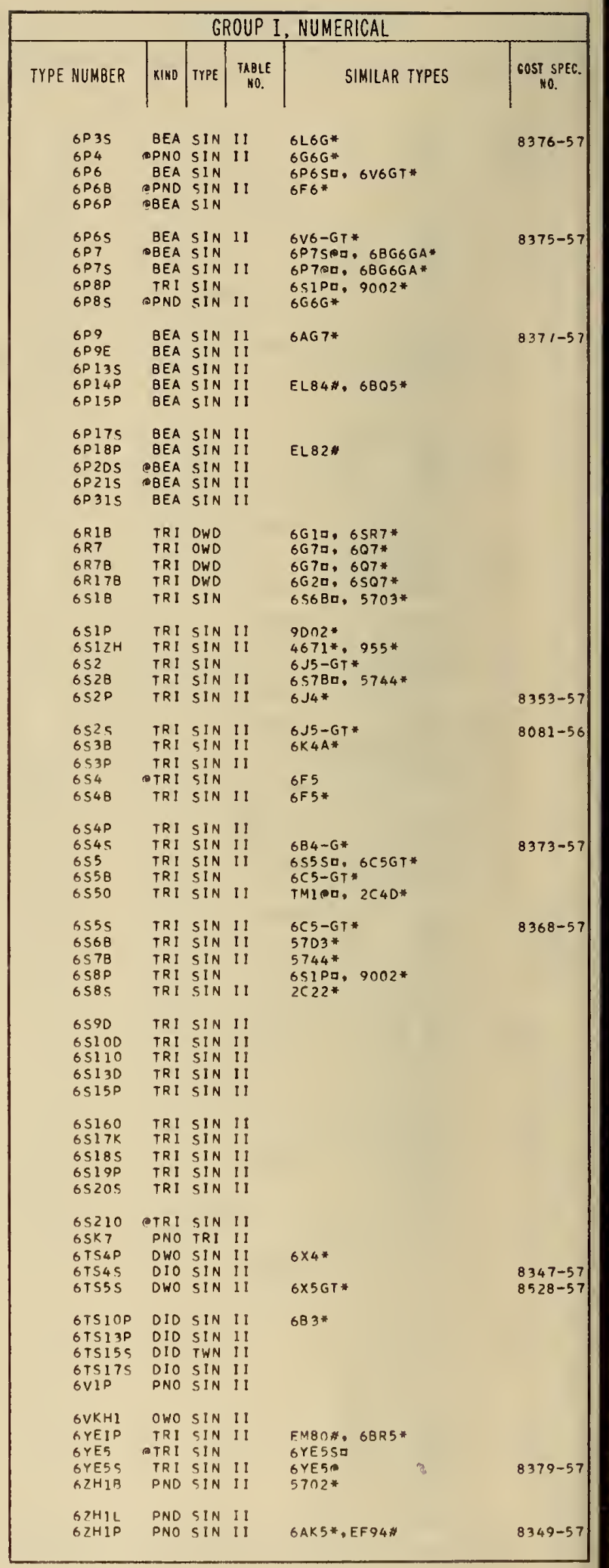




\begin{tabular}{|c|c|c|c|c|c|}
\hline \multicolumn{6}{|c|}{ GROUP I. NUMERICAL } \\
\hline TYPE NLYAER & $11 \times 0$ & IYPE & $\begin{array}{c}\text { IASLE } \\
\text { No. }\end{array}$ & SIMILAR TYPES & COSI SPEC \\
\hline $62 \mathrm{H}: 2 \mathrm{H}$ & PND & $\operatorname{SIN}$ & I' & 954 & \\
\hline $52 \mathrm{H} 2 \mathrm{~B}$ & DNO & SIN & II & $5784.5630 *$ & \\
\hline $62 \mathrm{H}_{24}$ & PNO & $\operatorname{SIN}$ & II & $1851 *$ & \\
\hline $62 \mathrm{H} 2{ }^{\circ}$ & DNO & $\sin$ & 11 & 6456 & \\
\hline $82 \mathrm{H}$ & PND & SIN & II & 6.5H7. & $8085-58$ \\
\hline $82 \mathrm{H} 36$ & - OND & $\sin$ & [I & $6 A B>/ 1853$ & \\
\hline $62 \mathrm{H} 3 \mathrm{P}$ & PND & SIN & II & 64G5: EF96" & $8350-57$ \\
\hline $62 \mathrm{H}_{4}$ & ONO & SIN & II & $6 A C 7$ & $8364-57$ \\
\hline$B Z \mathrm{H} \triangle \mathrm{B}$ & -PND & $\operatorname{SIN}$ & & SAG7: & \\
\hline $62 \mathrm{H} \triangle \mathrm{E}$ & OND & SIN & II & & \\
\hline $62 \mathrm{H} \rightarrow \mathrm{P}$ & PNO & SIN & II & SAU6*, EF94* & \\
\hline $62^{\mathrm{HS}}$ & -TRI & SIN & & 635 & \\
\hline G2H5A & APNO & SIN & I I & & \\
\hline $62 \mathrm{H} 5 \mathrm{~B}$ & PND & $\sin$ & II & & \\
\hline $62 H 50$ & BEA & SIN & I I & GAH6* & $8351-57$ \\
\hline $82 \mathrm{HSO}$ & -PNO & SIN & & $6 J 7 *$ & \\
\hline $62 \mathrm{H} 6 \mathrm{~S}$ & PNO & $\operatorname{Sin}$ & I! & $262 \%$ & \\
\hline $62 \mathrm{H7}$ & PNO & $\operatorname{SIN}$ & II & 637 & $8365-57$ \\
\hline $62 \mathrm{H} 78$ & - DNO & SIN & & $6 W 7 G$ & \\
\hline $62 \times 8$ & OND & SIN & 【I & $65 \mathrm{J7}$. & $8366-57$ \\
\hline $6 Z^{4} 85$ & PNO & $\operatorname{SIN}$ & II & & \\
\hline $62 \mathrm{H} 9 \mathrm{~B}$ & ONO & SIN & II & & \\
\hline 6ZHQP & PNO & $S I N$ & II & & \\
\hline $62 \mathrm{H} 10 \mathrm{~B}$ & PNO & $\operatorname{SIN}$ & I I & & \\
\hline $62 H 10 P$ & PNO & SIN & II & & \\
\hline $62 \mathrm{H} 118$ & ๑NO & $\operatorname{SIN}$ & & $6547=$ & \\
\hline $52 \mathrm{H} 11 \mathrm{P}$ & PNO & SIN & II & 6805 & \\
\hline $62 \mathrm{H} 128$ & -PND & $S I N$ & & $6 \leq G 7 \%$ & \\
\hline $62 \mathrm{H}_{13}$ & PNO & SIN & & $62 \mathrm{H} 13 \mathrm{LO}$ & \\
\hline $62 \mathrm{H} 13 \mathrm{~L}$ & PNO & SIN & II & 62413 & \\
\hline $6 Z \mathrm{H} 2 \mathrm{OP}$ & BEA & 010 & II & & \\
\hline $62 \mathrm{H} 210$ & $B E A$ & 010 & It & & \\
\hline $62 \mathrm{H} 22 \mathrm{P}$ & 010 & BEA & II & & \\
\hline $62 \mathrm{H} 23{ }^{\circ}$ & PNO & OBA & II & & \\
\hline $62 \mathrm{H} 32 \mathrm{P}$ & PNO & SIN & II & & \\
\hline 07 & REG & & $x I I$ & & \\
\hline 074 & $R \subseteq C$ & & XI & $D G=T S 2100$ & \\
\hline 078 & REC & & $x I$ & $D G-T S 2290$ & \\
\hline 070 & REC & & $\times !$ & $O G-T 525 \circ \square$ & \\
\hline 076 & REC & & $x I$ & $O G=T S 240 \mathrm{C}$ & \\
\hline $07 \mathrm{~V}$ & REC & & $\begin{array}{l}x ! \\
x !\end{array}$ & $O G=T 523 \bullet \square$ & \\
\hline $\begin{array}{l}\text { DTYE } \\
\text { OTZH }\end{array}$ & REC & & $\begin{array}{l}x I \\
X I\end{array}$ & $O G-T S 27 e \square$ & \\
\hline $\begin{array}{l}072 \mathrm{H} \\
0 \mathrm{G}-T 57\end{array}$ & $\begin{array}{l}\text { REC } \\
\text { REC }\end{array}$ & & $\begin{array}{l}x I \\
x I\end{array}$ & $\begin{array}{ll}0,40 \\
\end{array}$ & \\
\hline $\begin{array}{r}D G-T S 7 \\
D K-57\end{array}$ & $\begin{array}{l}\text { REC } \\
\text { MIX }\end{array}$ & & $x I I I$ & & \\
\hline$D K-V 7$ & OET & & $x \in I$ & & \\
\hline$F S=K 7$ & PHC & & $x V$ & & \\
\hline $\begin{aligned} G I & =78 \\
G M I & =7\end{aligned}$ & TRI & $\sin$ & II & & \\
\hline $\begin{array}{r}G M I-7 \\
\text { GS }-7\end{array}$ & $\begin{array}{l}\text { TET } \\
\text { COU }\end{array}$ & $\operatorname{SIN}$ & $\begin{array}{l}\text { III } \\
x \times 1\end{array}$ & & \\
\hline $\begin{array}{l}G S-7 \\
G S-7 A\end{array}$ & $\begin{array}{l}\text { TRI } \\
\text { TRI }\end{array}$ & $\begin{array}{l}\sin \\
\sin \end{array}$ & Itt & $G K-3 \cap 000$ & \\
\hline $6 s-78$ & TRI & $\begin{array}{l}\operatorname{SIN} \\
\operatorname{SIN}\end{array}$ & itt & & \\
\hline$<5-7$ & TR I & $\operatorname{SIN}$ & & $G-8110,811-A *$ & \\
\hline$L O-7$ & TR I & SIN & & $6 I-78 \square$ & \\
\hline LI I-7 & IC & & VIII & & \\
\hline $4 S-7$ & $\mathrm{COU}$ & & $x \times 1$ & & \\
\hline P7 & & & $x$ & & \\
\hline$R=7$ & & & $x \times I I$ & & \\
\hline $5 G 7 S$ & DIO & $\operatorname{SIN}$ & v & & \\
\hline$T \vee B-7$ & THM & & XVIII & & \\
\hline$U V-7$ & $T W T$ & & $I x$ & & \\
\hline 7L01M & os & & VI]I & & \\
\hline $7 L 0551$ & os & & VIII & $34 P_{1}$ * & \\
\hline $7 P 125$ & PND & $\sin$ & II & & \\
\hline $72 \mathrm{H} 12 \mathrm{~S}$ & DND & $\sin$ & II & $328 \mathrm{~A}$ & \\
\hline 08 & REG & & $x ! 1$ & & \\
\hline$D G-T \$ 8$ & REC & & $\begin{array}{l}x I \\
x y\end{array}$ & $02 V a$ & \\
\hline$F S-K 8$ & PHC & & $\begin{array}{l}x V \\
I 11\end{array}$ & PI-30กO & \\
\hline$G I-8$ & PNO & SIN & 111 & & \\
\hline GS-8 & $\mathrm{COU}$ & & $x \times I$ & & \\
\hline Gu8 & TRI & $\operatorname{SIN}$ & III & & $7711-55$ \\
\hline
\end{tabular}

\begin{tabular}{|c|c|c|c|c|c|}
\hline \multicolumn{6}{|c|}{ GROUP I. NUMERICAL } \\
\hline TYPE NUMBER & NINO & IYPE & $\begin{array}{l}\text { TABLE } \\
\text { NO. }\end{array}$ & SIMILAR TYPES & $\cos :$ or: \\
\hline KMT -8 & TMS & & $x I x$ & & \\
\hline MMT -8 & TMS & & $x I x$ & & \\
\hline$M S-8$ & cou & & $x \times I$ & & \\
\hline P8 & & & $x$ & & \\
\hline P8A & & & $x$ & & \\
\hline$R-8$ & & & $x \times 11$ & & \\
\hline $568 S$ & 010 & $\sin$ & v & & \\
\hline STS -8 & COU & & $x \times 1$ & & \\
\hline$T 80$ & TMS & & $x I x$ & & \\
\hline T8E & TMS & & $\hat{x} I \hat{x}$ & & \\
\hline$T 8 M$ & TMS & & $x I x$ & & \\
\hline$T 8 R$ & TMS & & $\hat{x} \mathbb{x}$ & & \\
\hline T8S1 & TMS & & $x I x$ & & \\
\hline T8SIM & TMS & & $x+\hat{x}$ & & \\
\hline$T 8 \mathrm{S2}$ & TMS & & $\hat{x} I \hat{x}$ & & \\
\hline T852M & TMS & & $x I x$ & & \\
\hline$T 853$ & TMS & & $\hat{x} I \hat{x}$ & & \\
\hline T8S3M & TMS & & $x I x$ & & \\
\hline$r G 8 / 3$ & TR I & THY & VII & $T G 1-2.5 / 4 \square$ & \\
\hline$T \vee B-8$ & THM & & XVIII & & \\
\hline vs -8 & $\mathrm{COU}$ & & $x \times I$ & & \\
\hline 8LM3V & OS & & VIII & & \\
\hline $8 L 028$ & OS & & & 8LO29ם, 3BP1A* & \\
\hline $8 L 0291$ & os & & VIII & $38 P_{1 *} *$ & \\
\hline $8 L 029 M$ & OS & & VIII & & \\
\hline $8 \mathrm{LO} 30 \mathrm{I}$ & 0.5 & & VIII & $30 P_{1} *$ & \\
\hline $8 \mathrm{LO} 30 \mathrm{M}$ & OS & & VIII & & \\
\hline $8 L 039 \mathrm{~V}$ & os & & VIII & 3 JP7 * & \\
\hline$O Q A$ & REC & & $x I$ & & \\
\hline 098 & REC & & $x i$ & & \\
\hline 090 & REC & & $x I$ & & \\
\hline DQG & REC & & $x !$ & & \\
\hline 091 & REC & & $x I$ & & \\
\hline $\begin{array}{l}\text { OQKK } \\
\text { OQLL }\end{array}$ & $\begin{array}{l}\text { REC } \\
\text { REC }\end{array}$ & & $\begin{array}{l}x I \\
\times 1\end{array}$ & & \\
\hline OQV & REC & & $x !$ & & \\
\hline OQYE & REC & & $\hat{x I}$ & & \\
\hline OQZH & REC & & $x I$ & & \\
\hline DG-TSQ & REC & & $\mathrm{xI}$ & D2A口 & \\
\hline$G-9$ & $T R I$ & SIN & & GIJ650 & \\
\hline $\mathrm{GS}=9$ & $\mathrm{cou}$ & & $x \times I$ & & \\
\hline$G S 9 B$ & TRI & SI v & II I & & \\
\hline LD-9 & TRI & SIN & & $G S-9 B d$ & \\
\hline MMT $=9$ & TMS & & $x I x$ & & \\
\hline$M S-9$ & cou & & $x \times 1$ & & \\
\hline PQ & & & $x$ & 2 N $35 *$ & \\
\hline PQA & & & $\hat{x}$ & & \\
\hline$R=9$ & & & $\hat{x} \times 11$ & & \\
\hline SG9S & 010 & $\operatorname{SIN}$ & v & & \\
\hline STSV-9 $\quad F-1$ & PHO & & $x V$ & & \\
\hline T9 & TMS & & $x \mid x$ & & \\
\hline TV $B-9$ & THM & & XVIII & & \\
\hline vs-9 & cou & & $x \times 1$ & & \\
\hline $\begin{array}{l}\text { OIn } \\
\text { DIDA }\end{array}$ & $\begin{array}{l}\text { REC } \\
\text { REC }\end{array}$ & & $\begin{array}{l}x I \\
\times I\end{array}$ & & \\
\hline DIDB & REC & & $x I$ & & \\
\hline DGTSIO & REC & & $x !$ & $028 a$ & \\
\hline Gio & TRI & $\operatorname{SIN}$ & III & & \\
\hline$G-10 \mathrm{~A}$ & TRI & $\sin$ & & $G U-\ln A \square$ & \\
\hline G-IORA & TRI & SIN & & Gu-1080 & \\
\hline $\begin{array}{r}G K O-10 \\
G S-10\end{array}$ & $\begin{array}{l}\text { TRI } \\
\text { COU }\end{array}$ & $\sin$ & $x \times 1$ & $G K-2000 a$ & \\
\hline$G T-10$ & TRI & SIN & & G460 & \\
\hline GUIกA & TRI & SIN & III & & \\
\hline GUIDB & $T R I$ & SIN & III & & \\
\hline I SK 10 & & & $x x$ & & \\
\hline 15010 & & & $x x$ & & \\
\hline IST 10 & & & $x x$ & & \\
\hline KMT -10 & TMS & & $x I x$ & & \\
\hline$M O-10$ & $T R I$ & $\operatorname{SIN}$ & III & & \\
\hline $\begin{array}{r}D 10 \\
R=10\end{array}$ & & & $\begin{array}{l}x \\
x \times 11\end{array}$ & $2 N 35$ & \\
\hline
\end{tabular}




\begin{tabular}{|c|c|c|c|c|c|}
\hline \multicolumn{6}{|c|}{ GROUP I, NUMERICAL } \\
\hline TYPE NUMBER & KINO & TYPE & $\begin{array}{l}\text { TABLE } \\
\text { NO. }\end{array}$ & SIMILAR TYPES & $\begin{array}{c}\text { COST SPEC. } \\
\text { HO. }\end{array}$ \\
\hline $\begin{array}{l}\text { SG1OS } \\
\text { TO-10 } \\
V G-10 \\
\text { VG-1D-30 } \\
V G-10-45\end{array}$ & $\begin{array}{l}\text { REG } \\
\text { PNO } \\
\text { POW } \\
\text { POW } \\
\text { POW }\end{array}$ & SIN & $\begin{array}{l}v \\
x I 1 \\
x I 1 \\
x I 1\end{array}$ & $10 P 125$ & \\
\hline $\begin{array}{l}V G-1 D-55 \\
V G-10-80 \\
V G-10-110 \\
V G-1 D-15 D \\
V K=10\end{array}$ & $\begin{array}{l}\text { POW } \\
\text { POW } \\
\text { POW } \\
\text { POW } \\
\text { POW }\end{array}$ & & 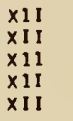 & & \\
\hline $\begin{array}{l}10 L K 2 B \\
10 L O 43 I \\
10 P 12 S \\
10 Z H 1 L \\
102 H 3 L\end{array}$ & $\begin{array}{l}\text { PR } \\
\text { OD } \\
\text { PNO } \\
\text { PND } \\
\text { PND }\end{array}$ & $\begin{array}{l}\text { SIN } \\
\text { SIN } \\
\text { SIN }\end{array}$ & $\begin{array}{l}\text { V111 } \\
\text { VIII } \\
\text { II } \\
\text { II } \\
\text { II }\end{array}$ & $\begin{array}{l}\text { 10ZH3L } \\
10 Z H 1 L \square\end{array}$ & \\
\hline $\begin{array}{c}10 Z H 12 S \\
011 \\
F E U=11 \\
G I-118 \\
G S-11\end{array}$ & $\begin{array}{l}\text { PND } \\
\text { REC } \\
\text { PHM } \\
\text { TRI } \\
\text { COU }\end{array}$ & $\operatorname{SIN}$ & $\begin{array}{l}I 1 \\
X I \\
X V I \\
I 1 I \\
X X 1\end{array}$ & $310 A *$ & \\
\hline $\begin{array}{l}\text { GU11A } \\
\text { GU118 } \\
\text { KMT-11 } \\
\text { LO11 } \\
\text { MS-11 }\end{array}$ & $\begin{array}{l}\text { TRI } \\
\text { TRI } \\
\text { TMS } \\
\text { TRI } \\
\text { COU }\end{array}$ & $\begin{array}{l}\operatorname{SIN} \\
\operatorname{SIN} \\
\operatorname{SIN}\end{array}$ & $\begin{array}{l}111 \\
\text { III } \\
x I X \\
x x I\end{array}$ & $G I-118 \square$ & \\
\hline $\begin{array}{r}P 11 \\
R-11 \\
V S-11 \\
012 \\
012 A\end{array}$ & $\begin{array}{l}\text { COU } \\
\text { REC } \\
\text { REC }\end{array}$ & & $\begin{array}{l}x \\
x \times 11 \\
x \times I \\
x I \\
x 1\end{array}$ & & \\
\hline $\begin{array}{l}\text { OGTS12 } \\
\text { FEU-12 } \\
\text { GI-12B } \\
\text { GS-12 } \\
\text { GU12A }\end{array}$ & $\begin{array}{l}\text { REC } \\
\text { PHM } \\
\text { TRI } \\
\text { COU } \\
\text { TRI }\end{array}$ & $\operatorname{SIN}$ & $\begin{array}{l}x I \\
X V I \\
1 I I \\
X X I \\
I I I\end{array}$ & $880 *$ & \\
\hline $\begin{array}{l}\text { KMT-12 } \\
\text { LO12 } \\
M M T-12 \\
M S-12 \\
0 \$ 12 / 500\end{array}$ & $\begin{array}{l}\text { TMS } \\
\text { TRI } \\
\text { TMS } \\
\text { COU } \\
\text { PPND }\end{array}$ & $\operatorname{SIN}$ & $\begin{array}{l}x I x \\
x I X \\
x \times I\end{array}$ & $\begin{array}{l}G !-128 \square \\
G B 37 *\end{array}$ & \\
\hline $\begin{array}{l}P 12 \\
R-12 \\
1281 M \\
1282 M \\
12 G 1\end{array}$ & $\begin{array}{l}\text { PND } \\
\text { PND } \\
\text { TRI }\end{array}$ & $\begin{array}{l}\text { OWD } \\
\text { OWD } \\
\text { OWD }\end{array}$ & $\begin{array}{l}x \\
x x I 1 \\
\text { II } \\
\text { II } \\
\text { II }\end{array}$ & $12 S R 7 *$ & \\
\hline $\begin{array}{l}1262 \\
12 K 1 M \\
12 K 3 \\
12 K 4 \\
12 K 128\end{array}$ & $\begin{array}{l}\text { TRI } \\
\text { PNO } \\
\text { PNO } \\
\text { PNO } \\
\text { PNO }\end{array}$ & $\begin{array}{l}\text { OWO } \\
\text { SIN } \\
\text { SIN } \\
\text { SIN } \\
\text { SIN }\end{array}$ & $\begin{array}{l}\text { II } \\
\text { II } \\
\text { II } \\
\text { II }\end{array}$ & $\begin{array}{l}12507 * \\
125 K 7 * \\
12567 * \\
12 K 4 \square, 12567 *\end{array}$ & \\
\hline $\begin{array}{l}12 K 17 \mathrm{~B} \\
12 \mathrm{KH} 35 \\
12 \mathrm{MIM} \\
12 \mathrm{~N} 1 \\
12 \mathrm{~N} 4 \mathrm{P}\end{array}$ & $\begin{array}{l}\text { PND } \\
\text { OWO } \\
\text { PND } \\
\text { TRI } \\
\text { TRI }\end{array}$ & $\begin{array}{l}\text { SIN } \\
\text { SIN } \\
\text { TRI } \\
\text { TWN } \\
\text { TWN }\end{array}$ & $\begin{array}{l}\text { II } \\
\text { II } \\
\text { II }\end{array}$ & $\begin{array}{l}12 K 3 \mathrm{~B}, 12 S K 7^{*} \\
\text { LG1 } \\
12 \mathrm{~N} 11 \mathrm{SB}, 12 \mathrm{AH} 7 \mathrm{~T} \text { * } \\
12 \mathrm{AY} 7 *\end{array}$ & \\
\hline $\begin{array}{l}12 N 10 \\
12 N 10 M \\
12 N 105 \\
12 N 115 \\
12 P 45\end{array}$ & $\begin{array}{l}\text { TRI } \\
\text { TRI } \\
\text { TRI } \\
\text { TRI } \\
\text { PND }\end{array}$ & $\begin{array}{l}\text { TWN } \\
\text { TWN } \\
\text { DUO } \\
\text { TWN } \\
\text { S IN }\end{array}$ & $\begin{array}{l}11 \\
\text { II } \\
\text { II }\end{array}$ & $\begin{array}{l}12 N 10 S \square, \quad 12 S C 7 G T * \\
12 N 10 S \square, 12 S C 7 G T * \\
12 S C 7 * \\
12 A H 7 G T *\end{array}$ & \\
\hline $\begin{array}{l}12 P 145 \\
12 P 17 L \\
12 R 18 \\
12 R 178 \\
1252\end{array}$ & $\begin{array}{l}\text { BEA } \\
\text { PND } \\
\text { TRI } \\
\text { TRI } \\
\text { PTRI }\end{array}$ & $\begin{array}{l}\text { SIN } \\
\text { SIN } \\
\text { OWO } \\
\text { DWO } \\
\text { SIN }\end{array}$ & $\begin{array}{l}\text { II } \\
\text { II } \\
\text { II }\end{array}$ & $\begin{array}{l}12610,125 R 7 * \\
1262 \square, 12507 *\end{array}$ & \\
\hline $\begin{array}{l}1253 \mathrm{~S} \\
12 \mathrm{HL} \\
12 \mathrm{H} 1 \mathrm{~L} \\
12 \mathrm{ZHIM} \\
122 \mathrm{H} 3 \mathrm{~L}\end{array}$ & $\begin{array}{l}-T R I \\
\text { PPND } \\
\text { PND } \\
\text { PND } \\
\text { PNO }\end{array}$ & $\begin{array}{l}\operatorname{SIN} \\
\operatorname{SIN} \\
\operatorname{SIN} \\
\operatorname{SIN} \\
\operatorname{Sin}\end{array}$ & $\begin{array}{l}\text { II } \\
\text { I I } \\
\text { II } \\
\text { II }\end{array}$ & $\begin{array}{l}\text { LO1व } \\
12 Z H 1 L \square \\
12 Z H 1\end{array}$ & \\
\hline $\begin{array}{l}122 \mathrm{ZH} \\
12 \mathrm{ZH} 178\end{array}$ & $\begin{array}{l}\text { PNO } \\
\text { PNO }\end{array}$ & $\begin{array}{l}\operatorname{SIN} \\
\operatorname{SIN}\end{array}$ & II & $\begin{array}{l}125 J 7 * \\
12 Z H 8 \square, 12 S J 7 *\end{array}$ & \\
\hline
\end{tabular}

\begin{tabular}{|c|c|c|c|c|c|}
\hline \multicolumn{6}{|c|}{ GROUP I, NUMERICAL } \\
\hline TYPE NUMBER & KINO & TYPE & $\begin{array}{c}\text { TABLE } \\
\text { NO. }\end{array}$ & SIMILAR TYPES & $\begin{array}{c}\text { Cost SPEC. } \\
\text { NO. }\end{array}$ \\
\hline $\begin{array}{r}013 \\
\text { OGTS13 } \\
F E U-13 \\
G-13 \\
G I-13\end{array}$ & $\begin{array}{l}\text { REC } \\
\text { REC } \\
\text { PHM } \\
\text { TRI } \\
\text { TRI }\end{array}$ & $\begin{array}{l}\operatorname{SIN} \\
\operatorname{SIN}\end{array}$ & $\begin{array}{l}X I \\
X I \\
X V I \\
I I I \\
I I I\end{array}$ & & \\
\hline $\begin{array}{l}G 1-13 B \\
G M 13 \\
G U 13 \\
L I-13 \\
M S-13\end{array}$ & $\begin{array}{l}\text { TRI } \\
\text { TET } \\
B E A \\
\text { IM } \\
\text { COU }\end{array}$ & $\begin{array}{l}\operatorname{SIN} \\
\operatorname{SIN} \\
\operatorname{SIN}\end{array}$ & $\begin{array}{l}\text { III } \\
\text { III } \\
\text { III } \\
\text { VIII } \\
x \times I\end{array}$ & 813* & \\
\hline $\begin{array}{l}\text { P13 } \\
\text { P13A } \\
\text { P13B }\end{array}$ & & & $\begin{array}{l}x \\
x \\
x\end{array}$ & $\begin{array}{l}2 \mathrm{~N} 43 * \\
2 \mathrm{~N} 34 *\end{array}$ & \\
\hline $\begin{array}{l}S G 13 P \\
V 13 / 30\end{array}$ & $\begin{array}{r}010 \\
010 \\
010\end{array}$ & $\begin{array}{l}\operatorname{SIN} \\
\operatorname{SIN}\end{array}$ & $\begin{array}{l}\hat{v} \\
\text { IV }\end{array}$ & $v_{1}-00_{3} / 13 \square$ & \\
\hline $\begin{array}{c}\text { VS-13 } \\
13 L K 18 \\
13 L K 2 B \\
13 L M 4 V \\
13 L M 31 M\end{array}$ & $\begin{array}{l}\text { cov } \\
\text { TV } \\
\text { TV } \\
\text { OS } \\
\text { os }\end{array}$ & & $\begin{array}{l}x x I \\
\text { VIII } \\
\text { VIII } \\
\text { VIII } \\
\text { VIIII }\end{array}$ & $\begin{array}{l}\text { 5FP4* } \\
\text { 5FP7* }\end{array}$ & \\
\hline $\begin{array}{l}13 \text { LM31V } \\
13 \text { LMS6I } \\
13 \text { LMS } \\
13 \text { LM570 } \\
13 \text { LKS } 58 K\end{array}$ & $\begin{array}{l}\text { os } \\
\text { os } \\
\text { os } \\
\text { os } \\
\text { os }\end{array}$ & & $\begin{array}{l}\text { VIII } \\
\text { VIII } \\
\text { VIII } \\
\text { VIII } \\
\text { VIII }\end{array}$ & $\begin{array}{l}\text { 5FP I* } \\
5 F P 7 *\end{array}$ & \\
\hline $\begin{array}{l}13 L 018 \\
13 L 028 \\
13 L 031 \\
13 L 041 \\
13 L 05 \mathrm{P}\end{array}$ & $\begin{array}{l}\text { os } \\
\text { os }\end{array}$ & . & $\begin{array}{l}\text { VIII } \\
\text { VIII } \\
\text { VIII } \\
\text { VIII } \\
\text { VIII }\end{array}$ & $\begin{array}{l}5 C P 1-A * \\
5 C P 7-A *\end{array}$ & \\
\hline $\begin{array}{l}13 L 06 P \\
13 L 036 \\
13 L 036 V \\
13 L 037 A \\
13 L 037 I\end{array}$ & $\begin{array}{l}\text { os } \\
\text { os } \\
\text { os } \\
\text { os }\end{array}$ & 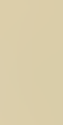 & $\begin{array}{l}\text { VIII } \\
\text { VIII } \\
\text { VIII } \\
\text { VIII } \\
\text { VIII }\end{array}$ & $\begin{array}{l}5 F P 7-A * \\
5 F \cdot P 7 * \\
5 C P_{1 *}\end{array}$ & \\
\hline $\begin{array}{l}13 L 037 M \\
13 L 048 A \\
13 L 048 I \\
13 L 048 M \\
13 L 054 A\end{array}$ & $\begin{array}{l}\text { OS } \\
00 \\
00 \\
00 \\
0 S\end{array}$ & - & $\begin{array}{l}\text { VIII } \\
\text { VIIII } \\
\text { VIIII } \\
\text { VIII } \\
\text { VIII }\end{array}$ & $5 S P_{1} *$ & \\
\hline $\begin{array}{l}13 L 054 M \\
13 L 054 \mathrm{~V} \\
13 \mathrm{LO} 101 \mathrm{M} \\
13 \mathrm{LO} 102 \mathrm{M} \\
13 \mathrm{LO} 104 \mathrm{~A}\end{array}$ & $\begin{array}{l}\text { OS } \\
\text { os } \\
\text { M TV }\end{array}$ & 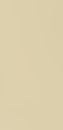 & $\begin{array}{l}\text { VIII } \\
\text { VIII } \\
\text { VIII } \\
\text { VIII } \\
\text { VIII }\end{array}$ & & \\
\hline $\begin{array}{l}13 P 1 \\
13 P 1 M \\
13 P 15 \\
014 \\
014 A\end{array}$ & $\begin{array}{l}\text { PBEA } \\
\text { BEA } \\
\text { BEA } \\
\text { REC } \\
\text { REC }\end{array}$ & $\begin{array}{l}\operatorname{SIN} \\
\operatorname{SIN} \\
\operatorname{SIN}\end{array}$ & $\begin{array}{r} \\
11 \\
\times 1 \\
X 1\end{array}$ & 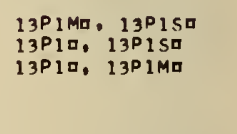 & \\
\hline $\begin{array}{l}\text { OGTS14 } \\
\text { FEU-14 } \\
\text { GI-14B } \\
\text { LI-14 } \\
M S-14\end{array}$ & $\begin{array}{l}\text { REC } \\
\text { PHM } \\
\text { TRI } \\
\text { IM } \\
\text { COU }\end{array}$ & SIN & $\begin{array}{l}x I \\
X V I \\
I 1 I \\
V I I I \\
x X I\end{array}$ & & \\
\hline $\begin{array}{r}P 14 \\
T V-14 \\
V S-14 \\
\text { OGTS15 } \\
\text { FEU }-15\end{array}$ & $\begin{array}{l}\text { THM } \\
\text { COU } \\
\text { REC } \\
\text { PHM }\end{array}$ & & $\begin{array}{l}x \\
X \vee I I I \\
X X I \\
X I \\
X \vee I\end{array}$ & $\begin{array}{l}\text { 2N65* } \\
\text { O2Na }\end{array}$ & \\
\hline $\begin{array}{l}\text { G-15A } \\
\text { G-15RA } \\
\text { GDO-15 } \\
\text { GU15 } \\
\text { IFK15-1 }\end{array}$ & $\begin{array}{l}\text { TRI } \\
\text { TRI } \\
\text { TRI } \\
\text { BEA }\end{array}$ & $\begin{array}{l}\operatorname{SIN} \\
\operatorname{SIN} \\
\operatorname{SIN} \\
\operatorname{SIN}\end{array}$ & $\begin{array}{l}111 \\
x x\end{array}$ & $\begin{array}{l}G U-11 A \square \\
G U-16 B \square \\
G-610\end{array}$ & \\
\hline $\begin{array}{c}\text { ISSH15 } \\
L I-15 \\
P 15 \\
\text { SG15P } \\
T G=15 / 3\end{array}$ & $\begin{array}{l}\text { IM } \\
\text { DIO } \\
\text { TRI }\end{array}$ & $\begin{array}{l}\text { SIN } \\
\text { THY }\end{array}$ & $\begin{array}{l}x x \\
v I I I \\
x \\
v \\
\end{array}$ & $\begin{array}{l}2 N 43 *, 0 C 604 A \\
T G 1-5 / 3 \square\end{array}$ & \\
\hline $\begin{array}{l}T R-15 / 2 \\
T V-15\end{array}$ & $\begin{array}{l}\text { TRI } \\
\text { THM }\end{array}$ & THY & XVIII & $T R-1-5 / 2 \square$ & \\
\hline
\end{tabular}




\begin{tabular}{|c|c|c|c|c|c|}
\hline \multicolumn{6}{|c|}{ GROUP I, NUMERICAL } \\
\hline IYPE XUMSER & $1 \times 0$ & IYPE & \begin{tabular}{|c} 
TABLE \\
NO.
\end{tabular} & SIMILAR TYPES & $\begin{array}{c}\text { COST SPCC. } \\
\text { NO. }\end{array}$ \\
\hline $\begin{array}{l}\text { VG15/5000 } \\
15465 \\
15 L I I \\
\text { OGTS16 } \\
\text { FEU } 16\end{array}$ & $\begin{array}{l}\text { OIO } \\
\text { PND } \\
\text { IC } \\
\text { REC } \\
\text { PMM }\end{array}$ & $\begin{array}{l}\operatorname{SIN} \\
\operatorname{SIN}\end{array}$ & $\begin{array}{l}11 \\
X I \\
X V I\end{array}$ & $\begin{array}{l}G G 1-C .5 / 5 a \\
\angle I-10 \\
02 P a\end{array}$ & \\
\hline $\begin{array}{l}G I-168 \\
G U 168 \\
L G-16 \\
M S-16 \\
P 16\end{array}$ & $\begin{array}{l}\text { TET } \\
\text { TRI } \\
\text { OIO } \\
\text { COU }\end{array}$ & $\begin{array}{l}\operatorname{SIN} \\
\operatorname{SIN} \\
\operatorname{SIN}\end{array}$ & $\begin{array}{l}111 \\
111 \\
x x 1 \\
x\end{array}$ & $\begin{array}{l}20250 \\
2 \times 55 *, 0<604 *\end{array}$ & \\
\hline $\begin{array}{l}P 16 A \\
P 168 \\
S G 16 P \\
T V-16 \\
V S-16\end{array}$ & $\begin{array}{l}\text { OIO } \\
\text { THM } \\
\text { COU }\end{array}$ & $\sin$ & $\begin{array}{l}x \\
x \\
v \\
x \vee I I I \\
x x I\end{array}$ & & \\
\hline $\begin{array}{l}\text { DGTS17 } \\
\text { FEU } 17 \\
\text { FEU-17A } \\
G-178 \\
G I-17\end{array}$ & $\begin{array}{l}\text { REC } \\
\text { PHM } \\
\text { PHM } \\
\text { TRI } \\
\text { TRI }\end{array}$ & $\begin{array}{l}\sin \\
\operatorname{SIn}\end{array}$ & $\begin{array}{l}X I \\
X V \\
X V I \\
\text { III } \\
I I I\end{array}$ & G4800 & \\
\hline $\begin{array}{r}\text { GU-17 } \\
L I-17 \\
45 T-17 \\
P 17 \\
P 174\end{array}$ & $\begin{array}{l}\text { BEA } \\
\text { IM } \\
\text { COU }\end{array}$ & TWN & $\begin{array}{l}x \\
\operatorname{lil} \\
\operatorname{VIII} \\
x \times I \\
x \\
x\end{array}$ & & \\
\hline $\begin{array}{r}P 178 \\
S G 17 S \\
F E U-18 \\
F E U-18 A \\
G I-18 B\end{array}$ & $\begin{array}{l}\text { DIO } \\
\text { PHM } \\
\text { PHM } \\
\text { TRI }\end{array}$ & $\sin$ & $\begin{array}{l}x \\
v \\
x v \\
x y I \\
I I I\end{array}$ & & \\
\hline $\begin{array}{l}\text { GU-18 } \\
G S-18 \\
\text { LI }-18 \\
P 18 \\
\text { P18A }\end{array}$ & $\begin{array}{l}\text { BEA } \\
\text { TRI } \\
\text { VI }\end{array}$ & $\begin{array}{l}\text { TWN } \\
\text { SIN }\end{array}$ & $\begin{array}{l}111 \\
v I I I \\
x \\
x\end{array}$ & $G K-20000$ & \\
\hline $\begin{array}{l}\text { P1BB } \\
\text { SG18S } \\
18 L K 2 B \\
18 L K 3 V \\
18 L K 4 B\end{array}$ & $\begin{array}{l}\text { OIO } \\
\text { TV } \\
\text { TV }\end{array}$ & $\sin$ & $\begin{array}{l}x \\
v \\
v I I I \\
\text { vIII } \\
\text { VIII }\end{array}$ & $70 P_{4} *$ & \\
\hline $\begin{array}{l}18 L K 5 B \\
18 L K 7 B \\
18 L K 15 \\
18 L M 35 \\
18 L M 35 V\end{array}$ & $\begin{array}{l}\text { TV } \\
\text { TV } \\
\text { TV } \\
\text { OS } \\
\text { OS }\end{array}$ & 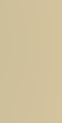 & $\begin{array}{l}\text { VIII } \\
\text { VIII } \\
\text { VIII } \\
\text { VIII } \\
\text { VIII }\end{array}$ & 7BP 7A* & \\
\hline $\begin{array}{l}18 \mathrm{LO} P \\
18 \mathrm{LO} 0 \mathrm{BB} \\
18 \mathrm{LO} 4 \mathrm{AA} \\
18 \mathrm{LO} 7 \mathrm{~V} \\
\text { FEU-19M }\end{array}$ & $\begin{array}{l}- \\
\text { TV } \\
\text { OD } \\
\text { OD } \\
\text { PHM }\end{array}$ & (1) & $\begin{array}{l}\text { VIII } \\
\text { VIII } \\
\text { VIII } \\
\text { VIII } \\
\text { xv }\end{array}$ & $\begin{array}{l}\text { 7BP 7A* } \\
7 J P P_{4}\end{array}$ & \\
\hline $\begin{array}{l}G 1-198 \\
\text { GU-19 } \\
P 19 \\
S G 195 \\
19 L \times 4 B\end{array}$ & $\begin{array}{l}\text { TRI } \\
\text { BEA } \\
\text { OIO } \\
\text { TV }\end{array}$ & $\begin{array}{l}\text { SIN } \\
\text { TWN } \\
\text { SIN }\end{array}$ & $\begin{array}{l}I 11 \\
I 11 \\
x \\
v \\
V I I I\end{array}$ & & \\
\hline $\begin{array}{l}F E U-20 \\
G \times 20 \\
I=20 / 1.5 \\
I F K 2 D \\
M-20 / 35\end{array}$ & $\begin{array}{l}\text { PMM } \\
\text { TRI } \\
\text { TRI } \\
\text { TRI }\end{array}$ & $\begin{array}{l}\text { SIN } \\
\text { THY } \\
\text { SIN }\end{array}$ & $\begin{array}{l}x y \\
\text { III } \\
V I 1 \\
x x\end{array}$ & GM-1AU & \\
\hline $\begin{array}{c}020 \\
P 20 \\
O V 20-P_{18} \\
T R-20 / 15 \\
V 20 / 20\end{array}$ & $\begin{array}{l}\text { TET } \\
\text { TRI } \\
\text { PIO }\end{array}$ & $\begin{array}{l}\text { SIN } \\
\text { SIN } \\
\text { THY } \\
\text { SIN }\end{array}$ & $x^{\ln }$ & $\begin{array}{l}\text { GM1-83* } \\
T R-1-6 / 15 \square \\
V 1-0.02 / 200\end{array}$ & \\
\hline $\begin{array}{l}\text { 20LMIYE } \\
\text { O21 } \\
\text { DGTS21 } \\
\text { GI-21B } \\
\text { GU21B }\end{array}$ & $\begin{array}{l}\text { PREC } \\
\text { REC } \\
\text { TRI } \\
\text { TRI }\end{array}$ & $\begin{array}{l}\sin \\
\operatorname{SIN}\end{array}$ & $\begin{array}{l}\text { VIII } \\
\text { XI } \\
X I \\
\text { III } \\
\text { III }\end{array}$ & 07A口 & \\
\hline $\begin{array}{l}\text { P21 } \\
\text { P21A }\end{array}$ & & & $\begin{array}{l}x \\
x\end{array}$ & & \\
\hline
\end{tabular}

\begin{tabular}{|c|c|c|c|c|c|}
\hline \multicolumn{6}{|c|}{ GROUP I. NUMERICAL } \\
\hline IYPE NUMBER & MINO & IYPE & $\begin{array}{l}\text { TABLE } \\
\text { No. }\end{array}$ & SIMILAR IYPES & $\begin{array}{l}\text { COST SPEC. } \\
\text { NO. }\end{array}$ \\
\hline OGTS22 & REC & & $x I$ & 0780 & \\
\hline FEU -22 & PHM & & $x v$ & & \\
\hline$G 1-22$ & TRI & $5 I N$ & III & & \\
\hline $\begin{array}{l}\text { GU22A } \\
\text { OGTS23 }\end{array}$ & $\begin{array}{l}\text { TRI } \\
\text { REC }\end{array}$ & $\operatorname{SIN}$ & $\begin{array}{ll}111 \\
X 1\end{array}$ & D7v口 & \\
\hline & & & & & \\
\hline $\begin{array}{l}\text { FEU-23 } \\
\text { GU23A }\end{array}$ & $\begin{array}{l}\text { PHM } \\
\text { TRI }\end{array}$ & $\sin$ & $\begin{array}{l}x y I \\
\text { III }\end{array}$ & & \\
\hline$G U-23 B$ & $T R I$ & $\operatorname{Sin}$ & II & & \\
\hline $23 L K_{1} 18$ & TV & & VIII & $9 C P_{4} *$ & \\
\hline $23 L \times 28$ & TV & & vili & & \\
\hline $23 L K 78$ & TV & & VIII & & \\
\hline $23 L K B B$ & TV & & vill & & \\
\hline $\begin{array}{l}23 L M 34 \\
23 L M 34 V\end{array}$ & $\begin{array}{l}\text { os } \\
\text { os }\end{array}$ & & $\begin{array}{l}\text { VIII } \\
\text { VIIII }\end{array}$ & $9 G P 7 *$ & \\
\hline $23 L 01 P$ & os & & VIII & 9GP7* & \\
\hline $23 \operatorname{LOS} 1 \mathrm{~A}$ & os & & VIII & & \\
\hline $\begin{array}{l}\text { DGTS24 } \\
\text { FEU-24 }\end{array}$ & $\begin{array}{l}\text { REC } \\
\text { PHM }\end{array}$ & & $\begin{array}{l}x I \\
x y\end{array}$ & 0760 & \\
\hline $\begin{array}{l}\text { GI-24A } \\
\text { GU24A }\end{array}$ & TR I & $\sin$ & III & & \\
\hline DGTSZS & REC & & $x 1$ & 0700 & \\
\hline FEU-25 & PHM & & $x v$ & & \\
\hline $61-25$ & $T R I$ & $51 N$ & III & & \\
\hline GU258 & TRI & $\sin$ & iil & & \\
\hline I5K25 & & & $x x$ & & \\
\hline P25 & & & $x$ & & \\
\hline P25A & & & $x$ & & \\
\hline $\begin{array}{l}P 258 \\
V K-25\end{array}$ & POW & & $\begin{array}{l}x \\
x 11\end{array}$ & & \\
\hline 25P1 & BEA & $\sin$ & ii & $25 L 6 *$ & \\
\hline $\begin{array}{r}25 P 15 \\
\text { OGTS26 }\end{array}$ & $\begin{array}{l}\text { BEA } \\
\text { REC }\end{array}$ & $\sin$ & $\begin{array}{l}11 \\
\times 1\end{array}$ & $25 L 6 *$ & \\
\hline FEU 260 & PHM & & $\begin{array}{l}x 1 \\
x \vee I\end{array}$ & & \\
\hline GU26A & TRI & $\operatorname{SIN}$ & III & & \\
\hline GU268 & TRI & $\sin$ & III & & \\
\hline P26 & & & $x$ & & \\
\hline P26A & & & $x$ & & \\
\hline P268 & & & $x$ & & \\
\hline $\begin{array}{l}\text { OGTS27 } \\
\text { FEU } 27\end{array}$ & $\begin{array}{l}\text { REC } \\
\text { PHM }\end{array}$ & & $\begin{array}{l}x 1 \\
x \vee I\end{array}$ & $072 \mathrm{HQ}$ & \\
\hline GU27A & TET & $\sin$ & 111 & & \\
\hline GU27B & TET & $\sin$ & 111 & B27-R* & \\
\hline P27A & & & $x$ & & \\
\hline GU28A & TET & $\sin$ & 111 & & \\
\hline GU28B & TET & $\sin$ & 111 & & \\
\hline M28 & TRI & $\sin$ & 111 & & \\
\hline $\begin{array}{r}\text { P28 } \\
\text { FEU-29 }\end{array}$ & PHM & & $\begin{array}{l}x \\
x y\end{array}$ & & \\
\hline 629 & TRI & $\sin$ & i11 & & \\
\hline $\begin{array}{r}\text { GU29 } \\
\text { P29 }\end{array}$ & TET & TWN & $x^{111}$ & $B 29-8 *$ & \\
\hline P29A & & & $\hat{x}$ & & \\
\hline $600-30$ & TRI & $\operatorname{SIN}$ & & $65-380$ & \\
\hline GI-30 & BEA & $T W N$ & 111 & 3E29* & \\
\hline GM I - 30 & TRI & $\sin$ & 111 & & \\
\hline $\begin{array}{l}\text { GS-30 } \\
\text { GU3OA }\end{array}$ & $\begin{array}{l}\text { COU } \\
\text { TRI }\end{array}$ & $\sin$ & $\begin{array}{l}x \times 1 \\
111\end{array}$ & & \\
\hline$M=30 / 450$ & TRI & $\operatorname{Sin}$ & & GMI-3nם & \\
\hline P30 & & & $x$ & & \\
\hline$V G-30$ & Pow & & $x[1$ & & \\
\hline $30 L \times 18$ & TV & & VIII & $31 L K 180$ & \\
\hline $30 \mathrm{Pl}$ & BEA & $\operatorname{SIN}$ & & 30 P $15 \mathrm{Sa}$ & \\
\hline $30 P 1 M$ & QBEA & $\operatorname{SIN}$ & 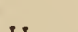 & 30pisa & \\
\hline $30 P 15$ & BEA & $\operatorname{SIN}$ & it & $30 P_{1 M}$ & \\
\hline 30TSIM & 010 & $\operatorname{SIN}$ & It & 30VKH1ם, 30T565a & $8078-56$ \\
\hline $\begin{array}{l}30 \text { TS65 } \\
30 V 01\end{array}$ & $\begin{array}{l}010 \\
010\end{array}$ & TWN & II & $30 V \times H 1 \square, 30 T 5149$ & $8078-56$ \\
\hline $\begin{array}{l}\text { 30VV1 } \\
\text { 30VKH1 }\end{array}$ & $\begin{array}{l}010 \\
010\end{array}$ & $\begin{array}{l}\operatorname{SIn} \\
\operatorname{SIN}\end{array}$ & II & $\begin{array}{l}\text { 30TS1Ma } \\
\text { 30TS6Sa }\end{array}$ & \\
\hline$F E U-31$ & PHM & & XVI & & \\
\hline GU31 & TET & SIN & III & & \\
\hline 31LK 18 & TV & & viil & & \\
\hline
\end{tabular}




\begin{tabular}{|c|c|c|c|c|c|}
\hline \multicolumn{6}{|c|}{ GROUP I, NUMERICAL } \\
\hline TYPE NUMBER & KiNO & TYPE & $\begin{array}{l}\text { TABLE } \\
\text { NO. }\end{array}$ & SIMILAR TYPES & $\begin{array}{c}\text { COST SPEC. } \\
\text { no. }\end{array}$ \\
\hline $31 L K 2 B$ & TV & & viII & 12 LP4* & \\
\hline $31 L M 32$ & os & & VIII & 12DP7A* & \\
\hline $31 L \mathrm{M} 32 \mathrm{~V}$ & os & & vili & & \\
\hline 31 LOIP & & & viit & $120 P 7 *$ & \\
\hline $31<033$ & os & & VIII & $12 G P 7 *$ & \\
\hline $\begin{array}{l}31 L 033 V \\
F E U-32\end{array}$ & $\begin{array}{l}\text { OS } \\
\text { PHM }\end{array}$ & & $\begin{array}{l}\text { VIII } \\
x y I\end{array}$ & & \\
\hline $\begin{array}{r}F E U-32 \\
G 32\end{array}$ & $\begin{array}{l}\text { PHM } \\
\text { TRI }\end{array}$ & $\operatorname{SIN}$ & $\begin{array}{l}x+1 \\
111\end{array}$ & & \\
\hline $\begin{array}{r}\text { GU32 } \\
\end{array}$ & BEA & TWN & 111 & $832 *$ & \\
\hline reutso & & & & & \\
\hline GU338 & TET & $\operatorname{SIN}$ & 111 & & \\
\hline $\begin{array}{l}\text { GU34B } \\
F E U-39\end{array}$ & $\begin{array}{l}\text { TET } \\
\text { PHM }\end{array}$ & $\sin$ & $\begin{array}{l}111 \\
x \vee I\end{array}$ & & \\
\hline GU-35B & TET & $\operatorname{SIN}$ & III & & \\
\hline 35 LK2 $2 B$ & TV & & viti & & $8815-58$ \\
\hline G36 & $T R I$ & SIN & III & & \\
\hline GK36 & $T R I$ & $\operatorname{Sin}$ & & $G K-20 \square$ & \\
\hline$G U-36 B$ & TET & SIN & III & & \\
\hline $\begin{array}{l}G U-37 B \\
G U-39 A\end{array}$ & $\begin{array}{l}\text { TR! } \\
\text { TET }\end{array}$ & $\begin{array}{l}\text { SIN } \\
\operatorname{SIN}\end{array}$ & $\begin{array}{l}111 \\
111\end{array}$ & & \\
\hline GU-39B & TET & SIN & III & & \\
\hline M39 & TRI & $\operatorname{SIN}$ & III & & \\
\hline FEU-4D & NSP & & $x \vee !$ & & \\
\hline $\begin{array}{l}G U-40 B \\
V 4 D / 1 D D\end{array}$ & $\begin{array}{l}\text { TET } \\
\text { DIO }\end{array}$ & $\begin{array}{l}\sin \\
\operatorname{SIN}\end{array}$ & III & $V 1-D+1 / 4 D \square$ & \\
\hline $4 D L K 1 B$ & TV & & vint & 16AP4* & \\
\hline FEU -42 & NSP & & $x \mathrm{VI}$ & & \\
\hline$K 42$ & KLY & & ix & & \\
\hline $\begin{array}{l}\text { FEU } 42 \text { LM2YE } \\
\text { FEU } 43\end{array}$ & NSP & & $\begin{array}{l}\text { vIII } \\
x \vee I\end{array}$ & & \\
\hline $43 L K 2 B$ & TV & & VIII & & $8814-58$ \\
\hline $43 L K 38$ & TV & & VIII & & \\
\hline $43 L K_{6} 6 B$ & TV & & VIII & & \\
\hline $43 L K>B$ & TV & & vili & & \\
\hline $43 L K 8 B$ & TV & & vitt & & \\
\hline FEU-44 & NSP & & $x \vee I$ & & \\
\hline$F E U-45$ & NSP & & $x V I$ & & \\
\hline 45LM1B & & & VIII & & \\
\hline$F E U-46$ & NSP & & $x \vee I$ & & \\
\hline 646 & TRI & $\operatorname{SIN}$ & III & & \\
\hline FEU -47 & NSP & & $x \vee !$ & & \\
\hline 647 & TRI & $\operatorname{SIN}$ & III & & \\
\hline $58-47$ & PND & SIN & II & & \\
\hline$F E U-48$ & NSP & & $x V_{1}$ & & \\
\hline$\times 48$ & KLY & & Ix & & \\
\hline FEU-49 & PHM & & $x \vee I$ & & \\
\hline$G-49$ & TRI & $\operatorname{SIN}$ & & $69-4 \square$ & \\
\hline GD-5D & $T R I$ & $\operatorname{SIN}$ & & $G-46 \square$ & \\
\hline $\begin{array}{l}\text { GU5D } \\
I-50 / 1.5\end{array}$ & PND & $\sin$ & III & L\$50" & \\
\hline $1-50 / 1 \cdot 5$ & TRI & THY & VII & & \\
\hline IFK50 & & & $x x$ & & \\
\hline L.S5D & PPND & $\operatorname{SIN}$ & & GU5DA & \\
\hline $\begin{array}{r}\text { M50 } \\
Y G-50\end{array}$ & TRI & $\operatorname{SIN}$ & 111 & & \\
\hline $\begin{array}{l}V G=50 \\
V K=50\end{array}$ & $\begin{array}{l}\text { Pow } \\
\text { pow }\end{array}$ & & $\begin{array}{l}x 11 \\
\times 11\end{array}$ & & \\
\hline GMS1A & TRI & $\operatorname{SIN}$ & 111 & & \\
\hline$S B-51$ & PND & $\sin$ & il & & \\
\hline STSV5i & PHO & & $x v$ & & \\
\hline FEU -52 & PHM & & $x \vee I$ & & \\
\hline FEU -53 & PHM & & $x v i$ & & \\
\hline M53 & TRI & $\operatorname{SIN}$ & III & & \\
\hline $\begin{array}{l}53 L K 2 B \\
53 L K 3 B\end{array}$ & $\begin{array}{l}T V \\
T V\end{array}$ & & $\begin{array}{l}\text { VIII } \\
\text { VIII }\end{array}$ & & \\
\hline 53 LK4 TS & & & VIII & & \\
\hline 53 LK58 & TV & & VIII & & \\
\hline $\begin{array}{l}G-54 \\
R-54\end{array}$ & TRI & $\sin$ & $x \times I 1$ & $G S-6 \square$ & \\
\hline$G-56$ & TRI & $\sin$ & & G29ם & \\
\hline $\begin{array}{l}\text { GM5 } 7 \\
\text { M57 }\end{array}$ & $\begin{array}{l}\text { TRI } \\
\text { TRI }\end{array}$ & SIN & II & MS5DE口, M457ם, UB1BD" & \\
\hline & & & & & \\
\hline $\begin{array}{r}50-57 \\
G-5 B\end{array}$ & $\begin{array}{l}\text { PND } \\
\text { TRI }\end{array}$ & $\begin{array}{l}\operatorname{SIN} \\
\operatorname{SIN}\end{array}$ & II & GK-3000D & \\
\hline
\end{tabular}

\begin{tabular}{|c|c|c|c|c|c|}
\hline \multicolumn{6}{|c|}{ GROUP I, NUMERICAL } \\
\hline TYPE NUMBER & KINO & TYPE & $\begin{array}{l}\text { TABLE } \\
\text { HO. }\end{array}$ & SIMILAR TYPES & $\begin{array}{l}\text { COST SPEC. } \\
\text { NO. }\end{array}$ \\
\hline $\begin{array}{r}G M 60 \\
35-6 D \\
G 61 \\
G 62 \\
G-64\end{array}$ & $\begin{array}{l}\text { TRI } \\
\text { COU } \\
\text { TRI } \\
\text { TRI } \\
\text { TRI }\end{array}$ & $\begin{array}{l}\operatorname{SIN} \\
\operatorname{SIN} \\
\operatorname{SIN} \\
\operatorname{SIN}\end{array}$ & $\begin{array}{l}\text { III } \\
x \times 1 \\
\text { III } \\
\text { III }\end{array}$ & $G \varsigma-3 B a$ & \\
\hline $\begin{array}{l}665 \\
\text { G68 } \\
\text { GI }-7 D B \\
\text { GM-7D } \\
\text { GMTDB }\end{array}$ & $\begin{array}{l}\text { TRI } \\
\text { TRI } \\
\text { TRI } \\
\text { TRI } \\
\text { TRI }\end{array}$ & $\begin{array}{l}\operatorname{SIN} \\
\operatorname{SIN} \\
\operatorname{SIN} \\
\operatorname{SIN} \\
\operatorname{SIN}\end{array}$ & $\begin{array}{l}\text { III } \\
\text { III } \\
\text { III } \\
\text { III } \\
\text { III }\end{array}$ & & \\
\hline $\begin{array}{l}\text { ISP7D } \\
\text { LD70 } \\
\text { V7D/10DD } \\
\text { GK71 } \\
\text { GU72 }\end{array}$ & $\begin{array}{l}\text { TRI } \\
\text { OIO } \\
\text { PND } \\
\text { PND }\end{array}$ & $\begin{array}{l}\operatorname{SIN} \\
\operatorname{SIN} \\
\operatorname{SIN} \\
\operatorname{SIN}\end{array}$ & $\begin{array}{l}x x \\
111 \\
111\end{array}$ & $\begin{array}{l}G 1-70 B \square \\
V 1-D \cdot 3 / 7 D \square \\
G 471 \square\end{array}$ & \\
\hline $\begin{array}{l}M 74 \\
7555-3 D \\
G 1-768 \\
\text { GUBO } \\
M 80\end{array}$ & $\begin{array}{r}\text { TRI } \\
\text { POIO } \\
\text { TRI } \\
\text { PND } \\
\text { TRI }\end{array}$ & $\begin{array}{l}\operatorname{SIN} \\
\operatorname{SIN} \\
\operatorname{SIN} \\
\operatorname{SIN} \\
\operatorname{SIN}\end{array}$ & $\begin{array}{l}111 \\
111 \\
111 \\
111\end{array}$ & $\begin{array}{l}\text { SG2SE, OA3* } \\
\text { OS450*, P8DOC口 }\end{array}$ & \\
\hline $\begin{array}{r}\text { GUB I } \\
\text { GMI-83 } \\
\text { GB } \\
V 0-88 \\
\text { GMI-89 }\end{array}$ & $\begin{array}{l}\text { PND } \\
\text { TET } \\
\text { TRI } \\
\text { DIO } \\
\text { TET }\end{array}$ & $\begin{array}{l}\text { SIN } \\
\text { SIN } \\
\text { SIN } \\
\text { TWN } \\
\text { SIN }\end{array}$ & $\begin{array}{l}1 ! 1 \\
111 \\
111 \\
111\end{array}$ & $\begin{array}{l}\text { QV20-P18* } \\
\text { 4VKHID } \\
\text { G-4890D }\end{array}$ & \\
\hline $\begin{array}{c}\text { GUB 9A } \\
\text { GU89B } \\
\text { M89 } \\
\text { GMI-9D } \\
\text { GS90B }\end{array}$ & $\begin{array}{l}\text { TRI } \\
\text { TRI } \\
\text { TRI } \\
\text { TET } \\
\text { TRI }\end{array}$ & $\begin{array}{l}\operatorname{SIN} \\
\operatorname{SIN} \\
\operatorname{SIN} \\
\operatorname{SIN} \\
\operatorname{SIN}\end{array}$ & $\begin{array}{l}111 \\
111 \\
111 \\
111 \\
111\end{array}$ & $\begin{array}{l}\text { B89A* } \\
\text { BB9R-A* } \\
\text { G-490\%A }\end{array}$ & \\
\hline $\begin{array}{r}\text { LD-90 } \\
\text { MTKH9D } \\
\text { RB-90 }\end{array}$ & $\begin{array}{l}\text { TRI } \\
\text { TRI }\end{array}$ & $\begin{array}{l}\text { SIN } \\
\text { THY }\end{array}$ & $\begin{array}{l}v \llbracket 1 \\
x \times I 1\end{array}$ & GS-90Bם & \\
\hline $\begin{array}{c}T G I-9 D / 8 \\
691\end{array}$ & $\begin{array}{l}\text { TRI } \\
\text { TRI }\end{array}$ & $\begin{array}{l}\text { THY } \\
\text { SIN }\end{array}$ & 111 & TGI-1-90/8 & \\
\hline $\begin{array}{c}G-92 \\
L-99 \\
G=100 \\
G=100 A \\
G D-10 D\end{array}$ & $\begin{array}{l}\text { TRI } \\
\text { PTG } \\
\text { TRI } \\
\text { TRI } \\
\text { TRI }\end{array}$ & $\begin{array}{l}\operatorname{SIN} \\
\operatorname{SIN} \\
\operatorname{SIN} \\
\operatorname{SIN} \\
\operatorname{SIN}\end{array}$ & & 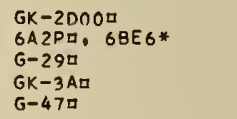 & \\
\hline $\begin{array}{l}\text { GKE } 10 D \\
\text { GM } 10 D \\
1-10 D / 1 \cdot 0 \\
1-10 D / 5 . D \\
\text { I SSHIDD- }\end{array}$ & $\begin{array}{l}\text { TET } \\
\text { TRI } \\
\text { TRI } \\
\text { TRI }\end{array}$ & $\begin{array}{l}\text { SIN } \\
\text { SIN } \\
\text { THY } \\
\text { THY }\end{array}$ & $\begin{array}{l}1 I 1 \\
111 \\
V I I \\
V I I \\
x x\end{array}$ & $G E-1 \#$ & \\
\hline $\begin{array}{l}\text { ISSHIDD-3 } \\
\text { LIDO } \\
V G-10 D \\
V K-1 D D \\
0101\end{array}$ & $\begin{array}{l}\text { PPND } \\
\text { POW } \\
\text { POW } \\
\text { REC }\end{array}$ & D10 & $\begin{array}{l}x \times \\
x 11 \\
x 11 \\
x 11 \\
x !\end{array}$ & $6 B 2 \mathrm{PA}$ & \\
\hline $\begin{array}{l}\text { DIOIA } \\
\text { LI-101 } \\
\text { P101 } \\
\text { P101A } \\
\text { D102 }\end{array}$ & $\begin{array}{l}\text { REC } \\
\text { IC }\end{array}$ & & $\begin{array}{l}x ! \\
v ! I I \\
x \\
x \\
x !\end{array}$ & & \\
\hline $\begin{array}{l}\text { DIn2A } \\
\text { P1D2 } \\
\text { D1D3 } \\
D 103 A \\
\text { P103 }\end{array}$ & $\begin{array}{l}\text { REC } \\
\text { REC }\end{array}$ & & $\begin{array}{l}x I \\
x \\
x 1 \\
x 1 \\
x\end{array}$ & & \\
\hline $\begin{array}{l}S-103 \\
D 104 \\
0104 A \\
L-104 \\
P 104\end{array}$ & $\begin{array}{l}\text { TET } \\
\text { REC } \\
\text { REC } \\
\text { PND }\end{array}$ & $\begin{array}{l}\operatorname{SIN} \\
\operatorname{SIN}\end{array}$ & $\begin{array}{l}x ! \\
x ! \\
x\end{array}$ & 6K4PD, GBAG* & \\
\hline $\begin{array}{l}\text { D105 } \\
\text { D105A } \\
\text { P105 }\end{array}$ & $\begin{array}{l}\text { REC } \\
\text { REC }\end{array}$ & & $\begin{array}{l}x 1 \\
x 1 \\
x\end{array}$ & & \\
\hline $\begin{array}{l}10555-30 \\
0106\end{array}$ & $\begin{array}{l}\text { DIO } \\
\text { REC }\end{array}$ & $\operatorname{SIN}$ & $\times 1$ & SG35口, OC3* & \\
\hline $\begin{array}{l}\text { D106A } \\
\text { P106 }\end{array}$ & REC & & $\begin{array}{l}x 1 \\
x\end{array}$ & & \\
\hline
\end{tabular}




\begin{tabular}{|c|c|c|c|c|c|}
\hline \multicolumn{6}{|c|}{ GROUP I, NUMERICAL } \\
\hline TYPE NUMBER & KIMO & TYPE & $\begin{array}{l}\text { TABLE } \\
\text { NO. }\end{array}$ & SIMILAR TYPES & $\begin{array}{c}\text { COST SPEC. } \\
\text { HO. }\end{array}$ \\
\hline $\begin{array}{l}T G I-325 / 16 \\
I=1-350 / 0.8 \\
R-350 \\
R 8-350 \\
V 0-360\end{array}$ & $\begin{array}{l}\text { TRI } \\
\text { TRI } \\
\\
010\end{array}$ & $\begin{array}{l}\text { THY } \\
\text { THY } \\
\text { SIN }\end{array}$ & $\begin{array}{l}v I I \\
x \times I I \\
x \times I I \\
\text { IV }\end{array}$ & MTI $-5 \square, T G I-1-325 / 16 \square$ & \\
\hline $60-400$ & TRI & $\operatorname{SIN}$ & & $G \varsigma-6 \square$ & \\
\hline $\begin{array}{l}M 400 \\
T G-400 / 15\end{array}$ & $\begin{array}{l}\text { TRI } \\
\text { TRI }\end{array}$ & $\begin{array}{l}\text { SIN } \\
\text { THY }\end{array}$ & I I I & TR1-130/15ם & \\
\hline$T G 1400 / 3.5$ & TRI & THY & & $T G I-2-400 / 3.5 \square$ & \\
\hline 0401 & MOO & & XIII & & \\
\hline LI $1-401$ & & & VIII & & \\
\hline $\begin{array}{l}M 401 \\
P 401\end{array}$ & TRI & $\sin$ & $x^{111}$ & 2N112* & \\
\hline P402 & & & & $58-10 n *$ & \\
\hline $0403 A$ & $\operatorname{MIX}$ & & XIII & & \\
\hline 04038 & MIX & & & & \\
\hline $\begin{array}{l}04 n 3 V \\
P 4 n 3\end{array}$ & MIX & & $\begin{array}{l}x \ln 1 \\
x\end{array}$ & OC614* & \\
\hline P403A & & & $x$ & $0<614 *$ & \\
\hline P404 & & & $x$ & & \\
\hline P404A & & & $x$ & & \\
\hline 0405 & OET & & $\begin{array}{llll}x & 1 & 1 & 1 \\
x\end{array}$ & & \\
\hline $0405 \mathrm{~A}$ & OET & & $x+111$ & & \\
\hline $\begin{array}{l}0405 \mathrm{AP} \\
0405 \mathrm{~B}\end{array}$ & $\begin{array}{l}O E T \\
\text { OET }\end{array}$ & & 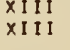 & & \\
\hline $04058 P$ & DET & & XIII & & \\
\hline $\begin{array}{l}\text { P405 } \\
\text { P405A }\end{array}$ & & & $\begin{array}{l}x \\
x \\
x\end{array}$ & & \\
\hline P406 & & & $x$ & GT-60* & \\
\hline P407 & & & $x$ & & \\
\hline $\begin{array}{l}\text { P408 } \\
P 409\end{array}$ & & & $x$ & & \\
\hline$T-409$ & 010 & IGM & IV & & \\
\hline $\begin{array}{l}6410 \\
\text { P410 }\end{array}$ & TRI & $\sin$ & If I & & \\
\hline P410A & & & $x$ & & \\
\hline $\begin{array}{c}P 410 A \\
T-410\end{array}$ & & & $x$ & & \\
\hline $410 R$ & $\begin{array}{l}\text { OIO } \\
\text { KLY }\end{array}$ & IGM & $\begin{array}{l}\text { IV } \\
\text { Ix }\end{array}$ & & \\
\hline G411 & PNO & $\sin$ & II & $\mathrm{K} 2 \mathrm{H} 1 \mathrm{PO}$ & \\
\hline P411 & & & $x$ & AF 114" & \\
\hline P411A & & & $x$ & AF $114 *$ & \\
\hline$T-411$ & 010 & IGM & IV & & \\
\hline $\begin{array}{l}6412 \\
6413\end{array}$ & $\begin{array}{l}\text { PNO } \\
\text { PNOO }\end{array}$ & $\sin$ & $\begin{array}{l}\text { III } \\
\text { I I I }\end{array}$ & GLH2P口 & \\
\hline $\begin{array}{l}0413 \\
6414\end{array}$ & $\begin{array}{l}\text { PNO } \\
\text { PNO }\end{array}$ & $\begin{array}{l}\sin \\
\operatorname{Sin}\end{array}$ & 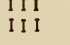 & & \\
\hline P414 & & & $x$ & & \\
\hline P414A & & & $x$ & & \\
\hline $\begin{array}{l}\text { P414B } \\
\text { P415 }\end{array}$ & & & $\begin{array}{l}x \\
x\end{array}$ & & \\
\hline $\begin{array}{l}\text { P415 } \\
\text { P415A }\end{array}$ & & & $\hat{x}$ & & \\
\hline P415B & & & $x$ & & \\
\hline P416 & & & $x$ & & \\
\hline P416A & & & $\begin{array}{l}x \\
x \\
x\end{array}$ & & \\
\hline $\begin{array}{l}P 4168 \\
P 416 V\end{array}$ & & & $\begin{array}{l}x \\
x\end{array}$ & & \\
\hline 6417 & TRI & $\operatorname{SIN}$ & III & & \\
\hline 6418 & PNO & $\sin$ & iil & & \\
\hline 6422 & PND & $\operatorname{SIN}$ & III & & \\
\hline $\begin{array}{l}6424 \\
6425\end{array}$ & $\begin{array}{l}\text { PND } \\
\text { PNO }\end{array}$ & $\begin{array}{l}\operatorname{SIN} \\
\text { SIN }\end{array}$ & $\begin{array}{ll}\text { I I I } \\
\text { I I I }\end{array}$ & & \\
\hline 6430 & TR I & $\operatorname{SIN}$ & II I & & \\
\hline$R 8-430$ & $1 \times$ & siv & $x \times 11$ & & \\
\hline G431 & TRI & SIN & I I I & $\begin{array}{l}\text { G431AD } \\
\text { G431 }\end{array}$ & \\
\hline $\begin{array}{r}\text { G431A } \\
G-431 R\end{array}$ & $\begin{array}{l}\text { TRI } \\
\text { TRI }\end{array}$ & $\begin{array}{l}\operatorname{SIN} \\
\operatorname{SIN}\end{array}$ & I11 & $\begin{array}{l}G 431 \\
G S-400\end{array}$ & \\
\hline 6433 & TRI & $\sin$ & 111 & G433A口 & \\
\hline G433A & TRI & $\sin$ & III & 6433 & \\
\hline $\begin{array}{l}M 435 \\
G 441\end{array}$ & $\begin{array}{l}\text { TRI } \\
\text { TRI }\end{array}$ & $\begin{array}{l}\operatorname{SIN} \\
\operatorname{SIN}\end{array}$ & $\begin{array}{l}\text { III } \\
\text { III }\end{array}$ & & \\
\hline $\begin{array}{r}6441 \\
G-450\end{array}$ & TR1 & $\begin{array}{l}\sin \\
\sin \end{array}$ & M & Gx-20กnם & \\
\hline $\begin{array}{l}05450 \\
R-450\end{array}$ & PNO & $\sin$ & $x \times I I$ & GU80\%, & \\
\hline
\end{tabular}

\begin{tabular}{|c|c|c|c|c|c|}
\hline \multicolumn{6}{|c|}{ GROUP I, NUMERICAL } \\
\hline TYPE NUMBER & Kino & TYPE & $\begin{array}{l}\text { TABLE } \\
\text { HO. }\end{array}$ & SIMILAR TYPES & $\begin{array}{c}\text { COST SPEC. } \\
\text { NO. }\end{array}$ \\
\hline$G=450$ & TRI & $\operatorname{SIN}$ & III & & \\
\hline$M-451$ & TRI & $\sin$ & & $G M-51 A D$ & \\
\hline$G-452$ & TRI & $\sin$ & I I I & G-431AD & \\
\hline $\begin{array}{l}G-454 \\
M 457\end{array}$ & $\begin{array}{l}\text { TRI } \\
\text { PTRI }\end{array}$ & $\begin{array}{l}\operatorname{SIN} \\
\operatorname{SIN}\end{array}$ & III & 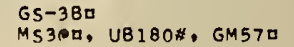 & \\
\hline$M-470$ & $T R I$ & $\operatorname{SIN}$ & & $6 M-70 \square$ & \\
\hline 6471 & PPNO & SIN & & GK>10 & \\
\hline 6472 & $T R I$ & $\operatorname{SIN}$ & III & & \\
\hline $\begin{array}{r}G 480 \\
G-483\end{array}$ & $\begin{array}{ll}\text { PTRI } \\
\text { PTET }\end{array}$ & $\sin$ & & $\begin{array}{l}G I-170 \square \\
G M I-83 \square\end{array}$ & \\
\hline$G-483$ & TET & $\sin$ & & GMI-83ם & \\
\hline $\begin{array}{r}G 484 \\
G-489\end{array}$ & $\begin{array}{l}\text { TRI } \\
\text { PTET }\end{array}$ & $\begin{array}{l}\text { SIN } \\
\text { SIN }\end{array}$ & I I I & GMI -890 & \\
\hline $6-490$ & PTET & $\operatorname{SIN}$ & & GMI-9nen & \\
\hline IFK5nO & & & $x x$ & & \\
\hline IFP500 & & & $x x$ & & \\
\hline I SSH5OO & & & $x x$ & & \\
\hline VGV 500 & POW & & $x 11$ & & \\
\hline P501 & & & $x$ & & \\
\hline $\begin{array}{l}\mathrm{PSO1A} \\
\mathrm{PSO2}\end{array}$ & & & $\begin{array}{l}x \\
x\end{array}$ & & \\
\hline P5O2A & & & $x$ & & \\
\hline $\mathrm{PSO} 28$ & & & $\hat{x}$ & & \\
\hline $\mathrm{P} 502 \mathrm{~V}$ & & & $\mathrm{x}$ & & \\
\hline $\begin{array}{l}\mathrm{P5O3} \\
\mathrm{P5O3A}\end{array}$ & & & $\begin{array}{l}x \\
x\end{array}$ & & \\
\hline M6nO & TR I & $\sin$ & & GM60口 & \\
\hline Pon1 & (बारा & $\sin$ & $x$ & GM600 & \\
\hline PGOIA & & & $\hat{x}$ & & \\
\hline $\begin{array}{l}P 6018 \\
0602 A\end{array}$ & vio & & $\begin{array}{l}x \\
x I I I\end{array}$ & & \\
\hline & & & & & \\
\hline $\begin{array}{l}06028 \\
0602 V\end{array}$ & $\begin{array}{ll}V I O \\
\text { VET }\end{array}$ & & $\begin{array}{lll}X I 1 \\
\end{array}$ & 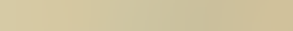 & \\
\hline $\mathrm{P}_{602}$ & OET & & $x^{x 111}$ & & \\
\hline$P 6 \cap 2 A$ & & & & & \\
\hline 0603 & v10 & & $X I I I$ & & \\
\hline P6n4 & & & $x$ & & \\
\hline P604A & & & $\begin{array}{l}x \\
x\end{array}$ & & \\
\hline $\begin{array}{l}P 6 \cap 4 \mathrm{~B} \\
700 \mathrm{AO}\end{array}$ & MAG & & $\begin{array}{l}X \\
I X\end{array}$ & & \\
\hline $706 \mathrm{AU}$ & MAG & & Ix & & \\
\hline $707 \mathrm{~A} / \mathrm{B}$ & $K L Y$ & & Ix & & \\
\hline $\begin{array}{l}714 \mathrm{AU} \\
\mathrm{LK}-715\end{array}$ & $\begin{array}{l}\text { MAG } \\
\text { OTV }\end{array}$ & & Ix & $18 L K 15 \square$ & \\
\hline 720AYE & MAG & & IX & & \\
\hline $723 \mathrm{~A} / \mathrm{B}$ & $K L Y$ & & Ix & & \\
\hline $725 A$ & MAG & & $1 x$ & & \\
\hline LK $=726$ & TV & & $t x$ & $18 L K 38 \square$ & \\
\hline $\begin{array}{r}726 \\
L 0-729\end{array}$ & $\begin{array}{l}K L Y \\
0 S\end{array}$ & & $I x$ & 8LO29R * 38R1A* & \\
\hline LO-730 & os & & & 8LO30口 & \\
\hline L0-731 & os & & & 13 LM31ם & \\
\hline LO-732 & os & & & 31 LM 32 & \\
\hline L0-733 & os & & & $31 \operatorname{LO} 33 \mathrm{D}$ & \\
\hline $\begin{array}{l}\text { LO-734 } \\
\text { LO-735 }\end{array}$ & $\begin{array}{l}\text { os } \\
\text { os }\end{array}$ & & & $\begin{array}{l}\text { 23LM34R } \\
18 L \text { L } 35 \square\end{array}$ & \\
\hline LO-736 & - os & & & $13 L 036 \square$ & \\
\hline LO-737 & - os & & & $131037 \square$ & \\
\hline LO-738 & os & & & $5 L 038 \square, 2 A P_{1} *$ & \\
\hline $\begin{array}{r}\text { Lo-739 } \\
\text { LK } 740\end{array}$ & $\begin{array}{l}\text { Os } \\
\text { TV }\end{array}$ & & & $\begin{array}{l}\text { 8LO39D } \\
18 L 04080,1 J R 4 \pi\end{array}$ & \\
\hline$L 0-743$ & - 00 & & & $10 L 0430$ & \\
\hline L0747 & 00 & & & $18 L 0470$ & \\
\hline $10-748$ & 00 & & & $18 L 0480$ & \\
\hline $\begin{array}{l}\begin{array}{l}10749 \\
\text { GK } 750\end{array}\end{array}$ & $\begin{array}{l}\text { OS } \\
\text { TRI }\end{array}$ & $\sin$ & III & $13 L 049 \square$ & $1709=55$ \\
\hline L0751 & os & & & $23 L 0510$ & \\
\hline $\begin{array}{l}\text { LOT54 } \\
\text { M800 }\end{array}$ & - os & & & 13L054ロ & \\
\hline $\begin{array}{l}\text { M800 } \\
P 800\end{array}$ & $\begin{array}{r}T R ! \\
\text { PNO }\end{array}$ & $\begin{array}{l}\operatorname{SIN} \\
\operatorname{SIN}\end{array}$ & 111 & GU80\%म, US450" & \\
\hline Gon7 & BEA & $\sin$ & III & $8 \cap 7 *$ & $838 v-57$ \\
\hline 0808 & REG & & $x \pm 1$ & & \\
\hline 0809 & REG & & $x I I$ & & \\
\hline
\end{tabular}




\begin{tabular}{|c|c|c|c|c|c|}
\hline \multicolumn{6}{|c|}{ GROUP I, NUMERICAL } \\
\hline TYPE NUMBER & RINO & TYPE & $\begin{array}{c}\text { TABLE } \\
\text { Yo. }\end{array}$ & SIMILAR TYPES & COSI SPEC. \\
\hline 0810 & REG & & $\times 11$ & & \\
\hline 0811 & REG & & $\times 11$ & $811-A=$ & \\
\hline 6811 & TRI & SIN & 111 & & \\
\hline 0813 & REG & & $\times 11$ & & \\
\hline$G-813$ & $B E A$ & SIN & & $G U-130,813 *$ & \\
\hline$G-827$ & TET & SIN & & $G U-278 \square, 827 K *$ & \\
\hline$G-829$ & TET & TWN & & $G U-29 \square, 829-8 *$ & \\
\hline$G=832$ & BEA & TWN & & $G U-32 a, 832 A$ & \\
\hline $\begin{array}{r}G 837 \\
G-880\end{array}$ & $\begin{array}{l}\text { PNO } \\
\text { TRI }\end{array}$ & $\begin{array}{l}\text { SIN } \\
\text { TWN }\end{array}$ & III & $\begin{array}{l}0512 / 500 * 837 * \\
G U-12 A \mathrm{~A}, 880^{*}\end{array}$ & \\
\hline G889 & $T R I$ & $\operatorname{SIN}$ & III & $889-A *$ & \\
\hline$T G-884$ & TRI & THY & & $\mathrm{TGl}-0.1 / 0.30,884 *$ & \\
\hline 6891 & TRI & SIN & III & $891 *$ & \\
\hline 601000 & TRI & SIN & & $G-29 \square$ & \\
\hline GKEI0OO & TET & $\operatorname{SIN}$ & III & & \\
\hline $\mathrm{M}=1000$ & TRI & SIN & & GM- $20 \cap 0$ & \\
\hline VGVInO0 & POW & & $x I^{x}$ & & \\
\hline $\begin{array}{l}\text { VKVI000 } \\
01001\end{array}$ & $\begin{array}{l}\text { POW } \\
\text { REC }\end{array}$ & & $\begin{array}{l}x I I \\
x I\end{array}$ & & \\
\hline D1001A & REC & & $x I$ & & \\
\hline 01002 & REC & & $x I$ & & \\
\hline $01002 \mathrm{~A}$ & REC & & $x 1$ & & \\
\hline $01003 A$ & REC & & $x I$ & & \\
\hline $\begin{array}{l}\text { TG1050 } \\
\text { IFP1500 }\end{array}$ & TRI & THY & $x x$ & $T G 2-0.1 / 0.12$ & \\
\hline $\begin{array}{l}1502 \\
1504\end{array}$ & 010 & $\operatorname{SIN}$ & & 5TS9S & \\
\hline 1506 & BRI & $\begin{array}{l}\text { SIN } \\
\text { TWN }\end{array}$ & II & & \\
\hline 1509 & BEA & TWN & II & & \\
\hline 1511 & PNO & SIN & 11 & & \\
\hline 1512 & PNO & $\operatorname{SIN}$ & 11 & & \\
\hline 1514 & PNO & SIN & 11 & & \\
\hline G1625 & $8 E A$ & $\operatorname{SIN}$ & III & $1625 *$ & \\
\hline $\begin{array}{l}\text { GK2000 } \\
\text { IFK2000 }\end{array}$ & TRI & $\operatorname{SIN}$ & $\operatorname{lII}_{x x}$ & & \\
\hline TG2050 & $T E T$ & THY & & $T G 1-0.1 / 1.30,2050 *$ & \\
\hline GK3000 & TRI & SIN & III & & $7780-55$ \\
\hline $\begin{array}{l}M-3000 \\
P I=3000\end{array}$ & TRI & SIN & & GMI I - IB口 & \\
\hline $\begin{array}{l}P I-3000 \\
G I-3100\end{array}$ & PPNO & SIN & III & GI-80ם & \\
\hline$G I-3100$ & TRI & SIN & $\Pi$ & & \\
\hline IFP $4 \cap 00$ & & & $x x$ & & \\
\hline 4671 & PTRI & $\operatorname{SIN}$ & & $6517 \mathrm{Ha}$ & \\
\hline G-50no & TRI & TWN & & $65=38 a$ & \\
\hline $\begin{array}{l}\text { IFP } 15000 \\
\text { IFK20000 }\end{array}$ & & & $\begin{array}{l}x x \\
x x\end{array}$ & & \\
\hline $\begin{array}{l}\text { G40011 } \\
\text { IFK80000 }\end{array}$ & TRI & SIN & $\begin{array}{l}111 \\
x \times\end{array}$ & & \\
\hline
\end{tabular}




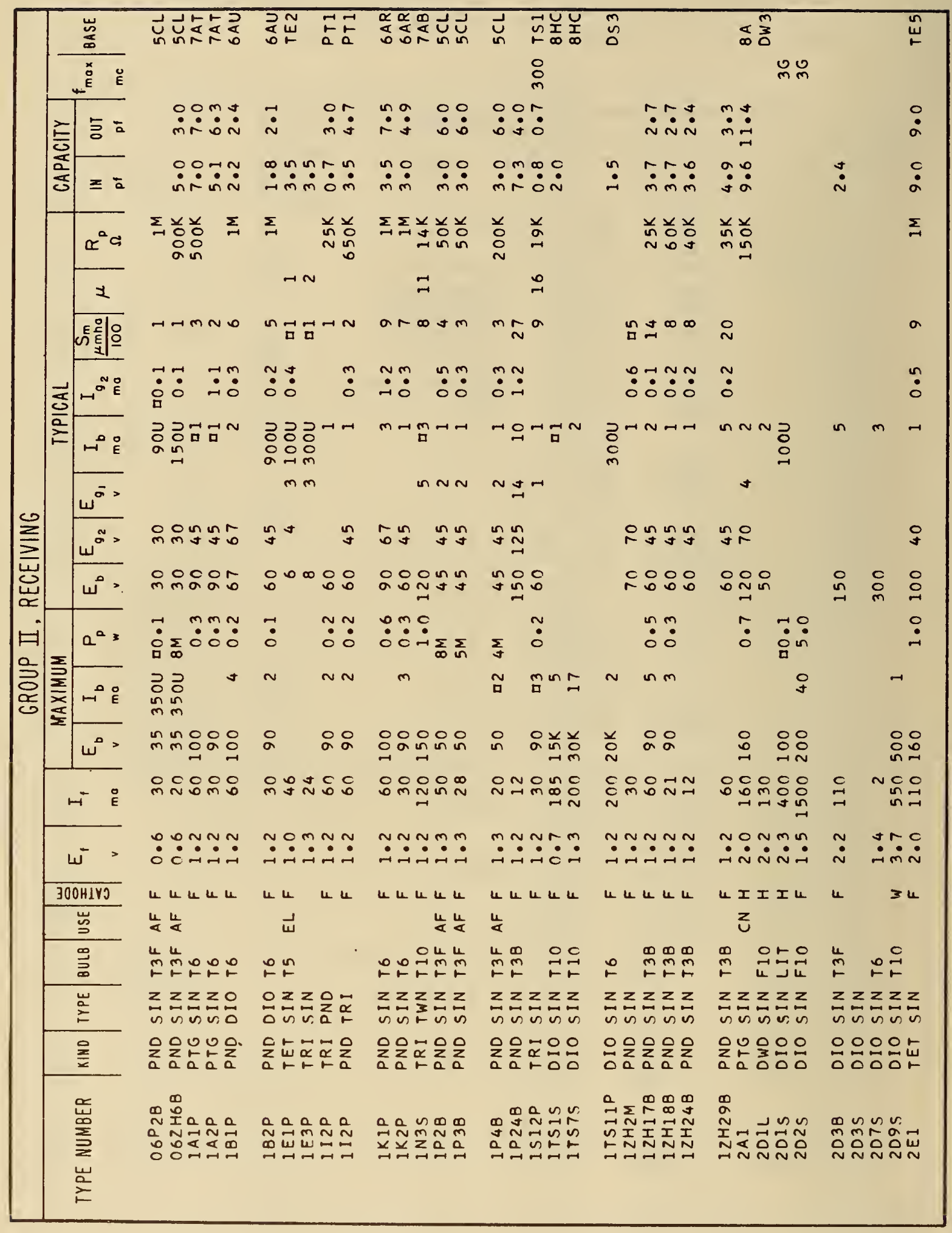




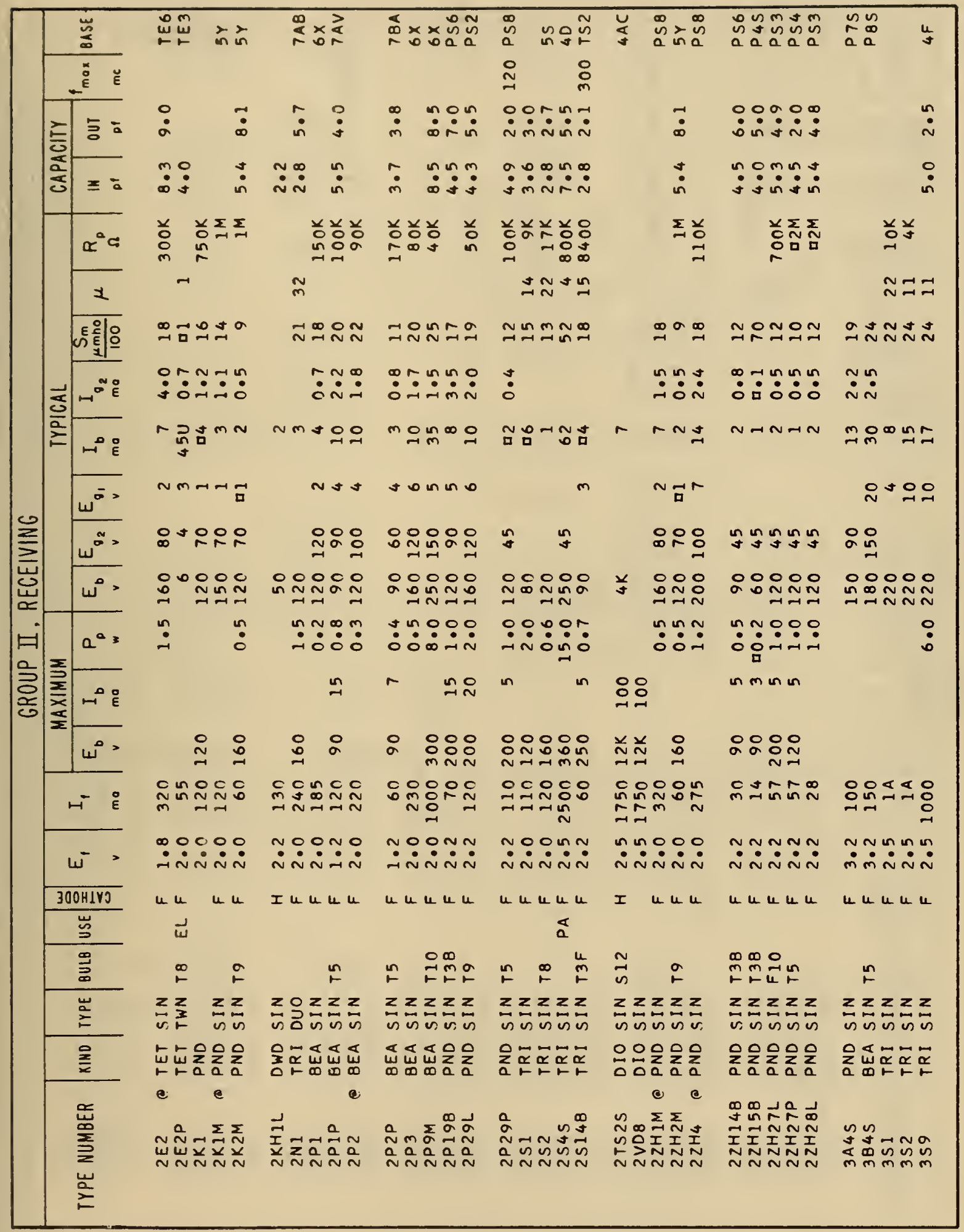




\begin{tabular}{|c|c|c|c|c|c|c|c|c|c|}
\hline & 岕 & 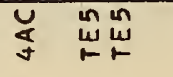 & $\begin{array}{l}\tilde{n} \\
\tilde{a}\end{array}$ & $\stackrel{m}{\leftarrow}$ & ज苟 & $\tilde{a}$ & 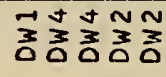 & そุ๊ & E \\
\hline & Eั & & $\stackrel{\circ}{\circ}$ & $\ddot{z}$ & & $\stackrel{\circ}{\sim}$ & & & \\
\hline & 5 & $m: \because \because$ & $\stackrel{ \pm}{a}$ & $\begin{array}{l} \pm: \\
\sim\end{array}$ & & $\because \ddot{j}: \dot{q}$ & & $\stackrel{0}{\infty}$ & $:$ \\
\hline & 兘 & $\stackrel{0}{0}: \stackrel{n}{0}$ & $\stackrel{n}{\infty}$ & $\begin{array}{l}\infty: n \\
\dot{m}=\dot{1}\end{array}$ & & $\begin{array}{l}0: 0 \\
\dot{*}:=\end{array}$ & & $\ddot{i}$ & ? \\
\hline & $x^{a} c$ & 옹 & 产 & 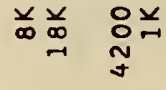 & 무 & $\begin{array}{c}\sum \\
\sim \\
\frac{1}{0} \\
N\end{array}$ & & ․ㅜㅇ & 5 \\
\hline & $\neq$ & 웅요 & : & $\exists \stackrel{\sim}{\sigma} \sigma \cong$ & $\tilde{m}$ & & & & \\
\hline & 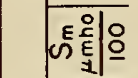 & $\infty \underset{\sim}{\infty}$ & $\stackrel{n}{\sim} \vec{\sim}: 0 \stackrel{\infty}{0}$ & 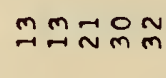 & $\cong$ & $\stackrel{m}{\rightarrow} \underset{N}{ }$ & & in & ก \\
\hline & 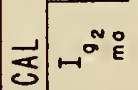 & $\stackrel{n}{\bullet}$ & $\because \because:_{0}$ & & & $\ddot{n} \because \ddot{\sim}$ & 1 & : & : \\
\hline & $\sum_{\bar{\Sigma}}^{\overline{2}}$ & $\neg \ln m_{\square}^{\infty} \infty$ & 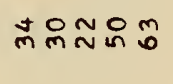 & $\infty+\underset{n}{\infty} \infty \tilde{n}$ & orro & Nrm & 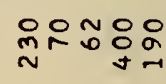 & 응요 & m \\
\hline & $\omega^{\sigma}>$ & 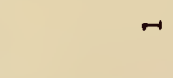 & $\because \exists O^{r}$ & $0+\mathrm{m}$ & $m$ & $\sim r$ & & & \\
\hline$\geqq$ & $\omega^{\approx}>$ & : & 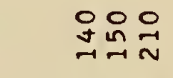 & & & ñ & & ¿ & 8 \\
\hline 岂 & $\omega^{0}=$ & $\begin{array}{r}0 \\
0\end{array}$ & 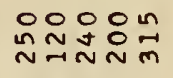 & 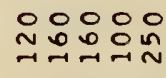 & 원 & 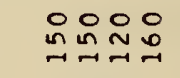 & 品品只足 & 品 & in \\
\hline 曰- & $a^{0}$ & $\ddot{i}:$ & 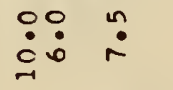 & $\because: 0:$ & $\because \cong$ & $\dot{\sim}:$ & $\begin{array}{l}:: \\
\dot{0} \\
\dot{m}\end{array}$ & $\begin{array}{l}\because: \\
\dot{\sim} \\
\dot{y}\end{array}$ & $\because$ \\
\hline \begin{tabular}{|c|}
$\overline{3}$ \\
온
\end{tabular} & 产 & 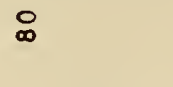 & in & & i : & $\underset{\overbrace{}}{\stackrel{N}{N}=}$ & 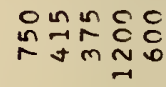 & 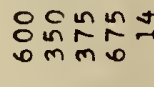 & \\
\hline & $w^{0}>$ & 恙 & $\stackrel{\circ}{\sim}$ & : & 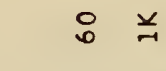 & 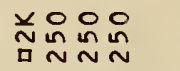 & 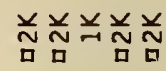 & 并并寺忈品 & \\
\hline & Н & 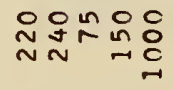 & 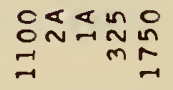 & 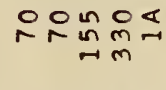 & 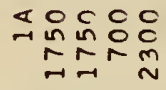 & : $\underset{\sim}{\sim} \underset{\sim}{N}:$ & 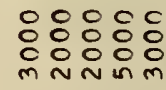 & 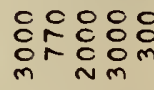 & \\
\hline & $\omega^{-}>$ & 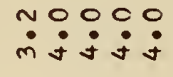 & 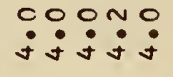 & 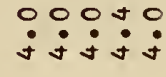 & 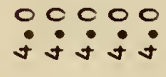 & $\because \because \because: 0$ & $\begin{array}{l}0: 0: C \\
\dot{n} \operatorname{in} \text { in in }\end{array}$ & 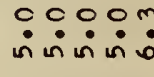 & \\
\hline & 30OHIYJ & IIиนI & Iu & uUuIu & $I u и u u$ & IIII & แIIII & I I & I \\
\hline & 岁 & & $\overleftarrow{a}$ & & & $\frac{u}{\alpha}$ & & 3 & 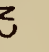 \\
\hline & $\stackrel{9}{\Xi}$ & $\stackrel{t}{\leftarrow}$ & $\stackrel{ }{\circ}$ & & 욤다 & 운운 & 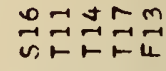 & $\stackrel{m}{2}$ & $n$ \\
\hline & $\stackrel{4}{2}$ & 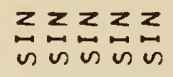 & 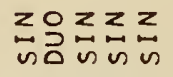 & $\begin{array}{l}z \geq z z z \\
\text { 致的的 }\end{array}$ & $\begin{array}{l}z \geq z \geq z \\
\text { vivus }\end{array}$ & $\begin{array}{l}z \geq z \geq z \\
\text { 的的的 }\end{array}$ & 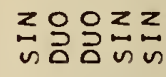 & 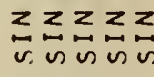 & \\
\hline & 옾 & 음음ㄴّ & 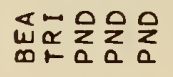 & 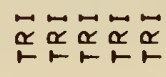 & 똔음움음음 & 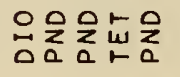 & 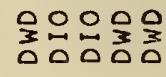 & 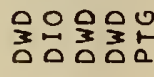 & \\
\hline & 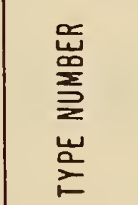 & 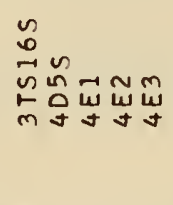 & 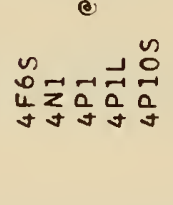 & 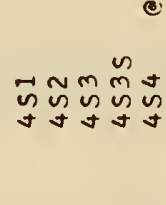 & 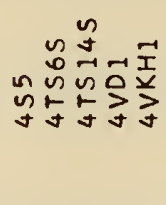 & 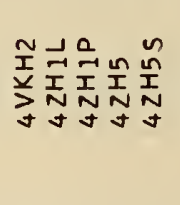 & 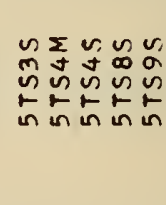 & 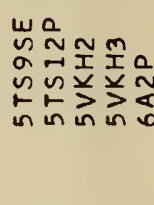 & \\
\hline
\end{tabular}




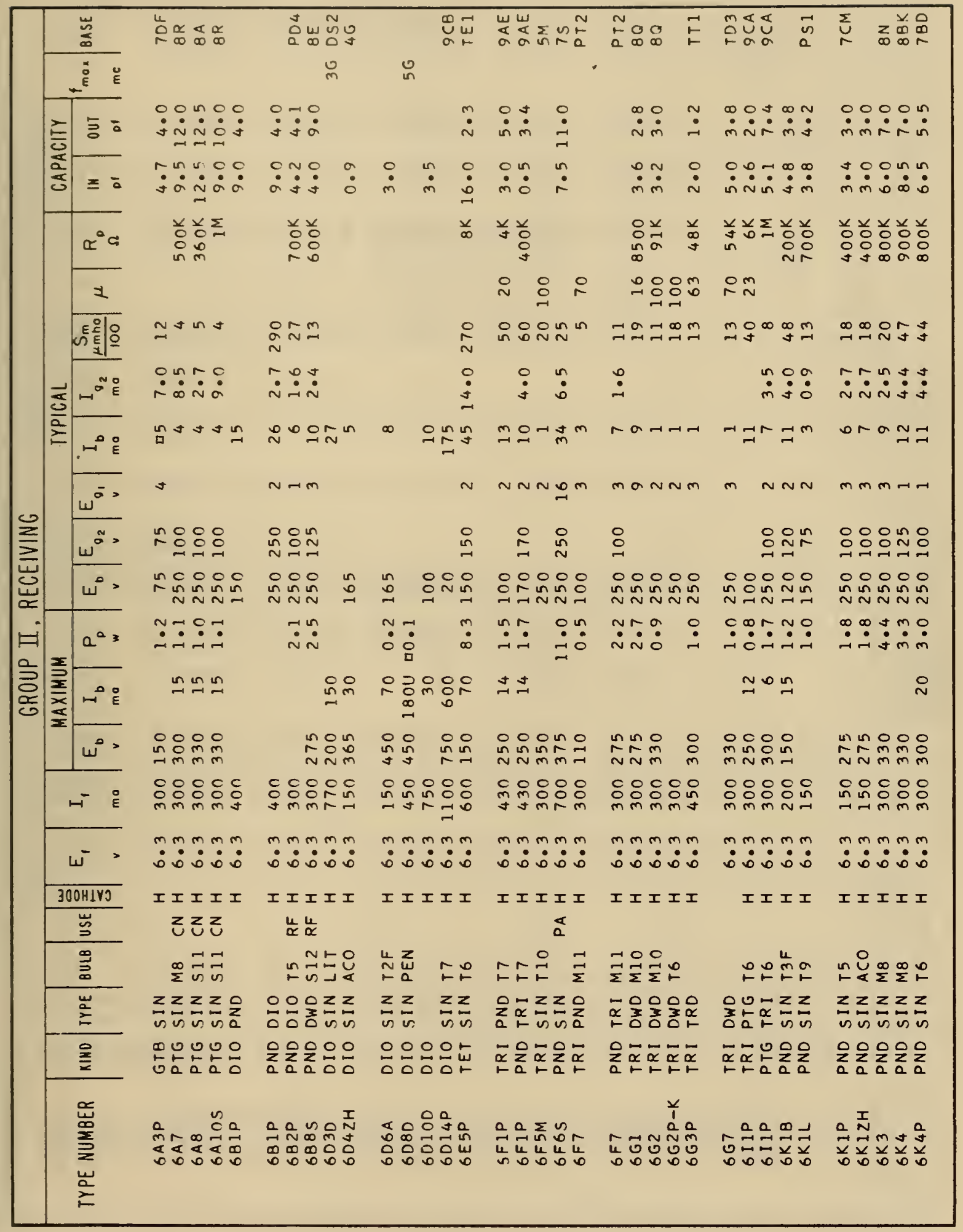




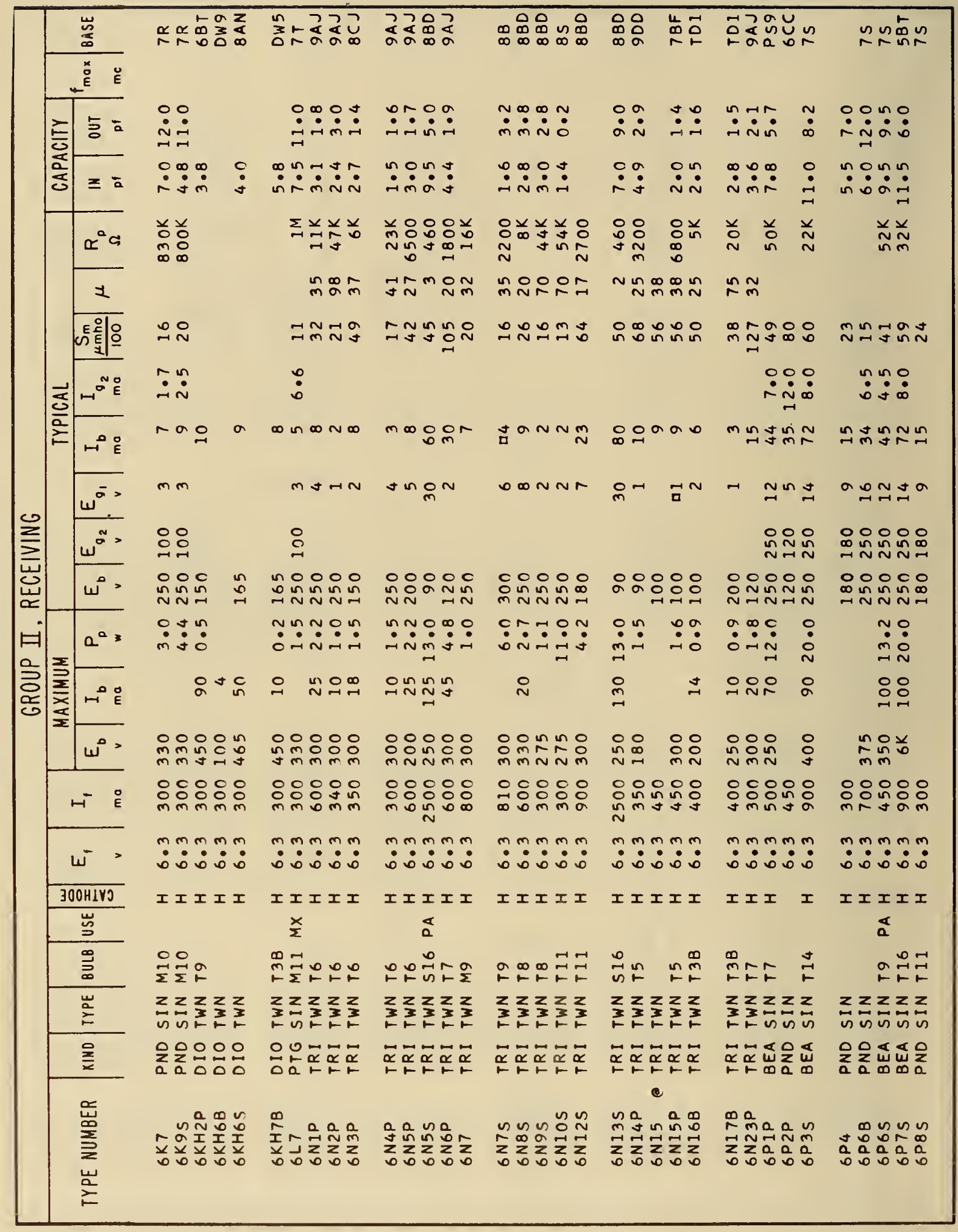









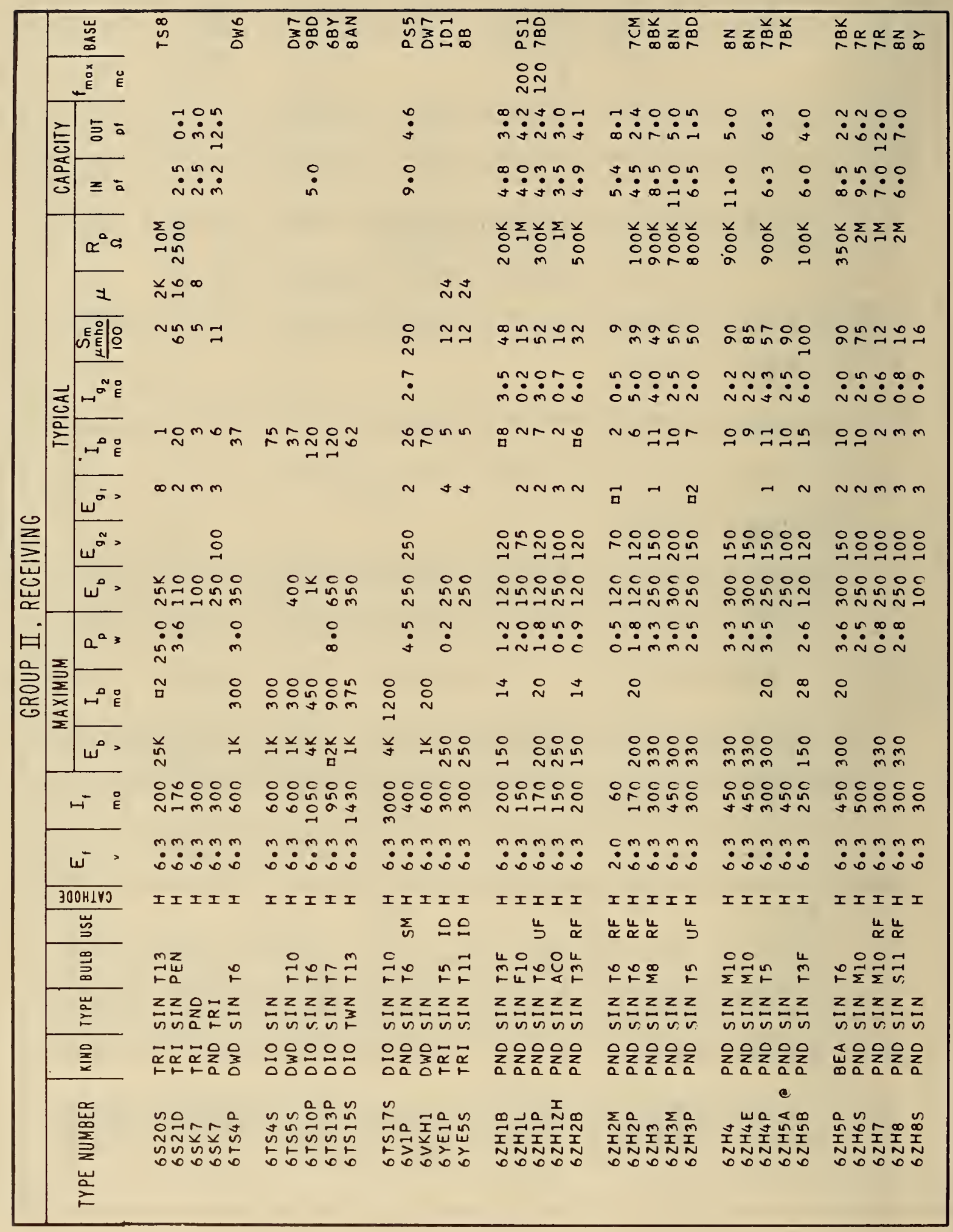




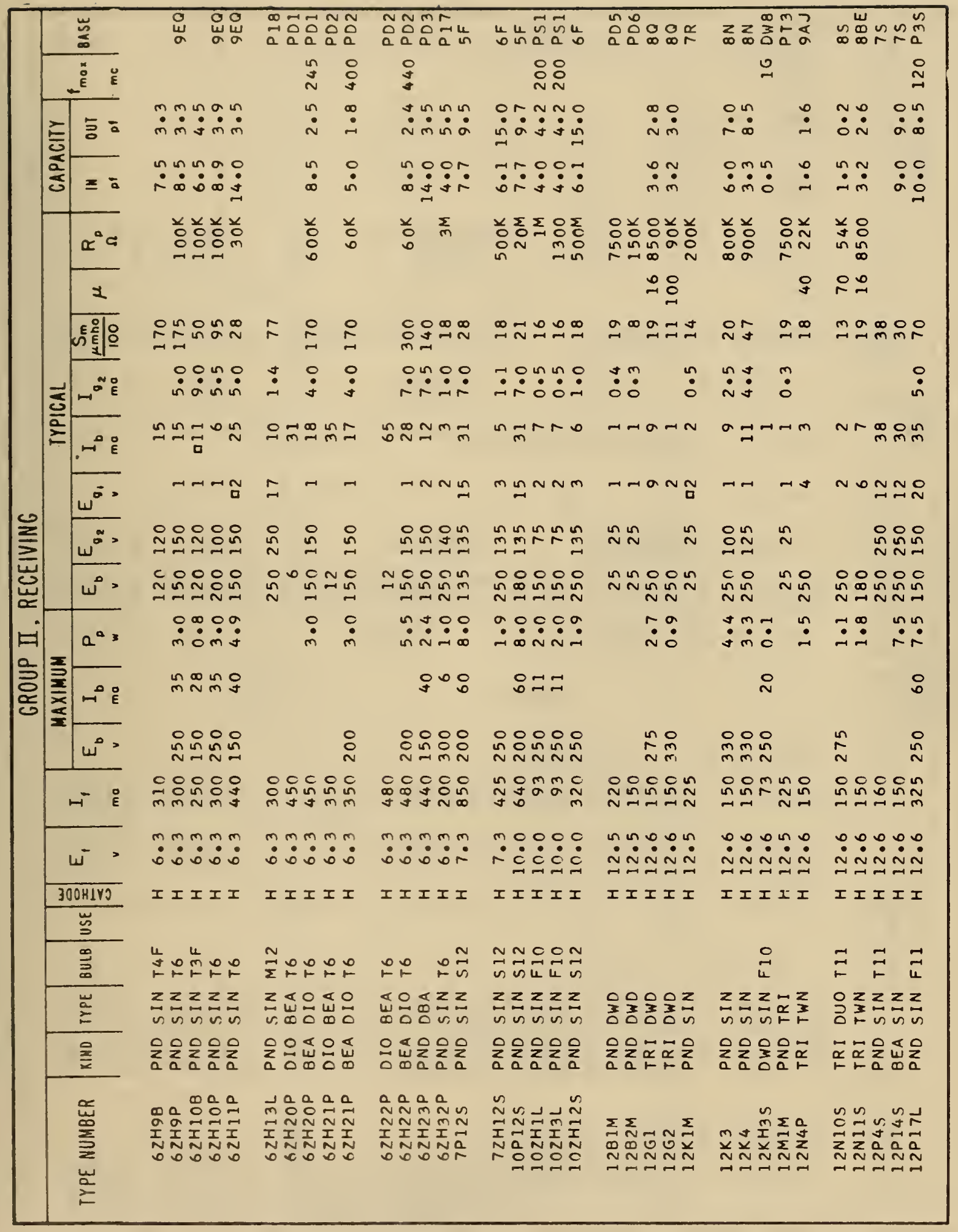




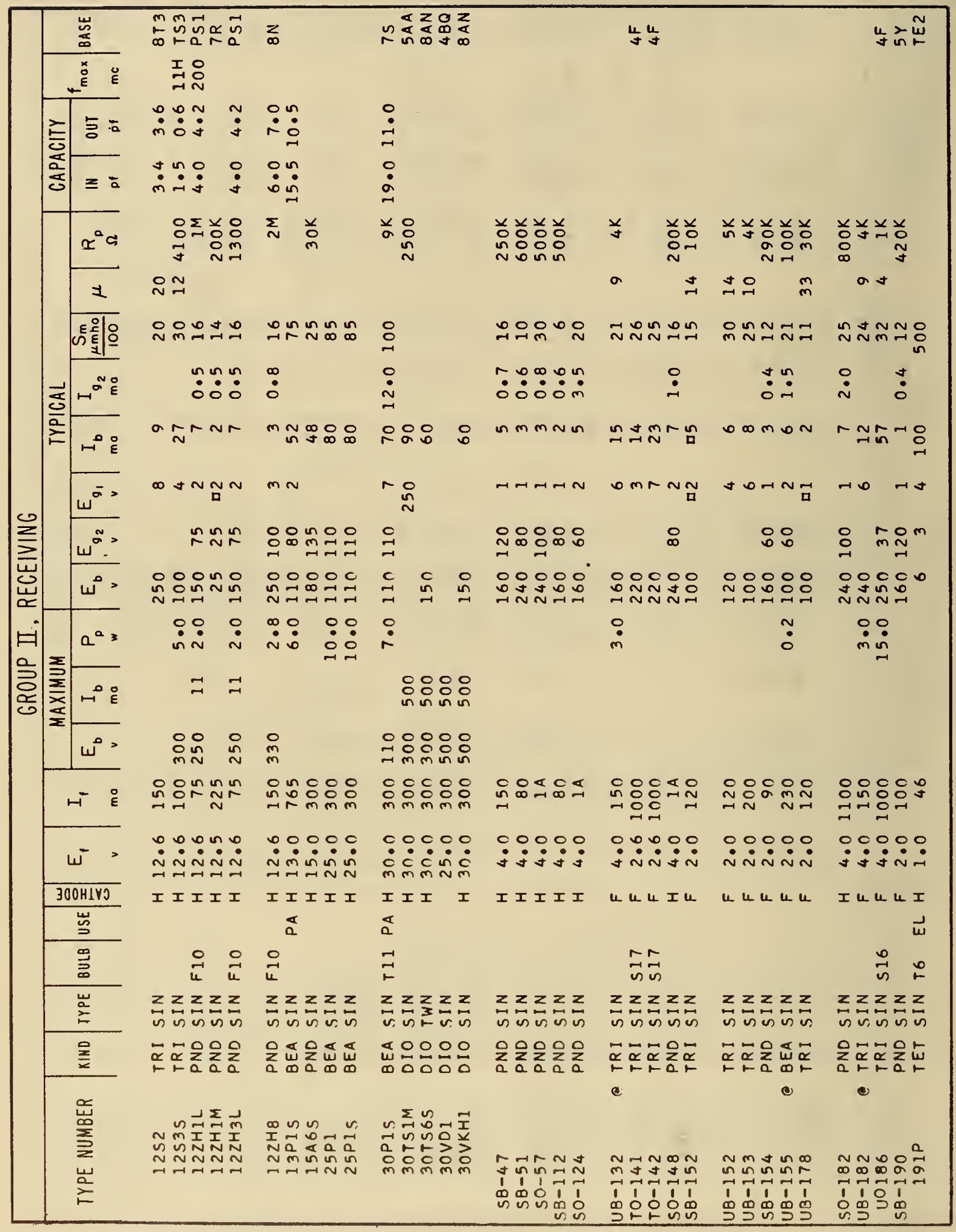




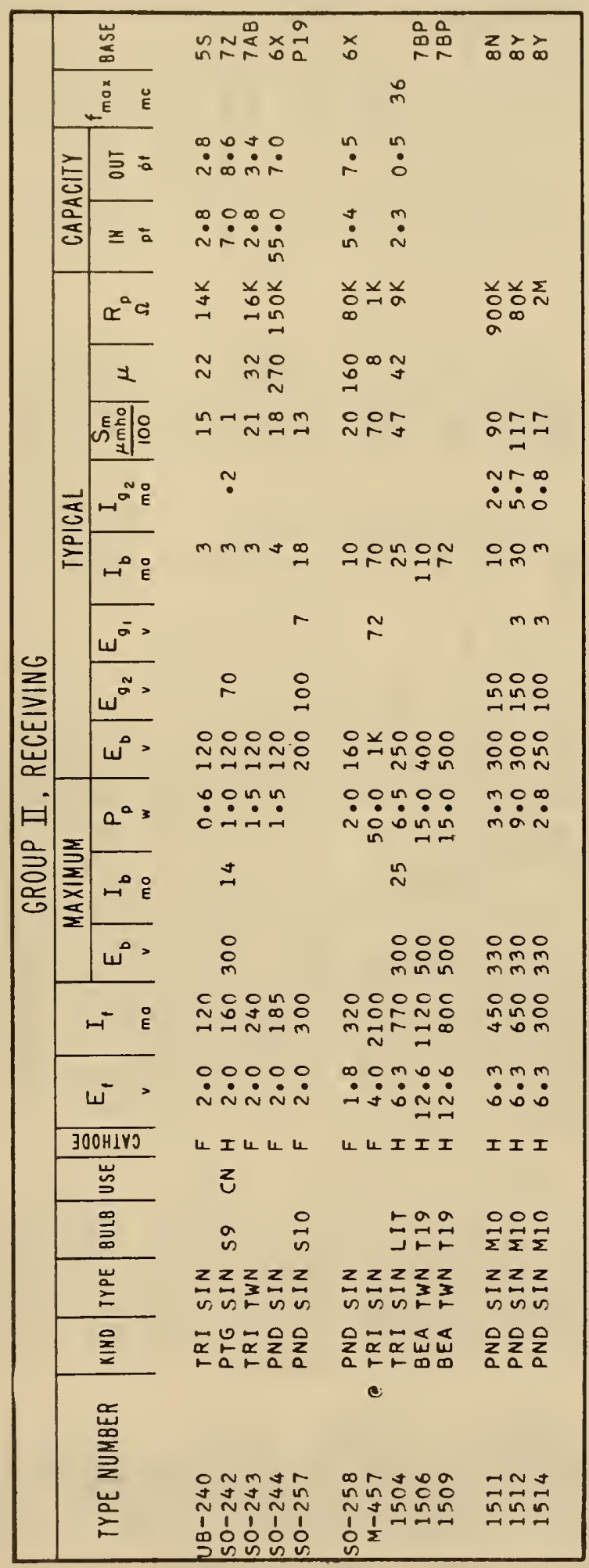




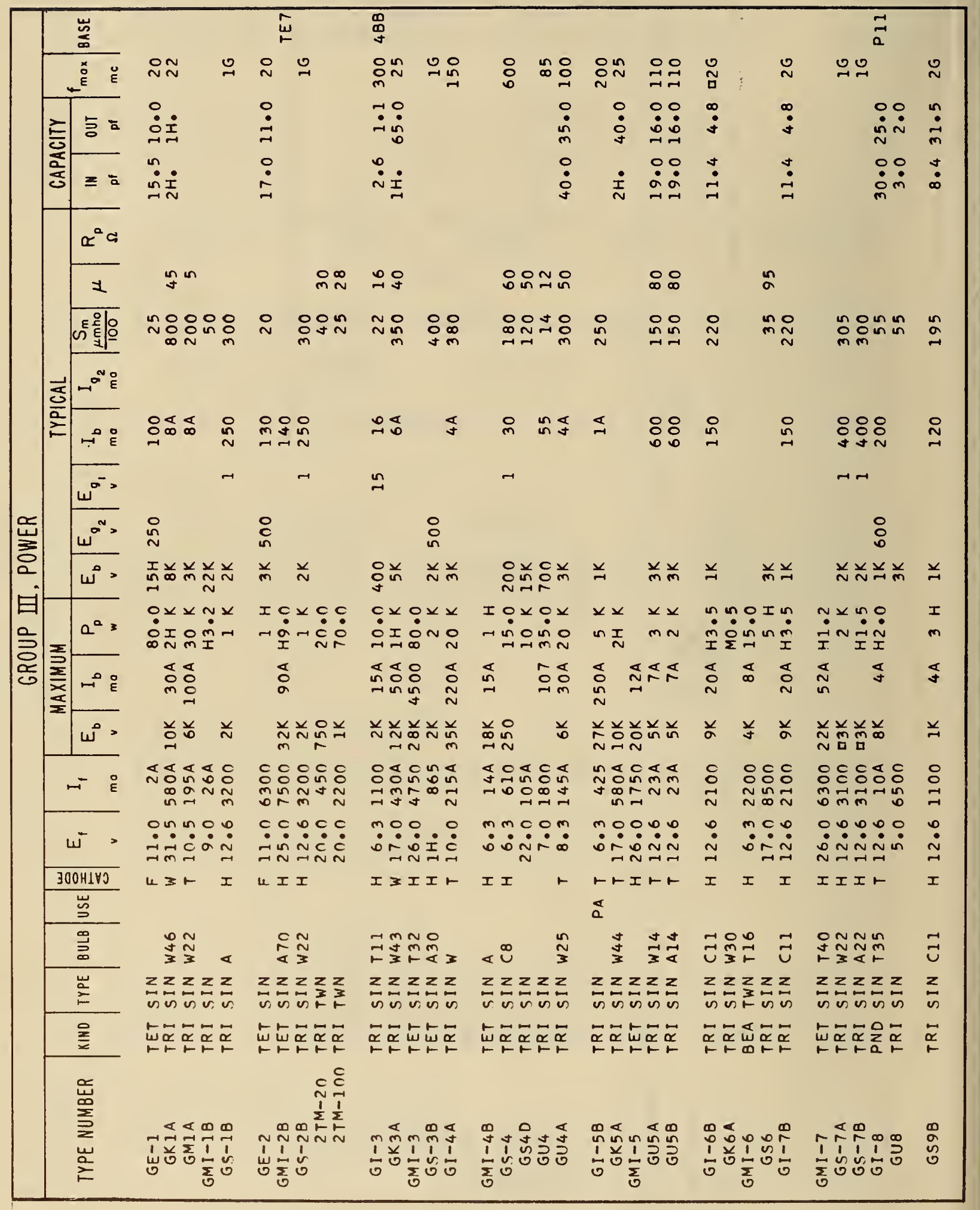




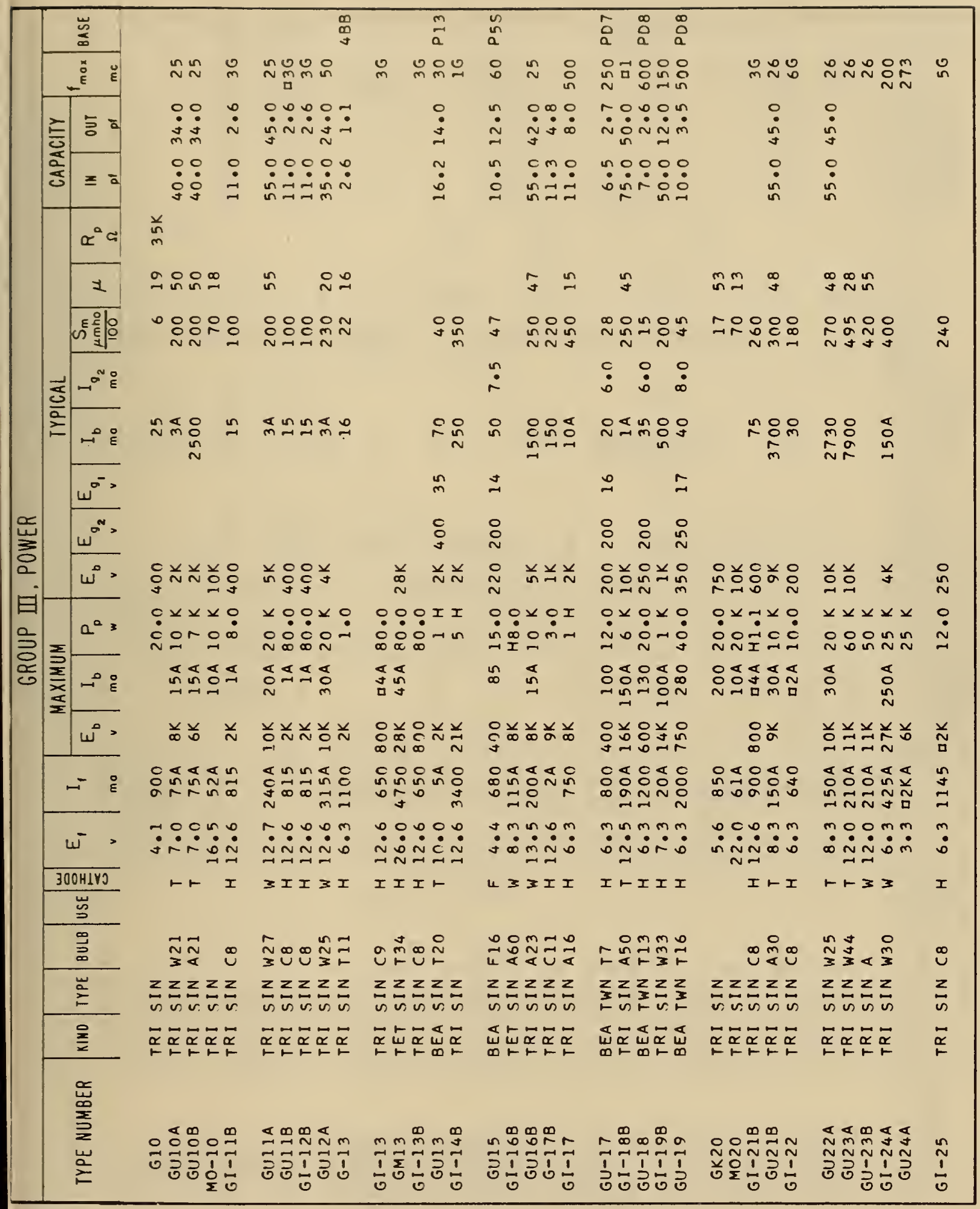




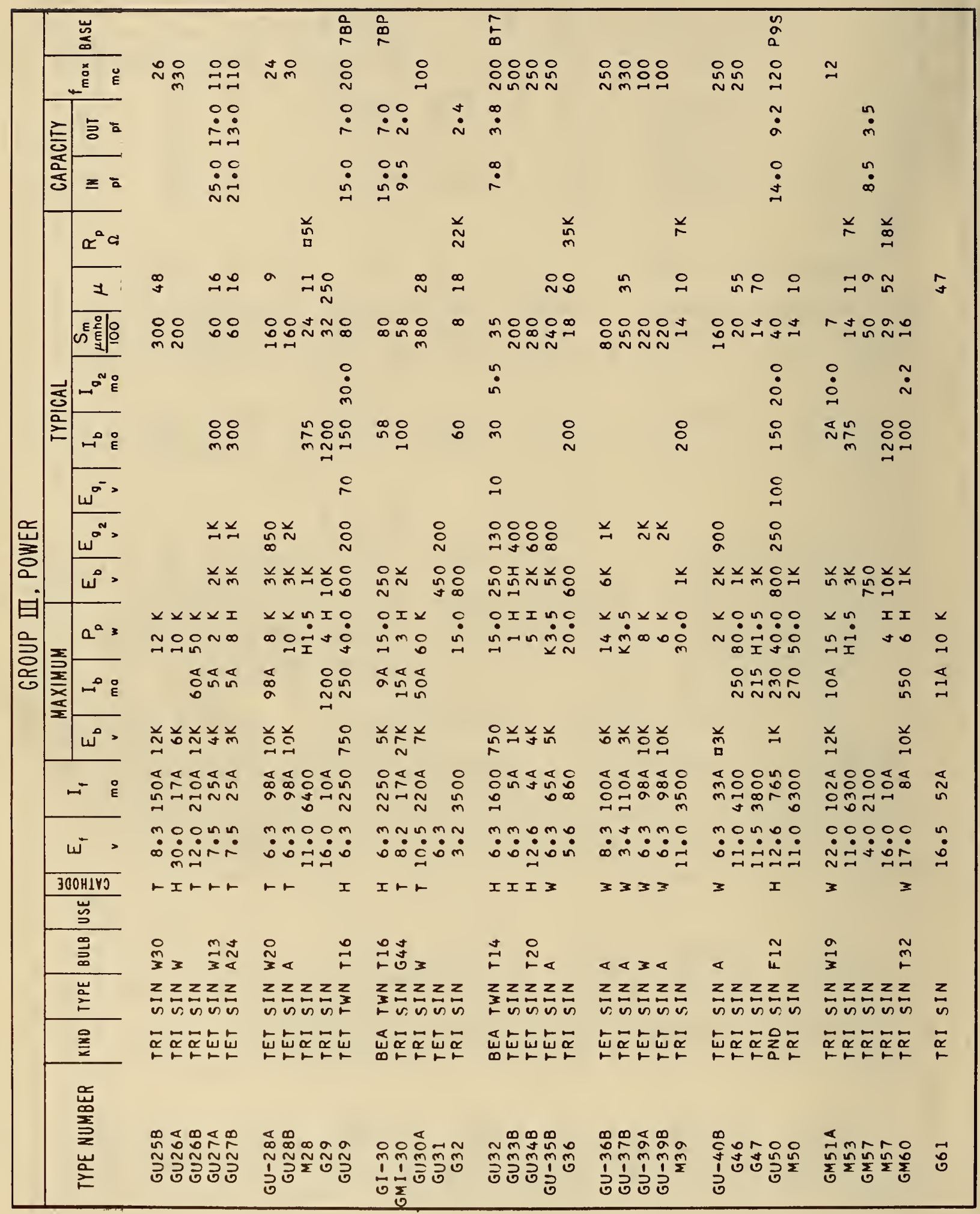




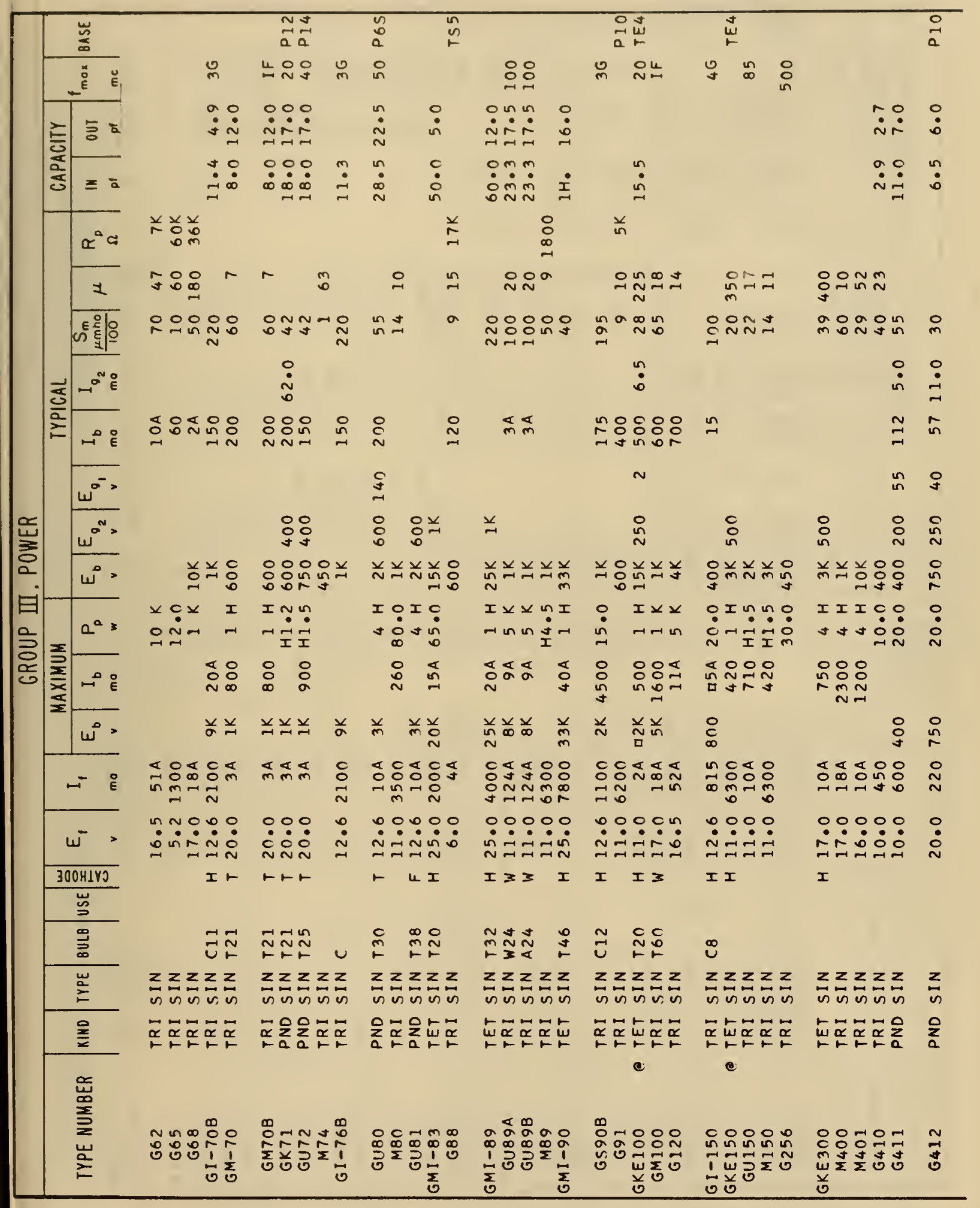




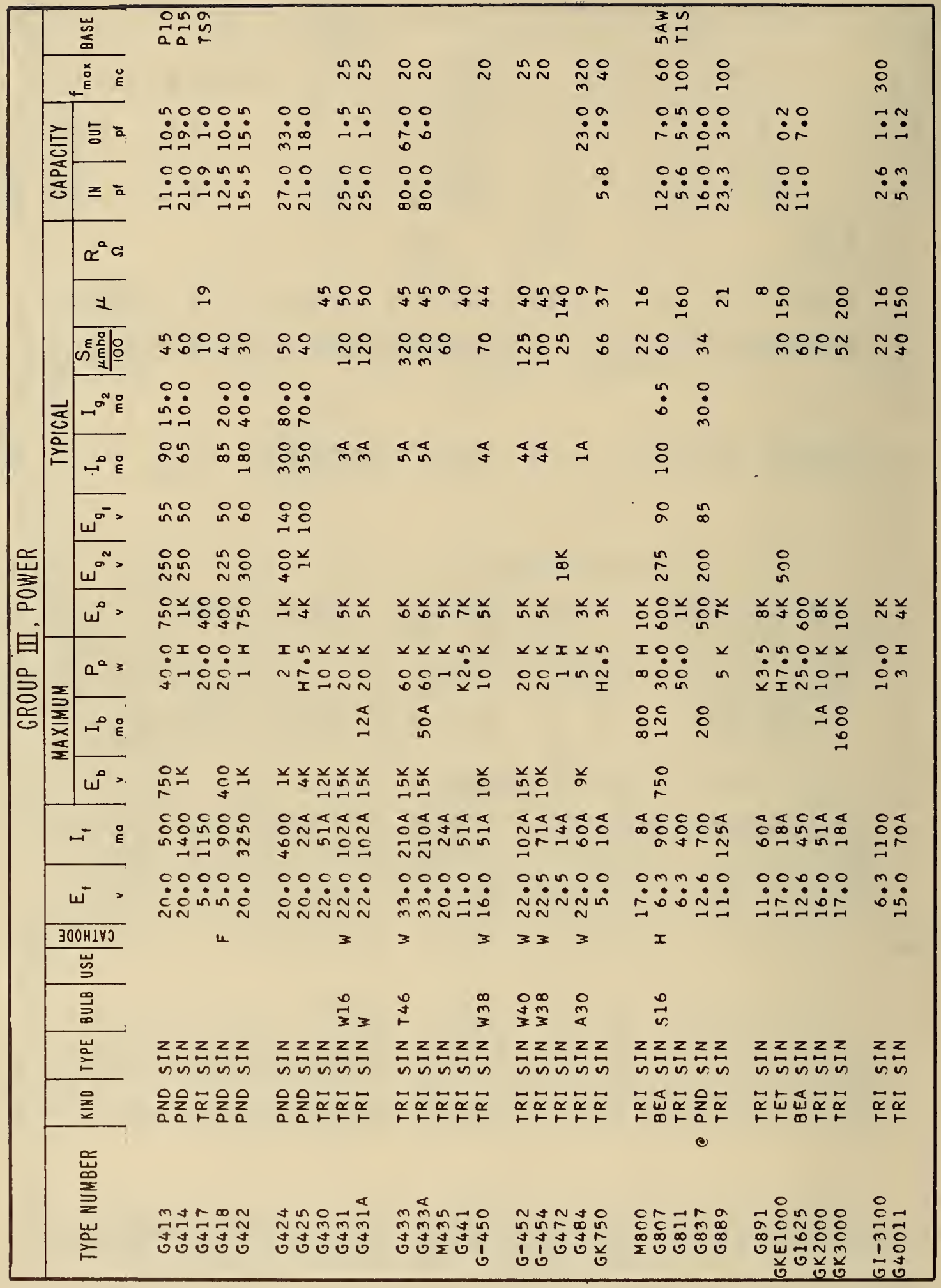




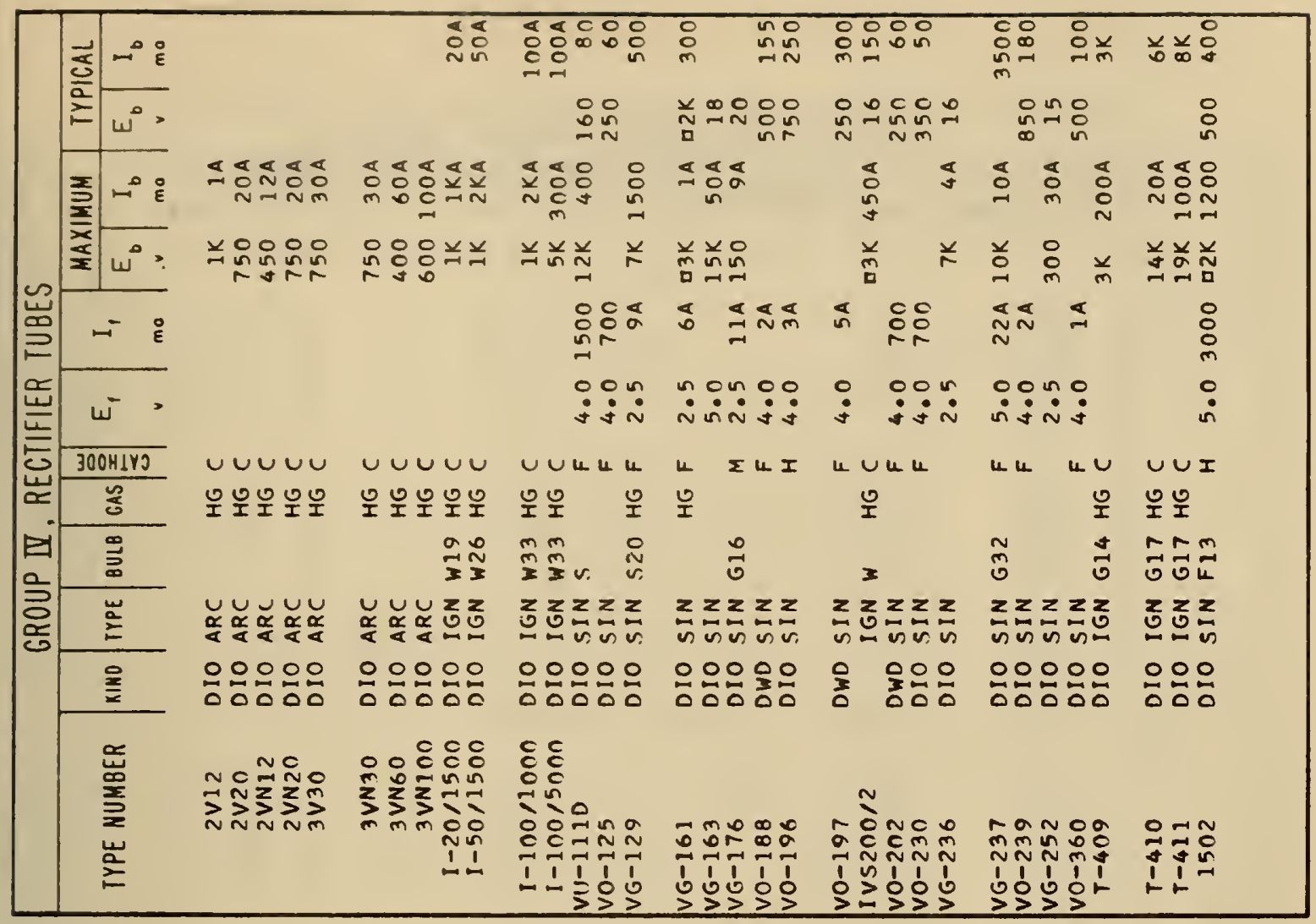

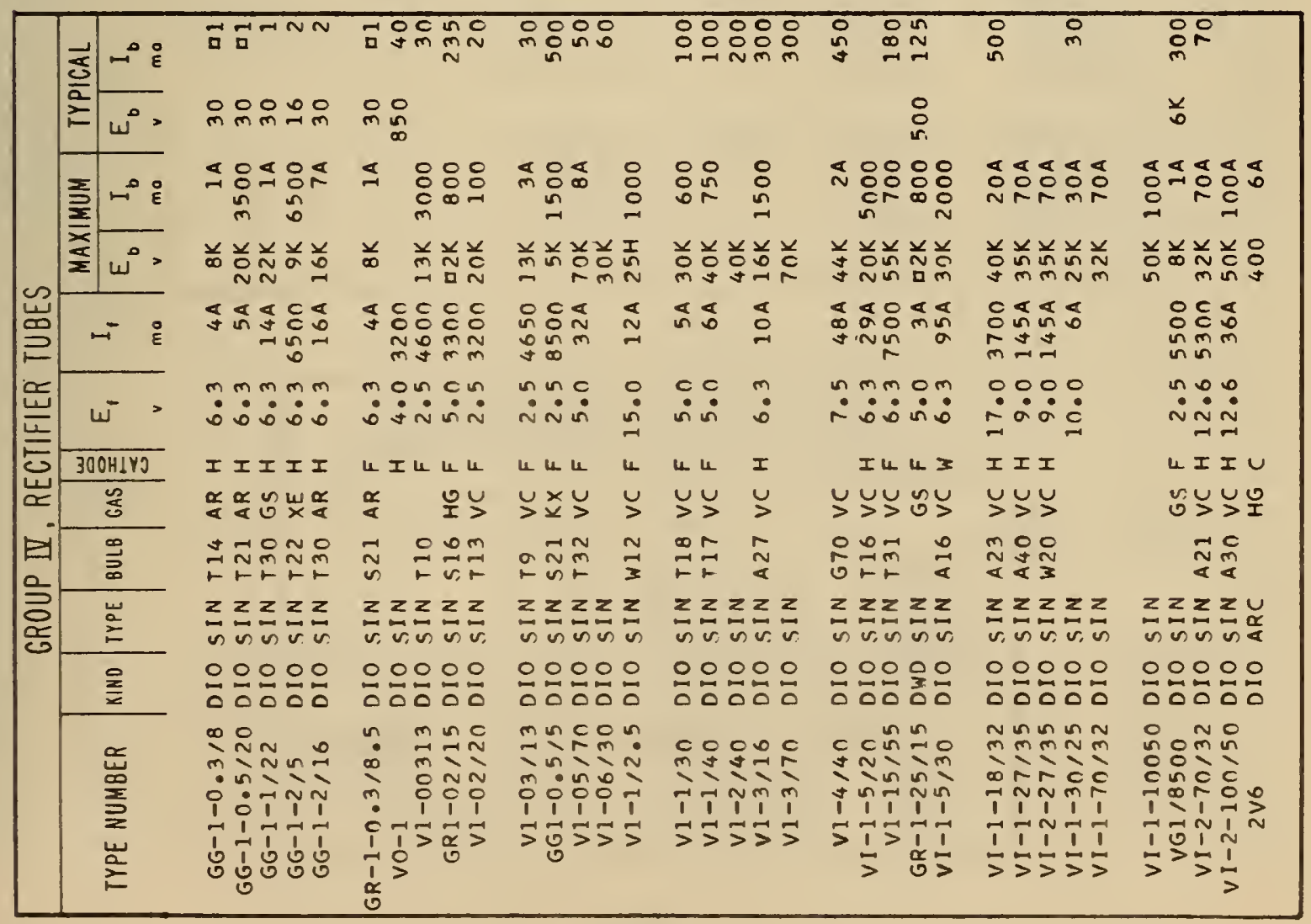




\begin{tabular}{|c|c|c|c|c|c|c|c|c|c|c|c|}
\hline \multirow[b]{3}{*}{ TYPE NUABER } & \multirow{3}{*}{ GROL } & \multirow{2}{*}{\multicolumn{2}{|c|}{$\frac{I P}{G A S}$}} & \multirow{3}{*}{$\begin{array}{l}\text { CATH } \\
\text { MATL }\end{array}$} & \multirow{2}{*}{\multicolumn{2}{|c|}{ VOLT.RANGE }} & \multirow{2}{*}{\multicolumn{2}{|c|}{ CUR. RANGE }} & \multirow{2}{*}{\multicolumn{2}{|c|}{ DIMEN }} & \multirow[b]{3}{*}{ BASE } \\
\hline & & & & & & & & & & & \\
\hline & & KIND & $\begin{array}{l}\text { PRES } \\
\mathrm{mm}\end{array}$ & & $\begin{array}{c}\max \\
v\end{array}$ & $\begin{array}{c}n \mid N \\
v\end{array}$ & $\begin{array}{l}\text { max } \\
\text { mo }\end{array}$ & $\begin{array}{l}\text { MIN } \\
\text { mo }\end{array}$ & $\begin{array}{l}01 \mathrm{~A} . \\
\mathrm{mm}\end{array}$ & $\begin{array}{l}\text { LTH } \\
\mathrm{mm}\end{array}$ & \\
\hline SGIP & REG & AHE & & & 190 & 145 & 30 & 5 & 22 & 65 & 7DN \\
\hline$S G 1 P-V$ & REG & & & & 170 & 145 & 30 & 5 & 22 & 65 & $7 D N$ \\
\hline SGIP-YE & REG & & & & 170 & 143 & 30 & 5 & 22 & 65 & $7 D N$ \\
\hline SG2P & REG & $A K N$ & & & 150 & 104 & 30 & 5 & 22 & 65 & $7 D N$ \\
\hline SG2S & REG & NA & 30 & & 110 & 70 & 40 & 5 & 32 & 75 & $4 A J$ \\
\hline SG $3 P$ & REG & AHE & & & 170 & 144 & 40 & 5 & 22 & 65 & \\
\hline SG3S & REG & $A H N$ & 30 & & 133 & 105 & 40 & 5 & 32 & 75 & $4 \mathrm{AJ}$ \\
\hline SG4S & REG & AHE & 30 & N1 & 180 & 145 & 30 & 5 & 32 & 75 & $4 \mathrm{AJ}$ \\
\hline SG5B & REG & AHE & & & 190 & 142 & 10 & 5 & 10 & 36 & \\
\hline SG5B-V & REG & & & & 170 & 142 & 10 & 5 & 10 & 36 & \\
\hline SG7S & REG & & & & 480 & 390 & 0.1 & 0.003 & & & \\
\hline SG8S & REG & & & & 970 & 880 & 0.1 & 0.003 & 10 & & \\
\hline SG9S & REG & & & & $13 \mathrm{H}$ & $12 \mathrm{H}$ & 0.1 & 0.01 & 10 & & \\
\hline SGIOS & REG & NK & & & 150 & 86 & 15 & 4 & 32 & 40 & \\
\hline SG $13 P$ & REG & $A H N$ & 55 & NI & 180 & 143 & 30 & 5 & 19 & 55 & 7DN \\
\hline SG15P & REG & $A H N$ & 54 & MO & 150 & 104 & 30 & 5 & 19 & 55 & $70 N$ \\
\hline SG $16 P$ & REG & NA & 40 & MO & 130 & 80 & 30 & 5 & 19 & 55 & $70 N$ \\
\hline SG17S & REG & NEH & & & 1350 & 850 & 60 & 10 & 38 & 189 & \\
\hline SGIBS & REG & NEH & & & 1500 & 950 & 60 & 10 & 38 & 189 & \\
\hline SG19S & REG & $\mathrm{NEH}$ & & & 1650 & 1050 & 60 & 10 & 38 & 189 & \\
\hline SG201S & REG & NK & 50 & MO & 150 & 86 & 15 & 4 & 32. & 40 & $70 N$ \\
\hline SG202B & REG & NA & 35 & MO & 140 & 81 & 5 & 1.5 & 10 & 40 & \\
\hline SG226 & REG & NE & & & 95 & 70 & 40 & 8 & 50 & 130 & \\
\hline SG227 & $R E G$ & NE & & & 95 & 70 & 60 & 10 & 65 & 135 & \\
\hline SG301S & REG & HY & 16 & NI & 439 & 380 & 0.1 & 0.003 & 12 & 55 & \\
\hline SG302S & REG & HY & 32 & NI & 970 & 880 & 0.1 & 0.003 & 12 & 55 & \\
\hline SG303S & REG & HY & 143 & N 1 & 1350 & 1220 & 0.1 & 0.01 & 12 & 55 & \\
\hline SG304S & REG & HY & & & 4200 & 3800 & 1 & 0.05 & 25 & 129 & \\
\hline SG305K & REG & & & & $10 K$ & $9 k$ & 1.5 & 0.05 & 33 & 180 & \\
\hline SG $306 \mathrm{~K}$ & REG & & & & $26 K$ & $24 K$ & 1.5 & 0.05 & 48 & 245 & \\
\hline
\end{tabular}

\begin{tabular}{|c|c|c|c|c|c|c|c|c|}
\hline \multicolumn{9}{|c|}{ GROUP VI, CURRENT REGULATOR TUBES } \\
\hline \multirow[b]{2}{*}{ TYPE NUMBER } & \multirow[b]{2}{*}{ KINO } & \multirow[b]{2}{*}{ TYPE } & \multirow[b]{2}{*}{ BULB } & \multicolumn{2}{|c|}{ VOLT.RANGE } & \multicolumn{2}{|c|}{ CUR. RANGE } & \multirow[b]{2}{*}{ BASE } \\
\hline & & & & $\begin{array}{c}\text { MAX } \\
v\end{array}$ & $\underset{v}{M I N}$ & $\begin{array}{c}\text { MAX } \\
\text { mo }\end{array}$ & $\begin{array}{l}\text { MIN } \\
\text { mo }\end{array}$ & \\
\hline $24 \mathrm{~B} 12-18$ & BAL & $S I N$ & T10 & 18 & 12 & & 255 & \\
\hline $3 B 17-35$ & $B A L$ & SIN & 714 & 35 & 17 & & 300 & $8 E S$ \\
\hline $\begin{array}{l}3865-135 \\
425 B 55-12\end{array}$ & $\begin{array}{l}\text { BAL } \\
\text { BAL }\end{array}$ & $\begin{array}{l}\operatorname{SIN} \\
\operatorname{SIN}\end{array}$ & $T 14$ & $\begin{array}{r}135 \\
12\end{array}$ & $\begin{array}{l}65 \\
\square 6\end{array}$ & 460 & $\begin{array}{l}300 \\
425\end{array}$ & $\begin{array}{l}8 E S \\
8 E S\end{array}$ \\
\hline $85855-12$ & $B A L$ & $\operatorname{SIN}$ & T9 & 12 & $\square 6$ & 920 & 780 & $8 E S$ \\
\hline $\begin{array}{l}185-9 \\
1810-17\end{array}$ & $\begin{array}{l}B A L \\
B A L\end{array}$ & $\begin{array}{l}\operatorname{SIN} \\
\operatorname{SIN}\end{array}$ & $\begin{array}{l}T 14 \\
T 14\end{array}$ & $\begin{array}{r}9 \\
17\end{array}$ & $\begin{array}{r}5 \\
10\end{array}$ & & $\begin{array}{l}1000 \\
1000\end{array}$ & $\begin{array}{l}\text { DS } 5 \\
\text { DS } 5\end{array}$ \\
\hline ST $2 S$ & $B A L$ & TWN & & 17 & 6 & & 2000 & DS6 \\
\hline ST $3 P$ & $B A L$ & SIN & T6 & 6 & 4 & 880 & 720 & \\
\hline
\end{tabular}




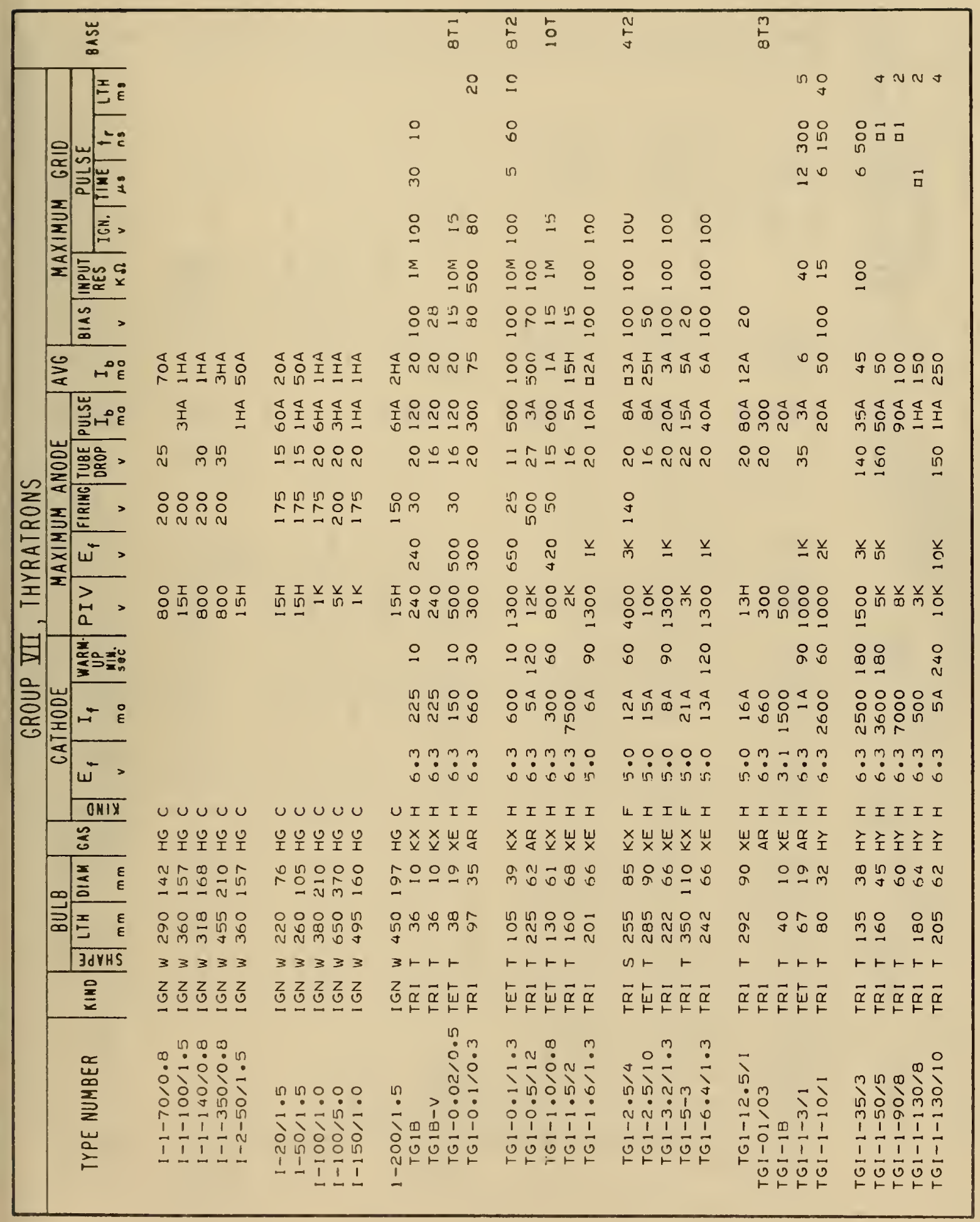




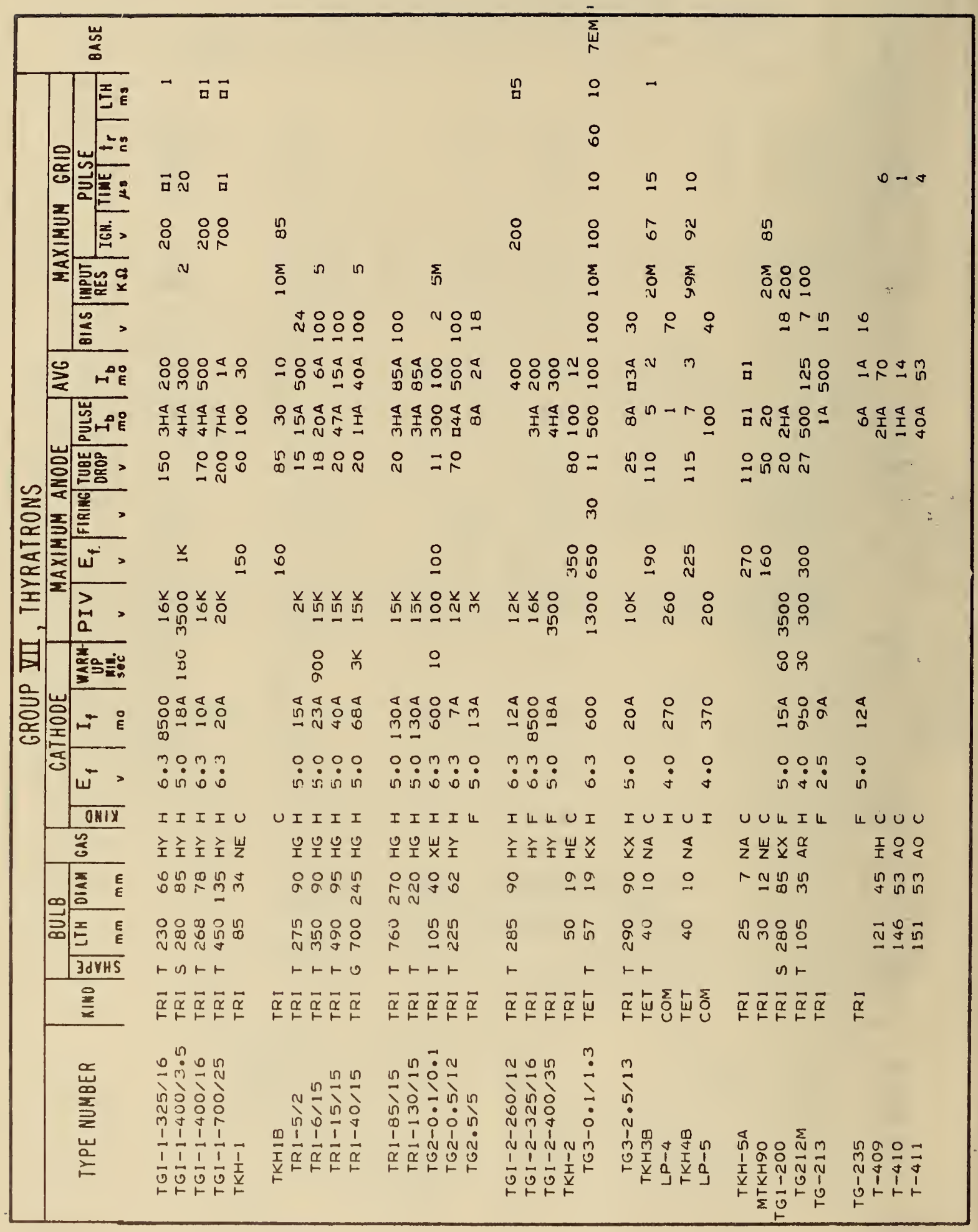




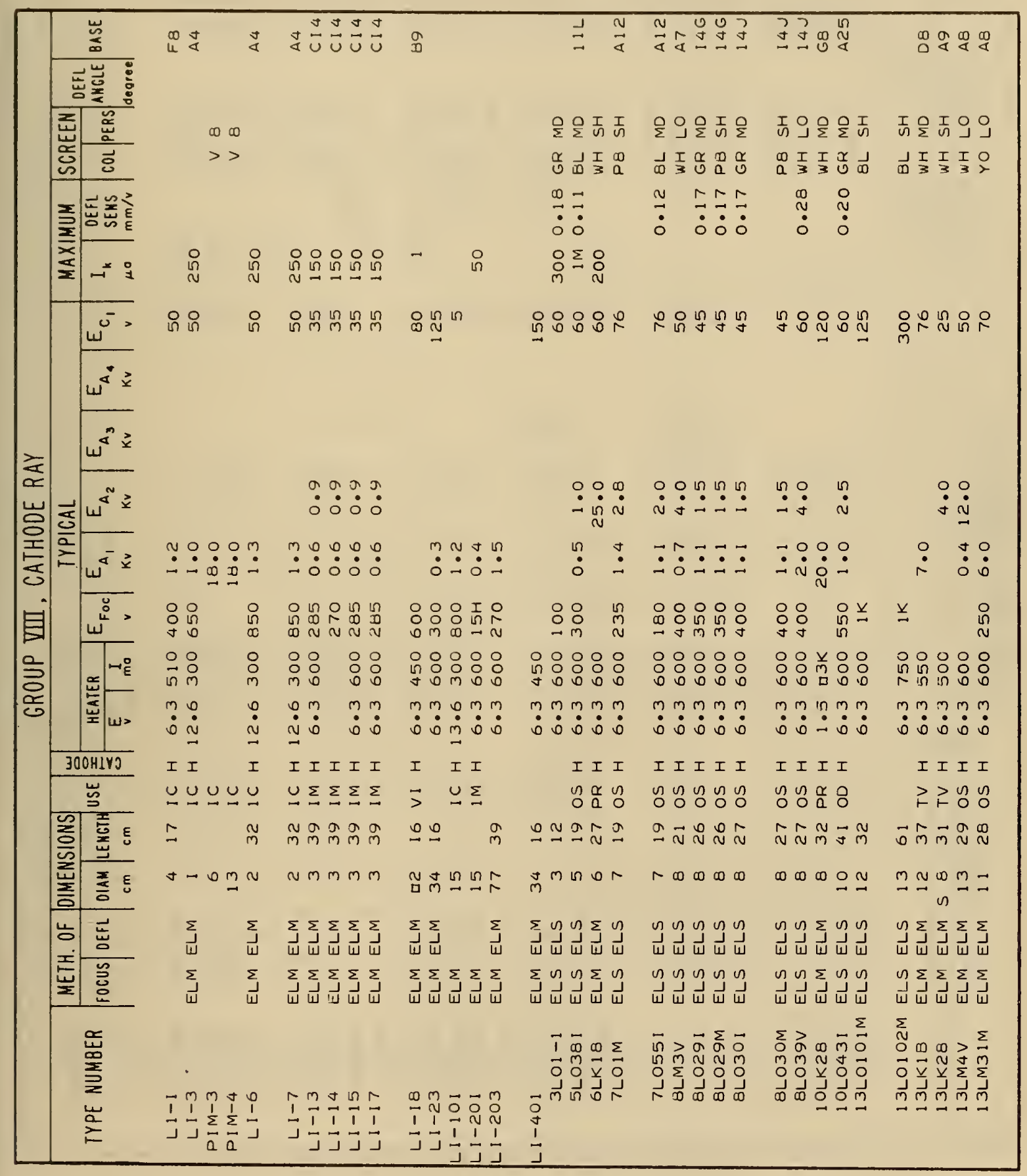




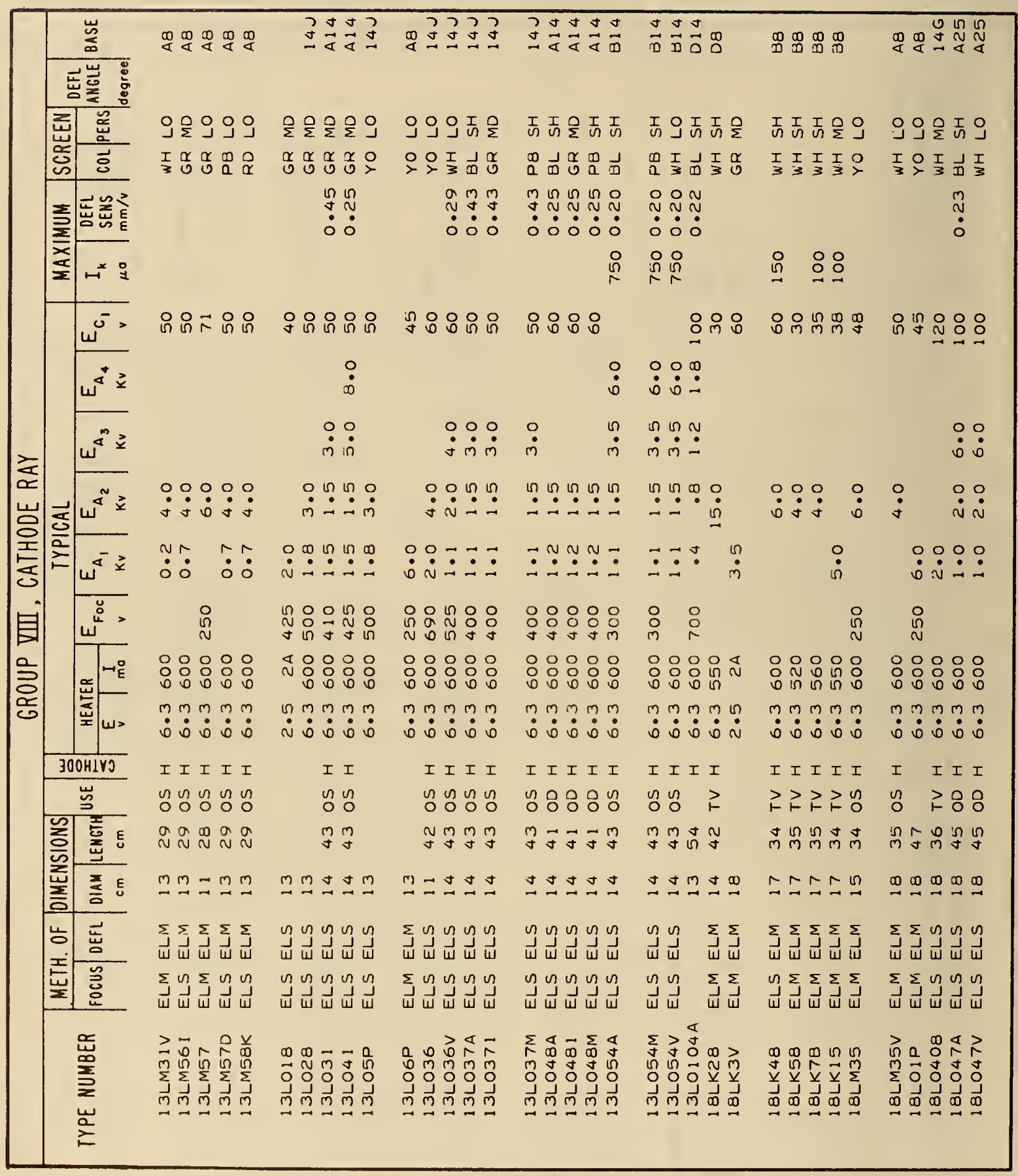




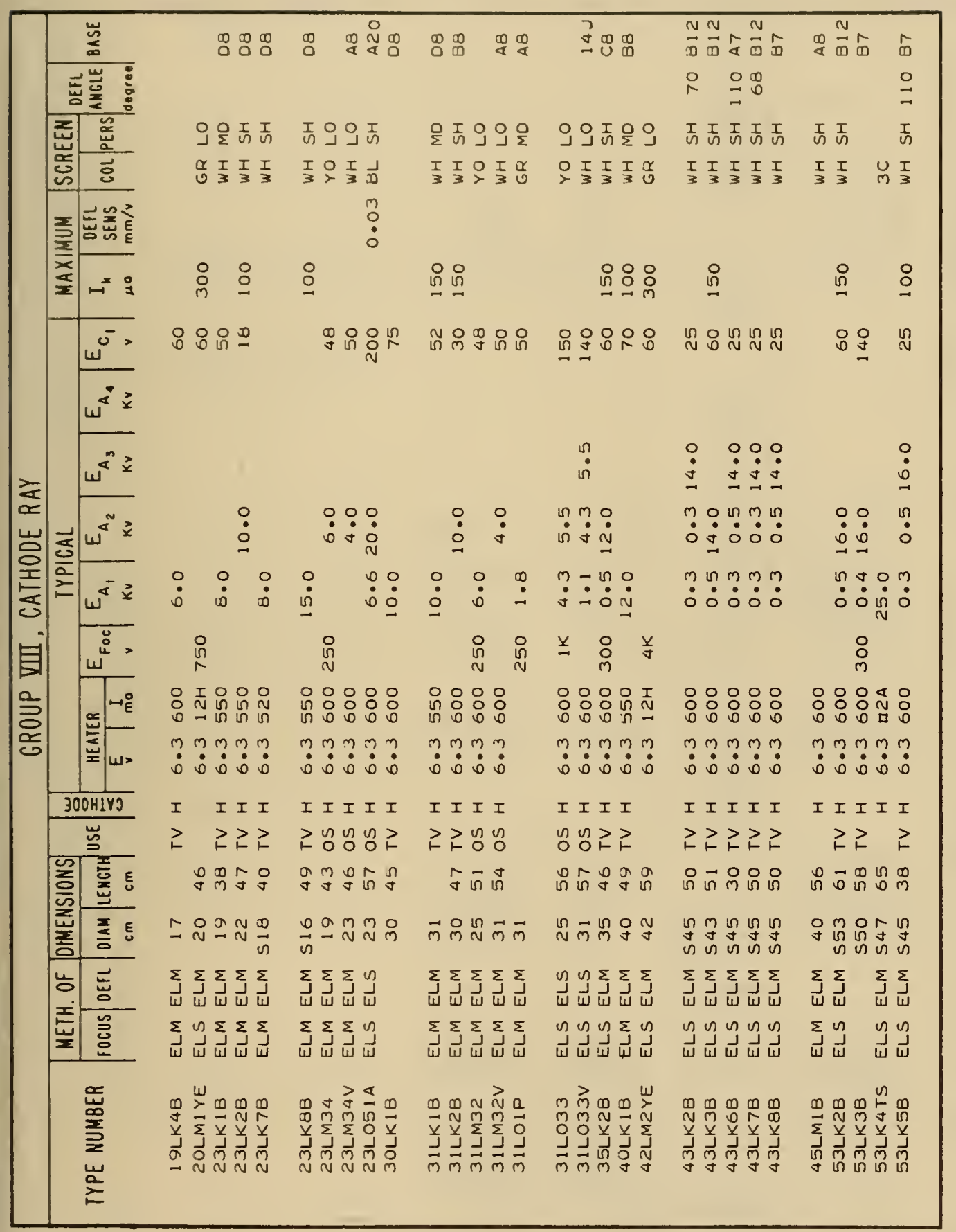




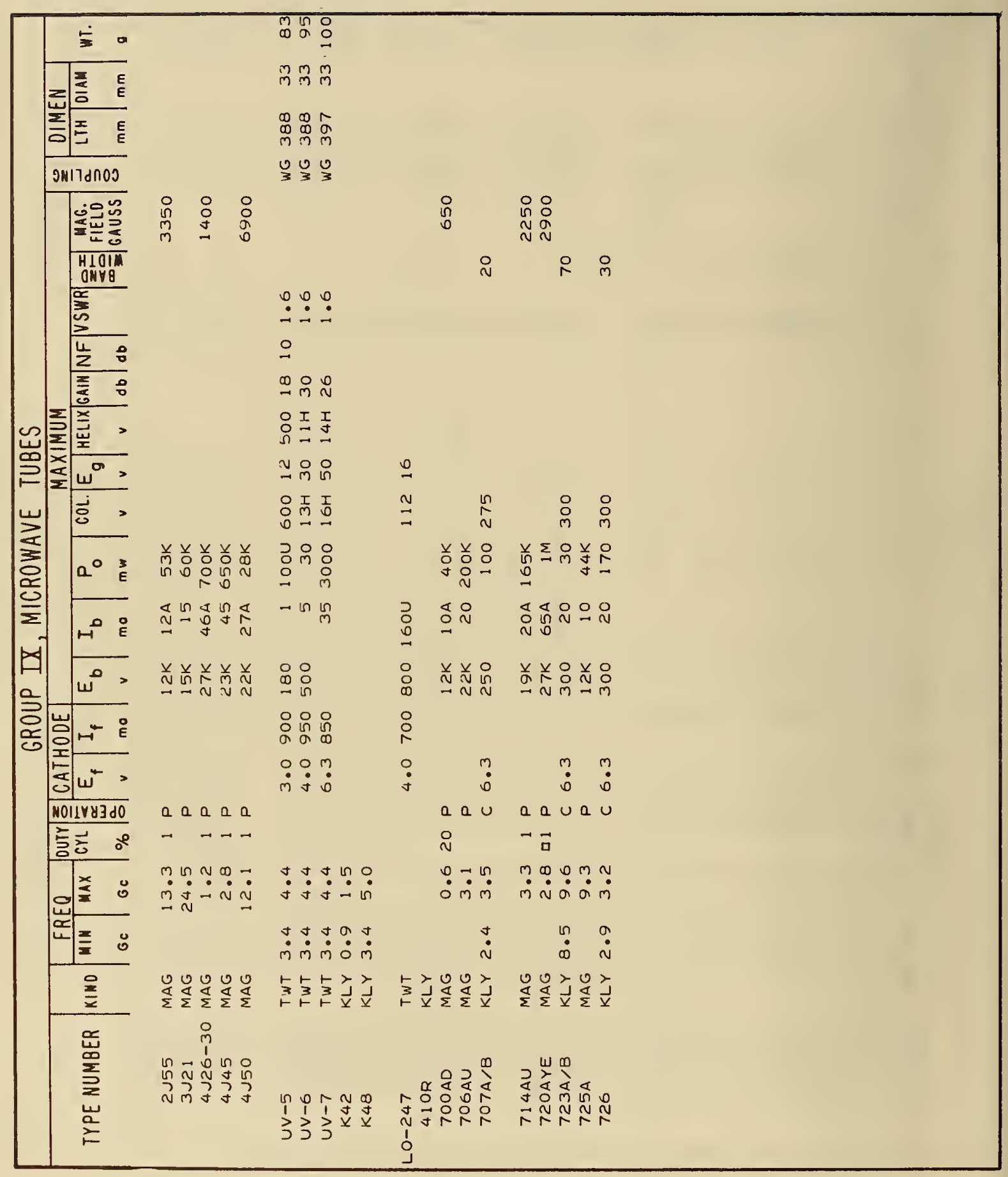




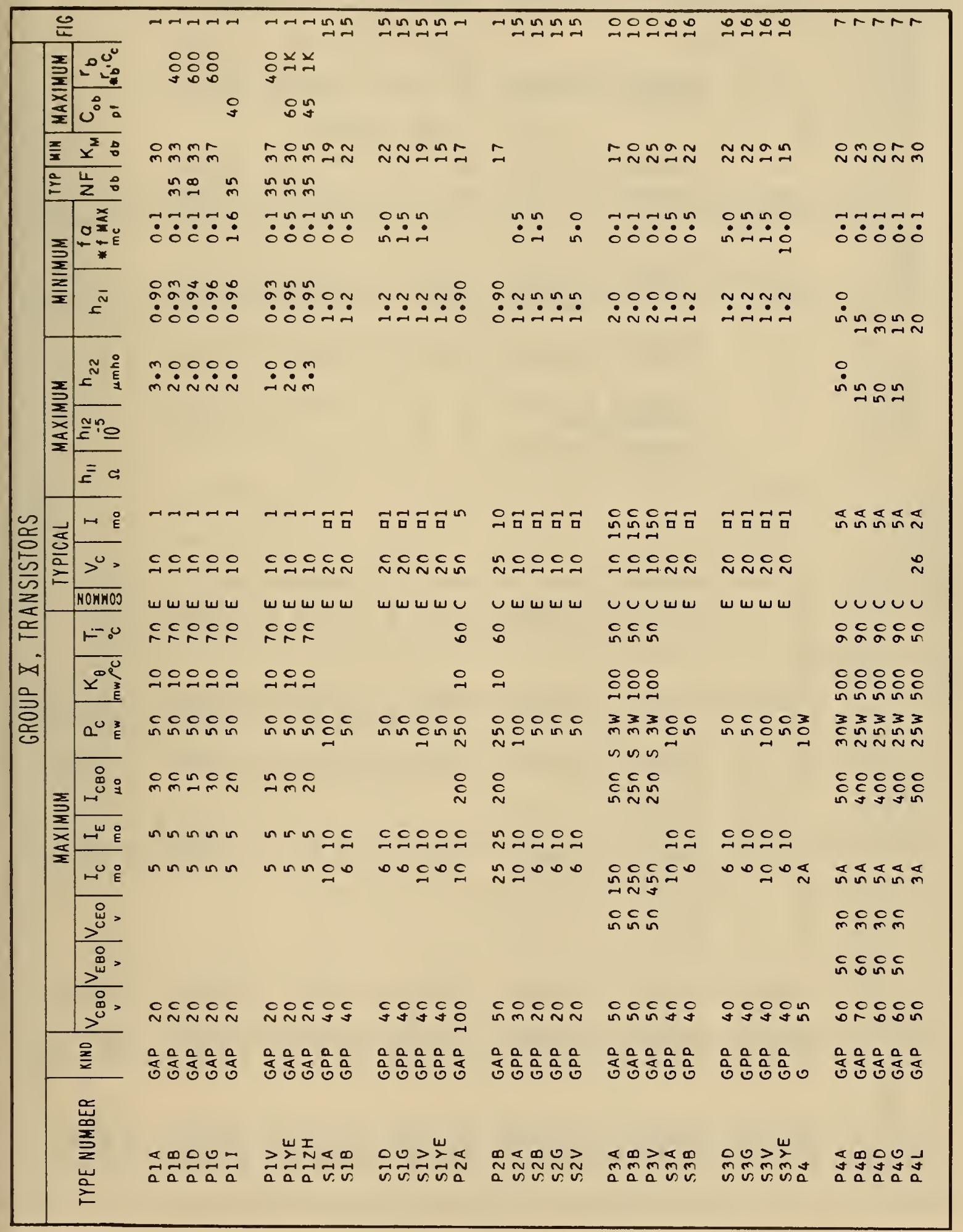




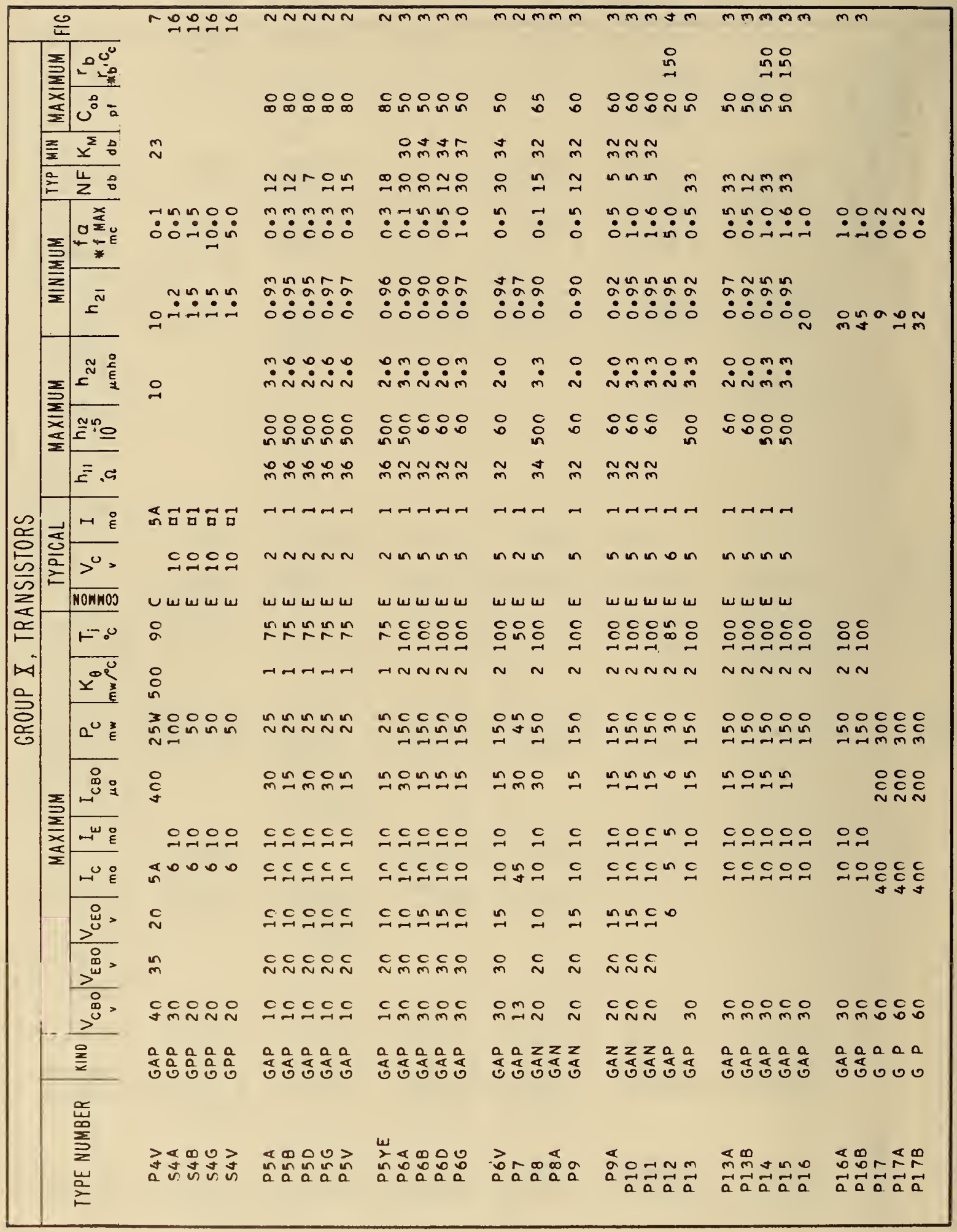




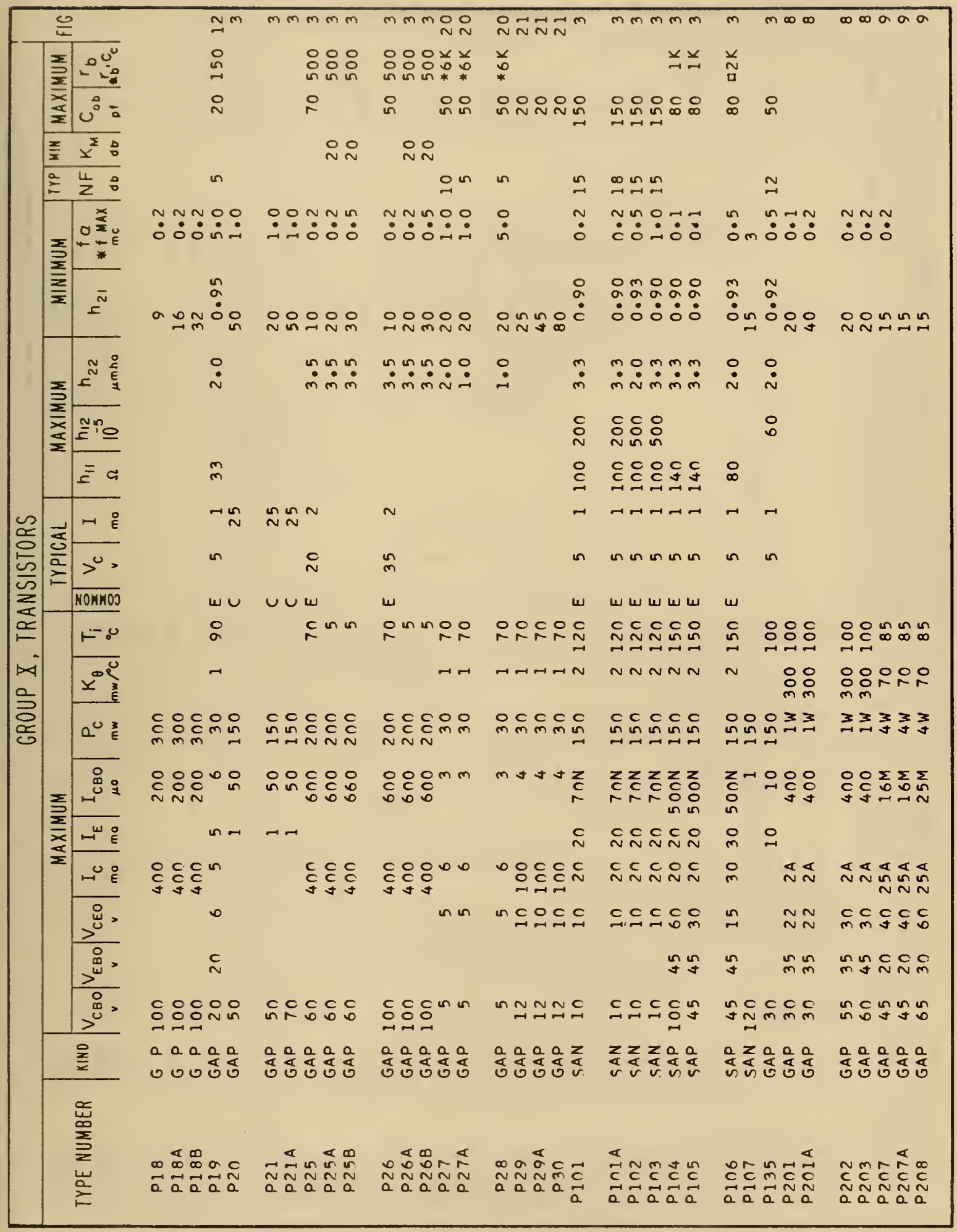




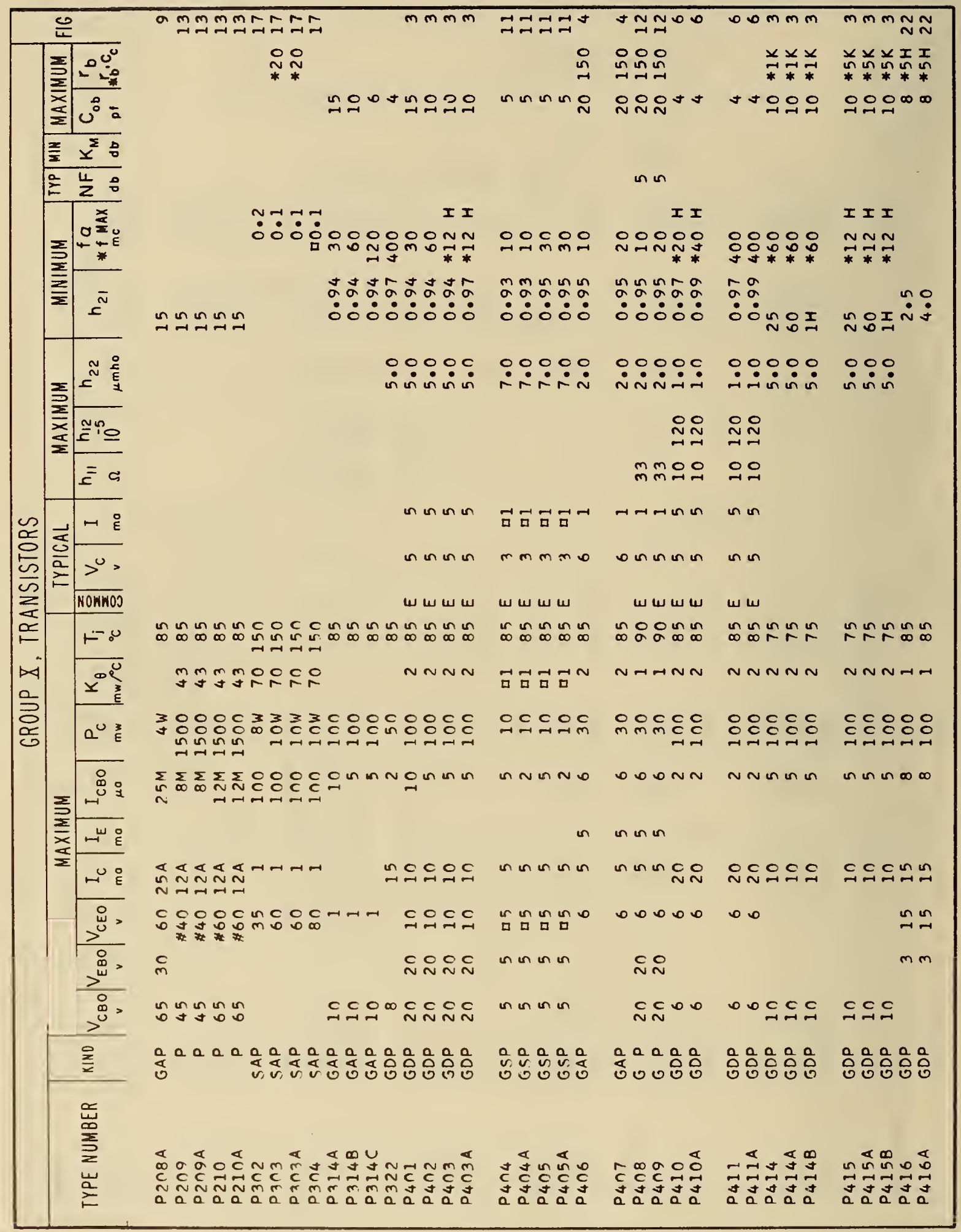




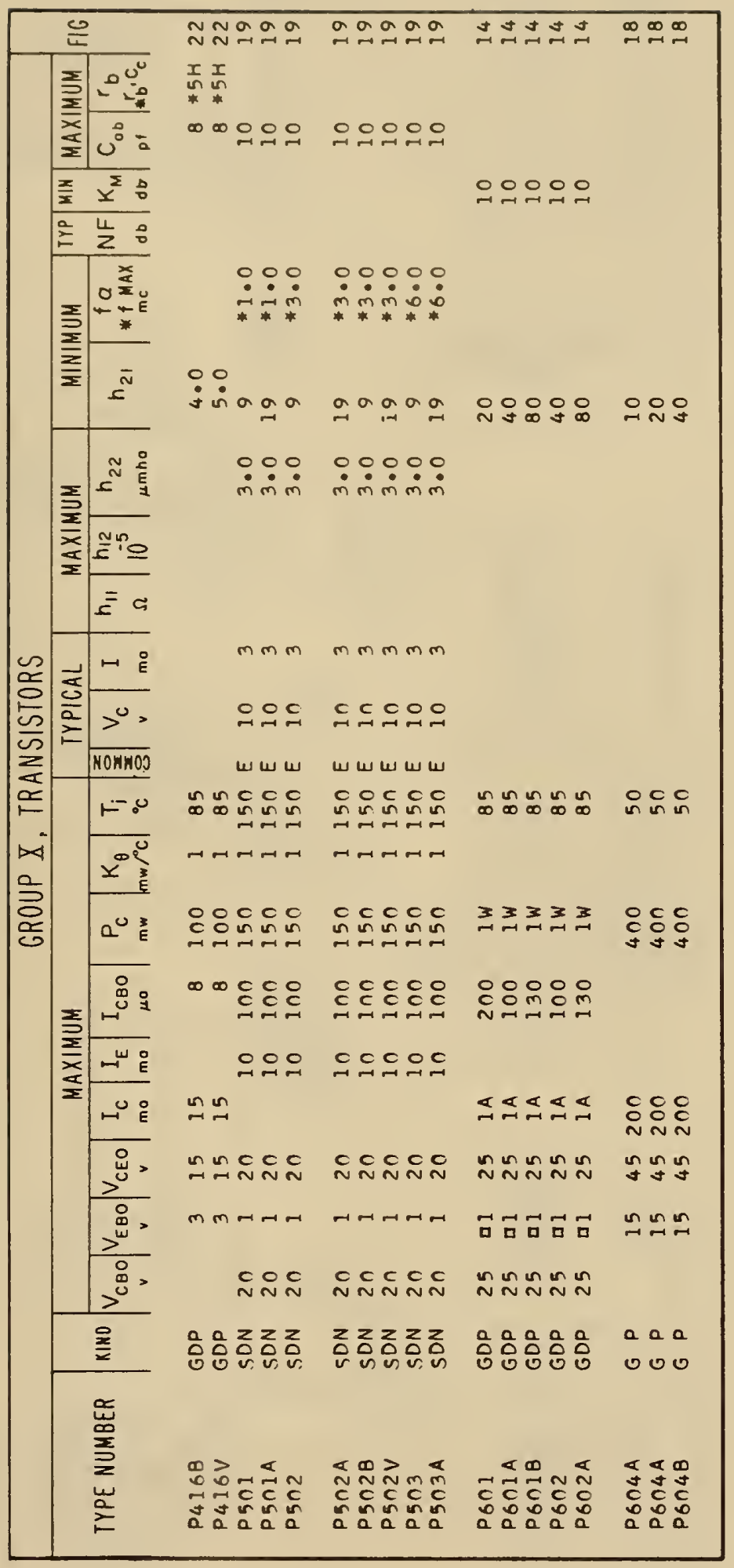



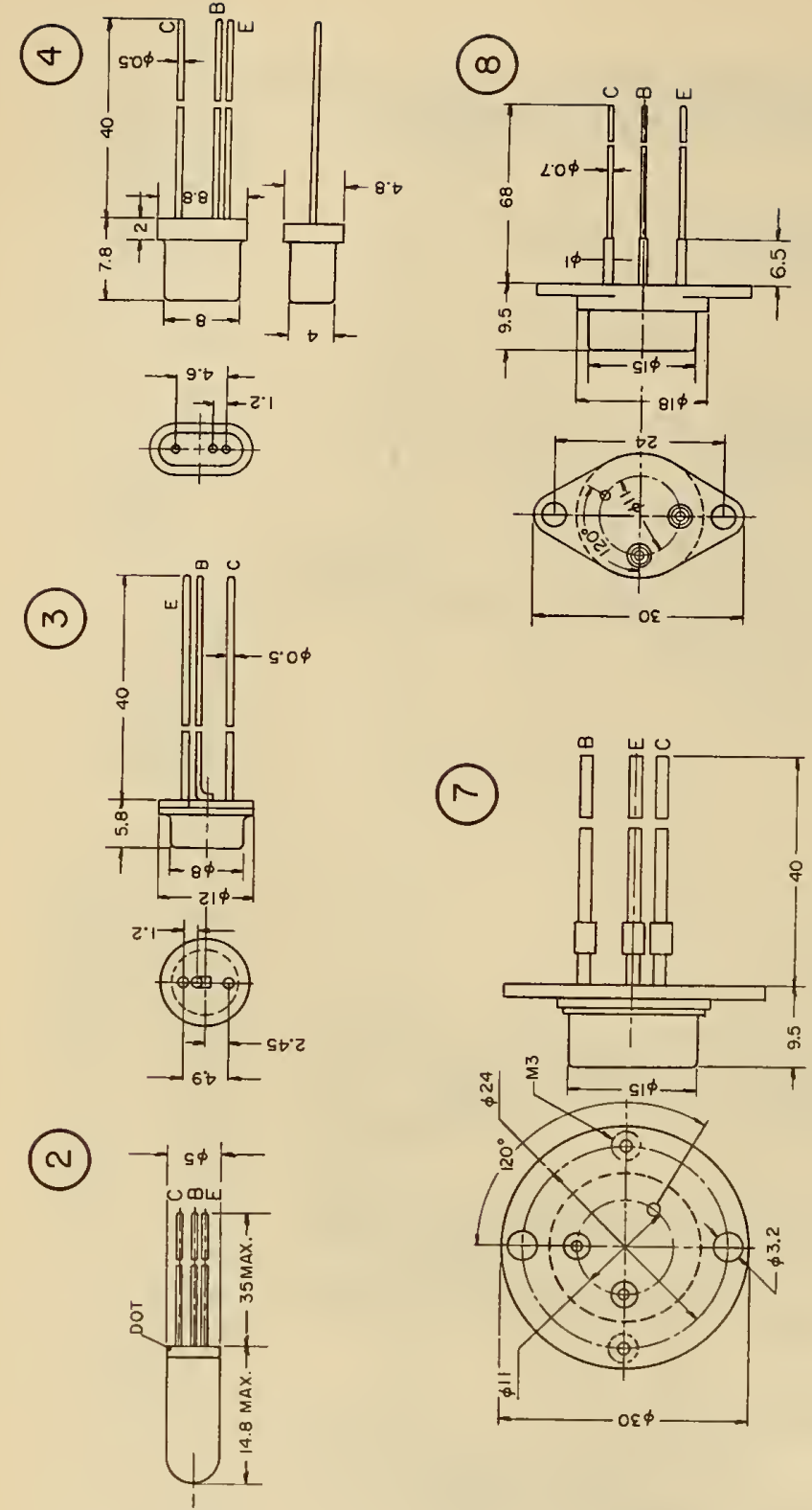

(

( )
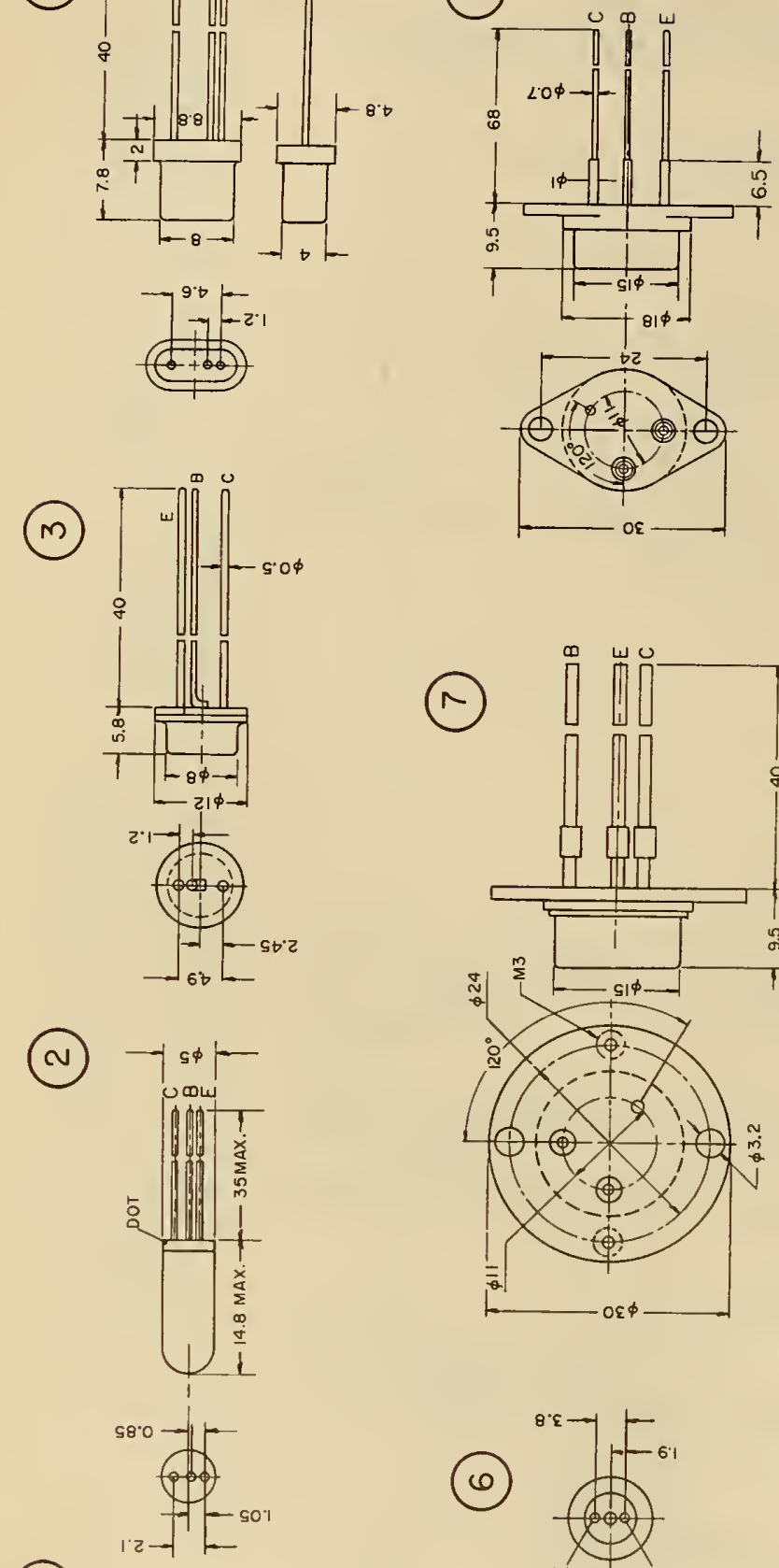

$\Theta$
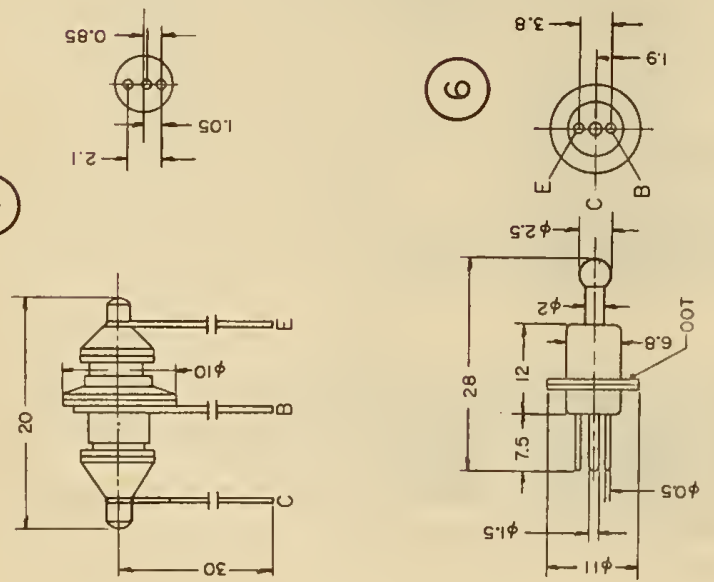
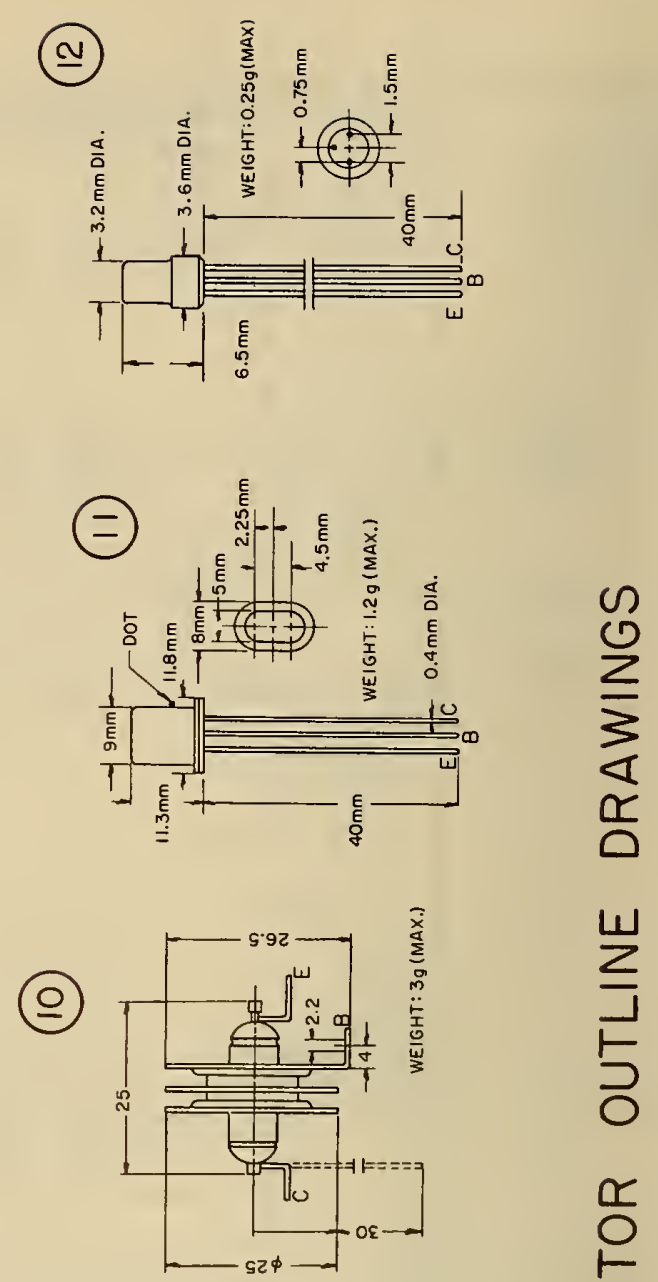

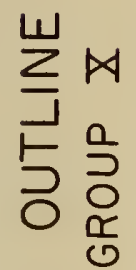

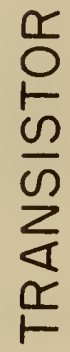

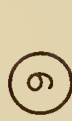




\begin{tabular}{|c|c|c|c|c|c|c|c|c|c|c|c|c|c|c|}
\hline \multicolumn{15}{|c|}{ GROUP XI, DIODES - RECTIFIERS } \\
\hline \multirow{2}{*}{\multicolumn{2}{|c|}{ TYPE NUMBER }} & \multirow[b]{2}{*}{ KIND } & \multirow[b]{2}{*}{ TYPE } & \multicolumn{3}{|c|}{ MAXIMUM } & \multicolumn{3}{|c|}{ MAXIMUM@25 $\mathrm{C}$} & \multicolumn{3}{|c|}{ MAXIMUM } & \multirow[b]{2}{*}{$\begin{array}{l}f_{\text {Max }} \\
\text { mc }\end{array}$} & \multirow[b]{2}{*}{ FIG } \\
\hline & & & & $\left|\begin{array}{c}I_{F} \\
\wp 25^{\circ} \mathrm{C} \\
\mathrm{mo}\end{array}\right|$ & $\begin{array}{c}T_{\text {Opr }} \\
{ }^{\circ} \mathrm{C}\end{array}$ & \begin{tabular}{|l|}
$I_{S}$ \\
$2 S^{\circ} \mathrm{C}$ \\
$A$
\end{tabular} & $\underset{v}{P I V}$ & {$\left[\begin{array}{c}E_{F} \\
\text { MIN. } E_{f}\end{array}\right]$} & $\begin{array}{l}I_{F} \\
\text { mo }\end{array}$ & $\left.I_{R}\right|_{\mu} ^{1}$ & $E_{r}$ e & $\begin{array}{l}\mathrm{T}^{0} \mathrm{C} \\
{ }^{\circ} \mathrm{C}\end{array}$ & & \\
\hline D $1 \mathrm{~A}$ & & REC & GEP & 16 & 70 & & 40 & 1.0 & 2 & 250 & 10 & & 150 & 1 \\
\hline$D 1 B$ & & REC & GEP & 16 & 70 & & 45 & 1.0 & 1 & 250 & 25 & & 150 & 1 \\
\hline 010 & & REC & GEP & 16 & 70 & & 110 & 1.0 & 2 & 250 & 75 & & 150 & 1 \\
\hline$D 1 G$ & & REC & GEP & 16 & 70 & & 75 & 1.0 & 5 & 250 & 50 & & 150 & 1 \\
\hline D $1 \mathrm{~V}$ & & REC & GEP & 25 & 70 & & 45 & 1.0 & 8 & 250 & 25 & & 150 & 1 \\
\hline DIYE & & REC & GEP & 12 & 70 & & 150 & 1.0 & 1 & 250 & 100 & & 1 bo & 1 \\
\hline $\mathrm{D} 1 \mathrm{ZH}$ & & REC & GEP & 12 & 70 & & 150 & 1.0 & 5 & 250 & 100 & & 150 & 1 \\
\hline DG-TS 1 & & REC & GEP & 16 & 70 & 口 1 & 50 & $* 1.0$ & 2 & 1000 & 50 & 20 & & 2 \\
\hline D2A & @ & REC & GEP & 50 & 70 & & 15 & 1.0 & so & 250 & 7 & & 150 & 4 \\
\hline $\mathrm{D} 2 \mathrm{~B}$ & @ & REC & GEP & 16 & 70 & & 45 & 1.0 & 10 & 250 & 10 & & 150 & 4 \\
\hline D2D & @ & REC & GEP & 16 & $7 c$ & & 100 & 1.0 & 10 & 250 & 50 & & 150 & 4 \\
\hline $\mathrm{D} 2 \mathrm{G}$ & @ & REC & GEP & 16 & 70 & & 100 & 1.0 & 5 & 250 & 50 & & 150 & 4 \\
\hline $\mathrm{D} 2 \mathrm{~K}$ & & REC & GEP & 16 & 70 & & 125 & 1.0 & 5 & 800 & 100 & & & 4 \\
\hline$D 2 M$ & & REC & GEP & 16 & 70 & & 125 & 1.0 & 5 & 250 & 100 & & & 4 \\
\hline $\mathrm{D} 2 \mathrm{~N}$ & & REC & GEP & 16 & 70 & & 170 & $1 \cdot 0$ & 5 & 800 & 150 & & & 4 \\
\hline D2P & & REC & GEP & 16 & 70 & & 180 & 1.0 & 5 & 250 & 150 & & & 4 \\
\hline $\mathrm{D} 2 \mathrm{R}$ & & REC & GEP & 16 & 70 & & 220 & 1.0 & 5 & 250 & 200 & & & 4 \\
\hline $02 \mathrm{~V}$ & @ & REC & GEP & 25 & 70 & & 60 & 1.0 & 10 & 250 & 30 & & 150 & 4 \\
\hline DZYE & @ & REC & GEP & 16 & 70 & & 150 & 1.0 & 10 & 250 & 100 & & 150 & 4 \\
\hline $\mathrm{D} 2 \mathrm{ZH}$ & @ & REC & GEP & $E$ & 70 & & 200 & 1.0 & 10 & 250 & 150 & & 150 & 4 \\
\hline DG-TS2 & & REC & GEP & 16 & 70 & $\square 1$ & 75 & $* 1.0$ & 4 & 500 & 50 & 20 & & 2 \\
\hline DG-TS3 & & REC & GEP & 25 & & & 50 & 1.0 & 2 & 100 & 50 & 20 & & 2 \\
\hline DG-TS4 & & $R E C$ & GEP & 16 & 70 & ㅁ 1 & 100 & $* 1.0$ & 2 & 800 & 75 & 20 & & 2 \\
\hline DG-TSS & & REC & GEP & 16 & 70 & $\square 1$ & 100 & $* 1.0$ & 1 & 250 & 75 & 20 & & 2 \\
\hline DG-TSG & & REC & GEP & 16 & 70 & ㅁ 1 & 125 & $* 1.0$ & 1 & 800 & 100 & 20 & & 2 \\
\hline D7A & & REC & GEP & 300 & 70 & & 50 & 0.5 & 300 & 300 & 50 & & & 5 \\
\hline$D 7 B$ & & REEC & GEP & 300 & 70 & & 100 & $c \cdot b$ & 300 & 300 & 100 & & $50 \mathrm{~K}$ & 5 \\
\hline D7D & & REC & GEP & $1<0$ & 70 & & 300 & 0.5 & 300 & 300 & 300 & & $50 K$ & 5 \\
\hline D7G & & REC C & GEP & 300 & 70 & & 200 & 0.5 & 300 & 300 & 200 & & bok & 5 \\
\hline D7V & & REC & GEP & 300 & 70 & & 150 & 0.5 & 300 & 300 & 150 & & $50 K$ & 5 \\
\hline D7YE & & REC & GEP & 100 & 70 & 25 & 350 & 0.5 & 300 & 300 & 350 & & $50 K$ & 5 \\
\hline $\mathrm{D} 7 \mathrm{ZH}$ & & REC & GEP & 100 & 70 & 25 & 400 & 0.5 & 300 & 300 & 400 & & $50 K$ & 5 \\
\hline DG-TS7 & & $R E C$ & GEP & 16 & 70 & ㅁ 1 & 125 & $* 1.0$ & 1 & 250 & 100 & 20 & & 2 \\
\hline DG-TS8 & & REC & GEP & 25 & 70 & ㅁ & 50 & $* 1.0$ & 10 & 500 & 30 & 20 & & 2 \\
\hline D9A & & REC & GEP & 25 & 70 & & 10 & $1 \cdot 0$ & 10 & 250 & 10 & & 40 & 1 \\
\hline D9B & & REC & GEP & 40 & 70 & & 10 & 1.0 & 90 & 250 & 10 & & 40 & 1 \\
\hline
\end{tabular}




\begin{tabular}{|c|c|c|c|c|c|c|c|c|c|c|c|c|c|c|}
\hline \multicolumn{15}{|c|}{ GROUP XI, DIODES - RECTIFIERS } \\
\hline \multirow{2}{*}{\multicolumn{2}{|c|}{ TYPE NUMBER }} & \multirow[b]{2}{*}{ KIND } & \multirow[b]{2}{*}{ TYPE } & \multicolumn{3}{|c|}{ MAXIMUM } & \multicolumn{3}{|c|}{ MAXIMUM@25 C } & \multicolumn{3}{|c|}{ MAXIMUM } & \multirow[b]{2}{*}{$\begin{array}{c}f_{\text {Max }} \\
m c\end{array}$} & \multirow[b]{2}{*}{ FIG } \\
\hline & & & & $\mid \begin{array}{c}I_{F} \\
\arg _{\mathrm{mn}}{ }^{\circ} \mathrm{C}\end{array}$ & $\left|\begin{array}{c}T_{O p r} \\
{ }^{\circ} \mathrm{C}\end{array}\right|$ & $\left|\begin{array}{c|}\mathrm{I}_{\mathrm{S}} \\
\operatorname{DS}_{\mathrm{A}} \mathrm{C}^{\circ} \mathrm{C}\end{array}\right|$ & $\underset{v}{P I V}$ & $\mid \begin{array}{c}E_{F} \\
\text { MIN. } \\
E_{F}\end{array}$ & $\begin{array}{l}I_{F} \\
\text { mo }\end{array}$ & $\left.I_{R 0}\right|_{\mu_{0}}$ & $E_{r} \stackrel{e}{q}$ & $\begin{array}{l}\mathrm{T}^{\circ} \mathrm{C} \\
{ }^{\circ} \mathrm{C}\end{array}$ & & \\
\hline D90 & & REC & GEP & 30 & 70 & & 30 & 1.0 & 60 & 250 & 30 & & 40 & 1 \\
\hline D9G & & REC & GEP & 25 & 70 & & 30 & 1.0 & 30 & 250 & 30 & & 40 & 1 \\
\hline 091 & & REC & GEP & 70 & 70 & & 30 & $* 1.0$ & 30 & 500 & 30 & 70 & 40 & 1 \\
\hline D9K & & REC & GEP & 60 & 70 & & 30 & $* 1.0$ & 60 & 400 & 30 & 70 & 40 & 1 \\
\hline DЯL & & REC & GEP & 30 & 70 & & 100 & $* 1.0$ & 30 & 250 & 100 & 70 & 40 & 1 \\
\hline D9V & & REC & GEP & 20 & 70 & & 30 & 1.0 & 10 & 250 & 30 & & 40 & 1 \\
\hline DQYE & & REC & GEP & 20 & $7 c$ & & 50 & 1.0 & 30 & 250 & 50 & & 40 & 1 \\
\hline D9ZH & & REC & GEP & 15 & 70 & & 100 & 1.0 & 10 & 250 & 100 & & 40 & 1 \\
\hline DG-TS9 & & REC & GE & 50 & 70 & $\square 1$ & 45 & $* 1.0$ & 10 & 100 & 10 & 20 & & 2 \\
\hline 010 & & REC & GEP & 50 & 70 & & 20 & 1.5 & 3 & 100 & 10 & 20 & 150 & 6 \\
\hline DIOA & & REC & GEP & 50 & 70 & & 20 & 1.5 & 5 & 200 & 10 & 20 & 150 & 6 \\
\hline DIOB & & REC & GEP & 50 & 70 & & 20 & 1.5 & 8 & 200 & 10 & 20 & 150 & 6 \\
\hline DGT S 10 & & REC & GE & 50 & 70 & D 1 & 45 & $* 1.0$ & 5 & 60 & 10 & 20 & & 2 \\
\hline D 11 & & REC & GEP & 70 & 70 & & 50 & 1.0 & 10 & 250 & 30 & & 600 & 7 \\
\hline D12 & & REC & GEP & 70 & 70 & & 75 & 1.0 & 5 & 250 & 50 & & 600 & 7 \\
\hline D12A & & REC & GEP & 70 & 70 & & 75 & 1.0 & 10 & 250 & 50 & & 600 & 7 \\
\hline DGTS12 & & REC & GEP & 16 & 70 & & 30 & 1.0 & & & & & & 2 \\
\hline 013 & & REC & GEP & 70 & 70 & & 100 & 1.0 & 10 & 250 & 75 & & 600 & 7 \\
\hline DGTS 13 & & REC & GEP & 16 & 70 & & 30 & 1.0 & & & & & & 2 \\
\hline 014 & & REC & GEP & 70 & 70 & & 125 & 1.0 & 3 & 250 & 100 & & 600 & 7 \\
\hline D $14 A$ & & REC & GEP & 70 & 70 & & 125 & 1.0 & 10 & 250 & 100 & & 600 & 7 \\
\hline DGTS 14 & @ & REC & GEP & 16 & 70 & & 50 & 1.0 & & & & & & 2 \\
\hline DGTS15 & @ & REC & GE & 50 & 70 & ㅁ 1 & 170 & $* 1.0$ & 1 & 800 & 150 & 20 & & 2 \\
\hline OGTS16 & @ & REC & $G E$ & 50 & 70 & $\square 1$ & 180 & $* 1.0$ & 1 & 250 & 150 & 20 & & 2 \\
\hline DGTS 17 & @ & REC & GE & 50 & 70 & ㅁ & 220 & $* 1.0$ & 1 & 800 & 200 & 20 & & 2 \\
\hline 021 & $@$ & REC & GEP & 16 & 70 & & 100 & 1.0 & 5 & 250 & 100 & 20 & 150 & 4 \\
\hline UGTS21 & @ & REC & GEA & 300 & 70 & $=5$ & 75 & $* 0.5$ & 300 & 500 & 50 & 20 & & 3 \\
\hline DGT 522 & @ & REC & GEA & 300 & 70 & 25 & 150 & $* 0.5$ & 300 & 500 & 100 & 20 & & 3 \\
\hline DGTS23 & ๑ & REC & GEA & 300 & 70 & 25 & 225 & $* 0.5$ & 300 & 500 & 150 & 20 & & 3 \\
\hline DGTS24 & ఏ & REC & GEA & 300 & 70 & 25 & 300 & $* 0.5$ & 300 & 500 & $2 \cap 0$ & 20 & & 3 \\
\hline DGT S25 & @ & REC & GEA & 100 & 70 & 25 & 450 & $* 0.3$ & 100 & 300 & 300 & 20 & & 3 \\
\hline DGTS26 & $@$ & REC & GEA & 100 & 70 & 25 & 525 & $* 0.3$ & 100 & 300 & 350 & 20 & & 3 \\
\hline DGTS27 & @ & REC & GEA & 100 & 70 & 25 & 600 & $* 0.3$ & 100 & 300 & 400 & 20 & & 3 \\
\hline 0101 & & REC & SIP & 50 & 150 & & 100 & *ż. 0 & 2 & 100 & 100 & 125 & 200 & 6 \\
\hline$D 1 C 1 A$ & & REC & SIP & 75 & ס 10 & & 100 & $* 1.0$ & 1 & 75 & 100 & 125 & 200 & 6 \\
\hline 0102 & & REC & SIP & 50 & 150 & & 75 & 2.0 & 2 & 100 & 75 & 125 & 200 & 6 \\
\hline
\end{tabular}




\begin{tabular}{|c|c|c|c|c|c|c|c|c|c|c|c|c|c|}
\hline & & & GRO & OUP & XI, D & IODES & - RECT & $|F| E R$ & & & & & \\
\hline & & & & $A X I M U$ & & MAXII & MUM@2 & $5^{\circ} \mathrm{C}$ & & AXIMUN & & & \\
\hline TYPE NUMBER & KIND & TYPE & $\mid$\begin{tabular}{c|}
$I_{F}$ \\
$\left(a 25^{\circ} \mathrm{C}\right.$ \\
$\mathrm{ma}$
\end{tabular} & $\begin{array}{l}T_{\text {Opr }} \\
{ }^{\circ} \mathrm{C}\end{array}$ & $\mid \begin{array}{c}I_{S} \\
ه 25_{A} \mathrm{C}\end{array}$ & $\underset{v}{P I V}$ & $\mid \begin{array}{c}E_{F} \\
\text { MIN. } \\
E_{F}\end{array}$ & $\begin{array}{l}I_{F} \\
m o\end{array}$ & $I_{R} \underset{a}{a}$ & $E_{i}$ & ${ }^{\circ} \mathrm{C}$ & $\begin{array}{l}f_{\text {Max }} \\
\text { mc }\end{array}$ & FIG \\
\hline D $102 A$ & REC & S1P & 75 & 150 & & 75 & 1.0 & 1 & 100 & 75 & 125 & 200 & 6 \\
\hline D 103 & REC & S1P & 50 & 150 & & 30 & 2.0 & 2 & 100 & 30 & 125 & 200 & 6 \\
\hline D $103 A$ & REC & S1P & 75 & 150 & & 30 & 1.0 & 1 & 100 & 30 & 125 & 200 & 6 \\
\hline 0104 & REC & S1P & 50 & 150 & & 100 & 2.0 & 2 & 100 & 100 & 125 & & b \\
\hline D $104 \mathrm{~A}$ & REC & S1P & 75 & 150 & & 100 & 1.0 & 1 & 75 & 100 & 125 & & 8 \\
\hline 0105 & REC & $S 1 P$ & 50 & 150 & & 75 & 2.0 & 2 & 100 & 75 & 125 & & 8 \\
\hline D $105 A$ & RFC & S1P & 75 & ס & & 75 & 1.0 & 1 & 100 & 75 & 125 & & 8 \\
\hline 0106 & REC & S1P & 50 & 150 & & 30 & 2.0 & 2 & 100 & 30 & 125 & & 8 \\
\hline D $106 \mathrm{~A}$ & REC & S $1 P$ & 75 & $1 \supset 0$ & & 30 & 1.0 & 1 & 100 & 30 & 125 & & 8 \\
\hline$D 201 \mathrm{~A}$ & REC & S 1 & 200 & 125 & & 25 & 1.5 & & & & & & \\
\hline $\begin{array}{l}D 201 B \\
02010\end{array}$ & $\begin{array}{l}\text { REC } \\
\text { REC }\end{array}$ & $\begin{array}{l}\text { S1 } \\
\text { S1 }\end{array}$ & $\begin{array}{l}200 \\
400\end{array}$ & $\begin{array}{l}125 \\
125\end{array}$ & & $\begin{array}{r}50 \\
100\end{array}$ & $\begin{array}{l}1.5 \\
2.0\end{array}$ & & & & & & \\
\hline $0201 G$ & REC & s1 & 200 & 125 & & 100 & 1.5 & & & & & & \\
\hline D201TS & REC & S1 & 400 & 125 & & 200 & 2.0 & & & & & & \\
\hline $0201 \mathrm{~V}$ & REC & S1 & 400 & 125 & & 50 & 2.0 & & & & & & \\
\hline DZUIYE & REC & S 1 & 200 & 125 & & 200 & 2.0 & & & & & & \\
\hline $0201 \mathrm{ZH}$ & REC & S1 & $4 \cup 0$ & 125 & & 200 & 2.0 & 400 & 500 & 200 & & & \\
\hline 0202 & REC & SIA & 400 & 150 & & 100 & 1.5 & 400 & 500 & 100 & 125 & 0.1 & 9 \\
\hline 0203 & REC & $S 1 A$ & 400 & 150 & & 200 & 1.5 & 400 & 500 & 200 & 125 & 0.1 & 9 \\
\hline 0204 & REC & SIA & 400 & 150 & & 300 & 1.5 & 400 & 500 & 300 & 125 & 0.1 & $\varphi$ \\
\hline 0205 & REC & $S I A$ & 400 & סכ: 1 & & 400 & 1.5 & 400 & 500 & 400 & 125 & 0.1 & $\vartheta$ \\
\hline 0206 & REC & SIA & 100 & 125 & & 100 & 1.0 & 100 & 100 & 100 & 125 & 0.1 & 10 \\
\hline D207 & REC & S1A & 100 & 125 & & 200 & 1.0 & 100 & 100 & 200 & 125 & 0.1 & 10 \\
\hline 0208 & REC & SIA & 100 & 125 & & 300 & 1.0 & 100 & 100 & 300 & 125 & 0.1 & 10 \\
\hline 0209 & REC & SIA & 100 & 125 & & 400 & 1.0 & 100 & 100 & 400 & 125 & 0.1 & 10 \\
\hline 0210 & REC & S1A & 100 & 125 & & 500 & 1.0 & 100 & 100 & 500 & 125 & 0.1 & 10 \\
\hline D211 & REC & SIA & 100 & 125 & & 600 & 1.0 & 100 & 100 & 600 & 125 & 0.1 & 10 \\
\hline 0302 & REC & GEA & $1 \mathrm{~A}$ & 70 & & 200 & 0.25 & $1 \mathrm{~A}$ & 1000 & 200 & 20 & $50 K$ & 11 \\
\hline 0303 & REC & GEA & $3 A$ & 70 & & 150 & 0.3 & $3 A$ & 1000 & 150 & 20 & $50 \mathrm{~K}$ & 11 \\
\hline D 304 & RE- $C$ & GEA & $S A$ & 70 & & 100 & 0.3 & $\triangle A$ & 3000 & 150 & 20 & $50 K$ & 11 \\
\hline D305 & REC & GEA & $10 \mathrm{~A}$ & 70 & & 50 & 0.35 & $10 A$ & 3000 & 50 & 20 & 占: & 11 \\
\hline D1001 & REC & $G E$ & 100 & 80 & & 2000 & 6.5 & 100 & 150 & 2000 & & 口0.1 & 12 \\
\hline D1001A & REC & GE & 100 & 80 & & 1000 & $3 \cdot 5$ & 100 & 150 & 1000 & & 口O. 1 & 12 \\
\hline D1002 & REC & $G E$ & 300 & 80 & & 2000 & $7 \cdot 5$ & 300 & 300 & 2000 & & प्. 1 & 13 \\
\hline DIO02A & REC & $G E$ & 300 & $\varnothing 0$ & & 1000 & 4.0 & 300 & 300 & 1000 & & प0.1 1 & 13 \\
\hline D1003A & RE:C & $G E$ & 300 & 80 & & 500 & $2 \cdot 0$ & 300 & 300 & 500 & & $\square 0.1$ & 12 \\
\hline
\end{tabular}




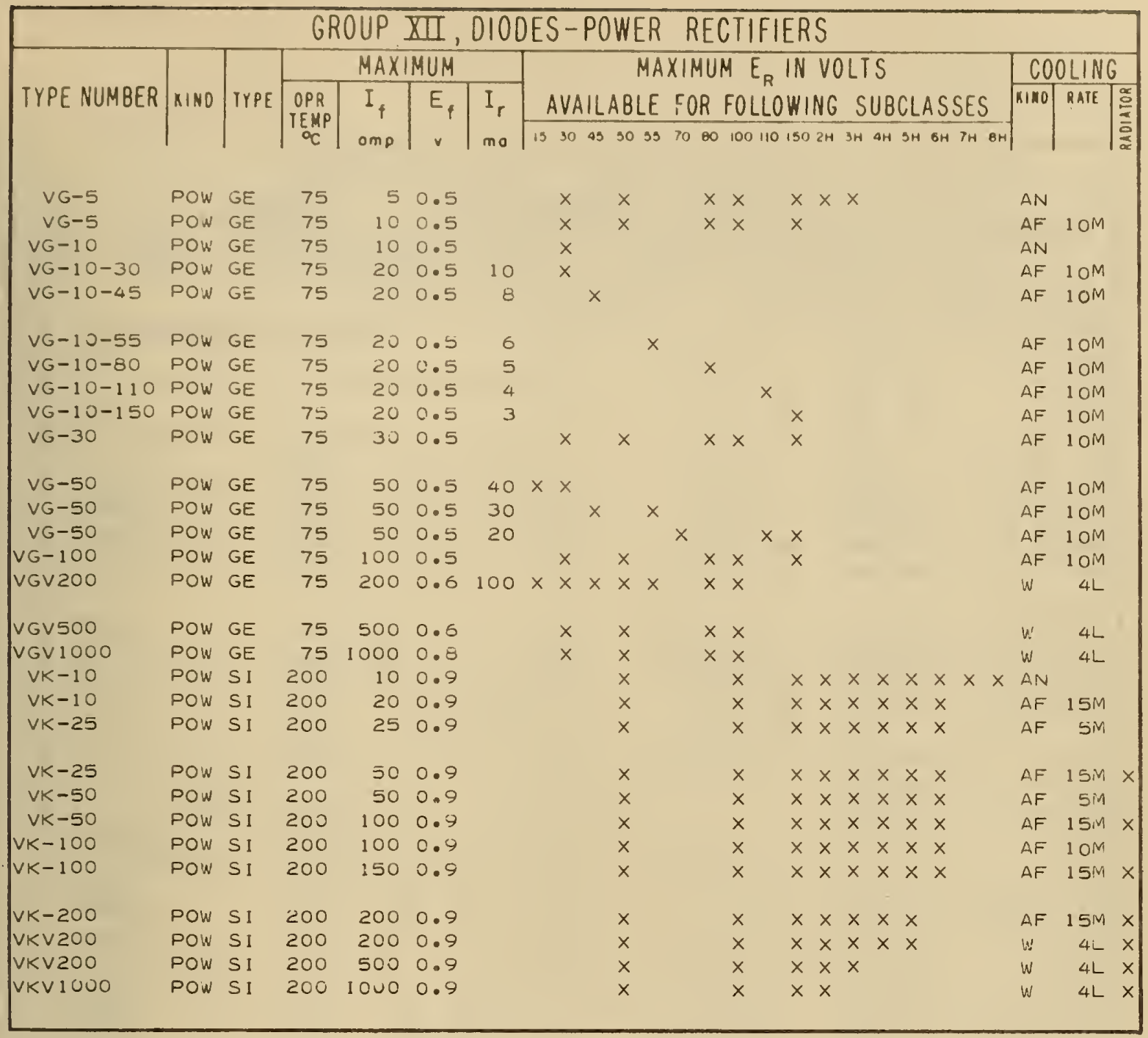

\begin{tabular}{|c|c|c|c|c|c|c|c|c|c|c|c|c|}
\hline \multicolumn{13}{|c|}{ GROUP XIII, DIODES - REGULATORS } \\
\hline \multirow[b]{2}{*}{ TYPE NUMBER } & \multirow[b]{2}{*}{ KIND } & \multirow[b]{2}{*}{ TYPE } & \multicolumn{3}{|c|}{ MAXIMUM } & \multicolumn{3}{|c|}{ TYPICAL } & \multirow{2}{*}{$\begin{array}{c}\text { MAX } \\
Z \\
\Omega\end{array}$} & \multirow[b]{2}{*}{$\begin{array}{l}\mathrm{TC} \\
\% /{ }^{\circ} \mathrm{C}\end{array}$} & \multirow[b]{2}{*}{$\left|\begin{array}{c}\mathrm{K}_{\theta} \\
\mathrm{mw} /{ }^{\circ} \mathrm{C}\end{array}\right|$} & \multirow[b]{2}{*}{ fig } \\
\hline & & & $\begin{array}{l}I_{Z} \\
m o\end{array}$ & $\begin{array}{c}T_{\text {Opr }} \\
c\end{array}$ & $\underset{\mathrm{mw}}{\mathrm{P}_{\mathrm{z}}}$ & $E_{z}$ & $\left|\begin{array}{c}\Delta E_{z} \\
\%\end{array}\right|$ & $\begin{array}{l}I_{z} \\
m o\end{array}$ & & & & \\
\hline D6 & REG & SI & 18 & 150 & 125 & 6.5 & & 5.0 & 10 & .03 & & \\
\hline 07 & REG & SI & 18 & 150 & 125 & 7.5 & & 5.0 & 10 & .06 & & \\
\hline 08 & REG & SI & 14 & 150 & 125 & $8 \cdot 5$ & & 5.0 & 10 & .07 & & \\
\hline 0808 & REG & SI & 33 & 125 & 280 & 7.7 & 10 & 5.0 & 6 & .07 & 3 & 14 \\
\hline D809 & REG & SI & 29 & 125 & 280 & 8.7 & 10 & 5.0 & 10 & .08 & 3 & 14 \\
\hline D810 & REG & SI & 26 & 125 & 280 & 9.7 & 10 & 5.0 & 12 & .09 & 3 & 14 \\
\hline D811 & REG & SI & 23 & 125 & 280 & 11.0 & 10 & 5.0 & 15 & .095 & 3 & 14 \\
\hline 0813 & REG & SI & 20 & 125 & 280 & 12.7 & 10 & 5.0 & 18 & .095 & 3 & 14 \\
\hline
\end{tabular}




\begin{tabular}{|c|c|c|c|c|c|c|c|c|c|c|c|c|c|c|c|}
\hline \multicolumn{16}{|c|}{ GROUP XV, DIODES - MIXER \& DETECTOR } \\
\hline \multirow[b]{2}{*}{ TYPE NUMBER } & \multirow[b]{2}{*}{ KIND } & \multirow[b]{2}{*}{ TYPE } & \multicolumn{3}{|c|}{ MAXIMUM } & \multicolumn{2}{|c|}{ FREOUENCY } & \multirow{2}{*}{$\frac{\text { MIN. }}{F M}$} & \multicolumn{5}{|c|}{ MAXIMUM } & \multirow{2}{*}{$\begin{array}{l}P_{80} \\
\text { ergs } \\
\mathrm{mw}^{\mathrm{w}}\end{array}$} & \multirow{2}{*}{ FIG } \\
\hline & & & $\begin{array}{l}Z_{v} \\
k \Omega\end{array}$ & $\left|\begin{array}{c|}T_{0 p r} \\
{ }_{0 p}^{\circ} \mathrm{C}\end{array}\right|$ & $\left.\right|_{\Omega} ^{Z_{I F}}$ & $\begin{array}{l}M / N \\
G_{C}\end{array}$ & $\begin{array}{c}\text { MAX } \\
G_{C}\end{array}$ & & NR & VSWR & $\begin{array}{l}\text { NF } \\
d b\end{array}$ & $\underset{d D}{\text { LC }}$ & $\begin{array}{c}\text { POWER } \\
\text { LEVEL } \\
\text { mw }\end{array}$ & & \\
\hline$D G-S 1$ & MIX & GEP & & 70 & & & $3 \cdot 1$ & & & 3.5 & 3.0 & 8.5 & 800 & 0.1 & 15 \\
\hline$D K-11$ & $\operatorname{Mix}$ & SI & & 70 & & & 3.1 & & & & & & & $* 200$ & 17 \\
\hline$D K-S 1$ & MIX & SIP & & 70 & & & $3 \cdot 1$ & & & 3.0 & 2.7 & 8.5 & 800 & 0.1 & 17 \\
\hline$D K-V 1$ & DET & GE & 15 & 70 & & & $3 \cdot 1$ & & & & & & & $* 200$ & 15 \\
\hline DL-S1 & MIX & S1P & & 70 & & & $3 \cdot 1$ & & & 3.5 & 3.0 & 8.5 & 800 & 口0.1 & \\
\hline DG-S2 & MIX & GEP & & 70 & & & 3.1 & & & 3.0 & 3.0 & 6.5 & 800 & 0.1 & 15 \\
\hline$D K-12$ & MIX & SI & & 70 & & & 9.4 & & & & & & & *200 & 17 \\
\hline$D K-S 2$ & MIX & SIP & & 70 & & & 3.1 & & & 3.0 & 2.0 & 6.5 & 500 & 口0.1 & 17 \\
\hline$D K-v 2$ & DET & GE & 10 & 70 & & & 3.1 & & & & & & & $* 100$ & 15 \\
\hline $\mathrm{DL}-\mathrm{S2}$ & MIX & SIP & & 70 & & & 3.1 & & & 3.0 & 3.0 & 6.5 & 800 & 口0.1 & \\
\hline D3A & DET & s1 & & 70 & & & & & & 2.5 & & & & $* 300$ & 15 \\
\hline D $3 B$ & DET & s1 & & 70 & & & 3.1 & & & 2.5 & & & & $* 300$ & 15 \\
\hline$D G-53$ & MIX & GEP & & 70 & & & 9.4 & & & 3.5 & 3.0 & 8.5 & 800 & 0.1 & 15 \\
\hline$D K-53$ & MIX & SIP & & 70 & & & 9.4 & & & 3.0 & 2.7 & 8.5 & 500 & $\square 0.1$ & 17 \\
\hline$D K-V 3$ & DET & GE & 15 & 70 & & & 9.4 & & & & & & & *200 & 15 \\
\hline$D L-53$ & MIX & SIP & & 70 & & & 9.4 & & & 3.5 & 3.0 & $8 \cdot 5$ & 800 & व०.1 & \\
\hline$D G-54$ & MIX & GEP & & 70 & & & 9.4 & & & 3.0 & 3.0 & 6.5 & 800 & 0.1 & $1 b$ \\
\hline$D K-54$ & $M 1 X$ & SIP & & 70 & & & 9.4 & & & 2.5 & $2 \cdot 7$ & 6.5 & & 0.3 & 17 \\
\hline$D K-V 4$ & DET & $G E$ & 10 & 70 & & & 9.4 & & & & & & & $* 100$ & 15 \\
\hline $\mathrm{DL}-54$ & MIX & S1P & & 70 & & & 9.4 & & & 3.0 & 3.0 & 6.5 & 800 & 口0.1 & \\
\hline$D K-V 5$ & DET & GE & 10 & 70 & & & 3.1 & & & & & & & $* 200$ & 17 \\
\hline$D K-v G$ & DET & GE & 25 & 70 & & & $3 \cdot 1$ & & & & & & & $* 200$ & 17 \\
\hline$D K-S 7$ & $M 1 X$ & SIP & & 70 & & & 9.4 & & & 2.0 & $2 \cdot 0$ & $7 \cdot 0$ & 500 & ㅁ. 1 & 15 \\
\hline$D K-V 7$ & DET & $G E$ & 10 & 70 & & & 9.4 & & & & & & & $* 200$ & 17 \\
\hline 0401 & MOD & GE & & 50 & & & & & & & & 13.0 & & *300 & 16 \\
\hline D403A & MIX & GE & & 100 & 700 & & 3.0 & & 3.0 & & & 9.0 & & 0.3 & 15 \\
\hline $0403 B$ & MIX & GE & & 100 & 600 & 2.5 & 9.4 & & 3.0 & & & $8 \cdot 5$ & & 0.3 & 15 \\
\hline $0403 \mathrm{~V}$ & MIX & GE & & 100 & 600 & & 3.0 & & & & 13.0 & 9.0 & & 0.3 & د5 \\
\hline 0405 & DET & SI & & & 400 & & 10.0 & & & & & $7 \cdot 0$ & 800 & 0.6 & 18 \\
\hline D405A & DET & SI & & & 350 & & 10.0 & & & & & $6 \cdot 0$ & 800 & 1.0 & 18 \\
\hline D405AP & DET & SI & & & 350 & & 10.0 & & & & & 6.0 & 800 & $1 \cdot 0$ & 18 \\
\hline D405B & DET & SI & & & 330 & & 10.0 & & & & & 8.0 & 800 & 1.0 & 18 \\
\hline $0405 B P$ & DET & SI & & & 330 & & 10.0 & & & & & 8.0 & 800 & $1 \cdot 0$ & 18 \\
\hline $0602 A$ & VID & GE & & 86 & 600 & & 10.0 & 15 & & 3.2 & & & 500 & & 15 \\
\hline 06028 & VID & GE & & 85 & 600 & & 10.0 & 20 & & 3.2 & & & 500 & & 15 \\
\hline D602V & DET & GE & & 85 & & & 9.4 & & & & & & & & 15 \\
\hline D603 & V1D & SI & & & 900 & & 5.0 & & & 2.0 & & & $2 H$ & & 17 \\
\hline
\end{tabular}




\begin{tabular}{|c|c|c|c|c|c|c|c|c|c|c|}
\hline \multicolumn{11}{|c|}{ GROUP XV, DIODES-PHOTOCONDUCTIVE } \\
\hline \multirow{3}{*}{ TYPE NUMBER } & \multirow{3}{*}{ KINO } & \multirow{3}{*}{$\begin{array}{l}\text { CATH } \\
\text { AREA } \\
\mathrm{mm}^{2}\end{array}$} & \multirow{3}{*}{$\begin{array}{l}\text { MIN.OARK } \\
\text { RES. } \\
\text { meg } \Omega\end{array}$} & \multirow{3}{*}{$\begin{array}{c}\text { MAX. } \\
\text { WORKING } \\
v\end{array}$} & \multirow{3}{*}{$\begin{array}{l}\text { SENS. } \\
\mu \mathrm{o} / \mathrm{Im}\end{array}$} & \multirow{3}{*}{$\begin{array}{c}\text { T.C. } \\
\text { CURRENT } \\
\%\end{array}$} & \multicolumn{2}{|c|}{ SPEC.SENS. } & \multicolumn{2}{|c|}{ TEMP. } \\
\hline & & & & & & & MAX & CUtOFF & MIN & $\operatorname{MAX}$ \\
\hline & & & & & & & $\mu$ & $\mu$ & $(-)^{8}$ & $+10^{\circ} \mathrm{C}$ \\
\hline$F S-A G$ & PHC & 24 & 0.04 & 15 & 500 & 1.5 & $2 \cdot 1$ & 2.7 & 60 & 60 \\
\hline$F S-A O$ & PHC & 24 & 0.04 & 15 & 500 & $1 \cdot 5$ & $2 \cdot 1$ & $2 \cdot 7$ & 60 & 60 \\
\hline$F S-A V$ & PHC & 96 & 0.01 & 100 & 500 & 1.5 & $2 \cdot 1$ & 2.7 & 60 & 60 \\
\hline$F S-D O$ & $\mathrm{PHC}$ & 25 & 20.0 & 200 & $20 M$ & $2 \cdot 0$ & 0.75 & $1 \cdot 2$ & 60 & 40 \\
\hline$F S-K G$ & PHC & 25 & $3 \cdot 3$ & & 6000 & 0.2 & 0.64 & 0.9 & 60 & 80 \\
\hline FS - KO & PHC & 25 & $3 \cdot 3$ & 300 & 1200 & 0.12 & 0.52 & 0.9 & 60 & 80 \\
\hline$F S-K V$ & PHC & 50 & 1.6 & 200 & 6000 & 0.2 & 0.64 & 0.9 & 60 & 80 \\
\hline$F S-A 1$ & PHC & 24 & 0.04 & 15 & 500 & 1.5 & $2 \cdot 1$ & $2 \cdot 7$ & 60 & 60 \\
\hline$F S-D 1$ & $\mathrm{PHC}$ & 25 & $20 \cdot 0$ & 200 & $20 M$ & $2 \cdot 0$ & 0.75 & $1 \cdot 2$ & 60 & 40 \\
\hline$F S-K 1$ & PHC & 25 & $3 \cdot 3$ & 400 & 6000 & 0.2 & 0.64 & 0.9 & & \\
\hline$F S-2 A$ & PHC & 9 & 0.3 & 17.5 & & & 0.7 & $3 \cdot 5$ & 60 & 40 \\
\hline$F S-B 2$ & PHC & 121 & 0.2 & 50 & & & & & & \\
\hline$F s-K 2$ & $\mathrm{PHC}$ & 25 & $3 \cdot 3$ & 300 & 1200 & 0.12 & 0.52 & 0.9 & 60 & 80 \\
\hline$F S-3 A$ & PHC & 52 & $2 \cdot 0$ & 10 & & & 0.7 & 3.5 & 60 & 40 \\
\hline$F S-K 3$ & PHC & 25 & $3 \cdot 3$ & 300 & 1200 & 0.12 & 0.52 & 0.9 & 60 & 80 \\
\hline $\mathrm{FS}-\mathrm{A}_{4}$ & PHC & 24 & 0.04 & 15 & 500 & $1 \cdot 5$ & $2 \cdot 1$ & 2.7 & 60 & 60 \\
\hline$F S-K 4$ & PHC & 24 & $2 \cdot 0$ & 300 & 6000 & 0.2 & 0.64 & 0.9 & 60 & 80 \\
\hline$F S-K 5$ & PHC & 7 & $10 \cdot 0$ & 300 & 3000 & 0.2 & 0.64 & 0.9 & 60 & 80 \\
\hline$F S-A G$ & PHC & 115 & 0.05 & 30 & 500 & 1.5 & $2 \cdot 1$ & $2 \cdot 7$ & 60 & 60 \\
\hline FS-D6 & PHC & 115 & $20 \cdot 0$ & 200 & $20 M$ & $2 \cdot 0$ & 0.75 & 1.2 & 60 & 40 \\
\hline$F S-K 6$ & PHC & 115 & $3 \cdot 3$ & 300 & 3000 & 0.2 & 0.64 & c.9 & 60 & 80 \\
\hline$F S-K 7$ & PHC & 200 & 0.05 & 100 & 3500 & 0.2 & 0.64 & 0.9 & 60 & 80 \\
\hline$F S-K 8$ & PHC & 15 & 10.0 & 300 & 1600 & 0.2 & 0.64 & 0.9 & 60 & 80 \\
\hline
\end{tabular}



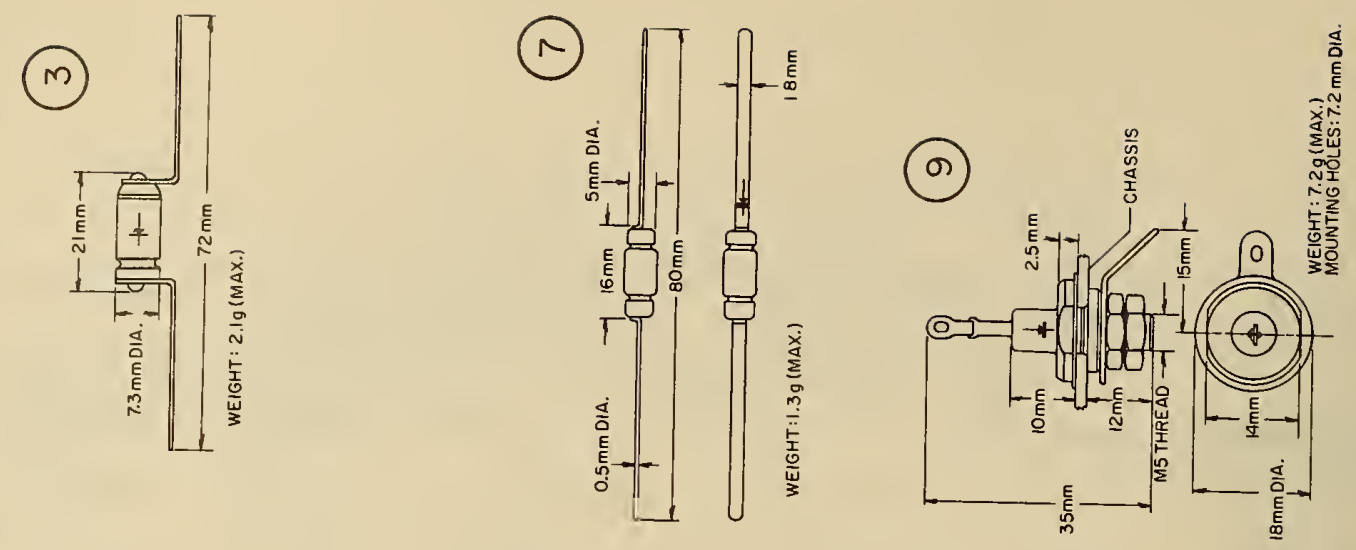

(a)
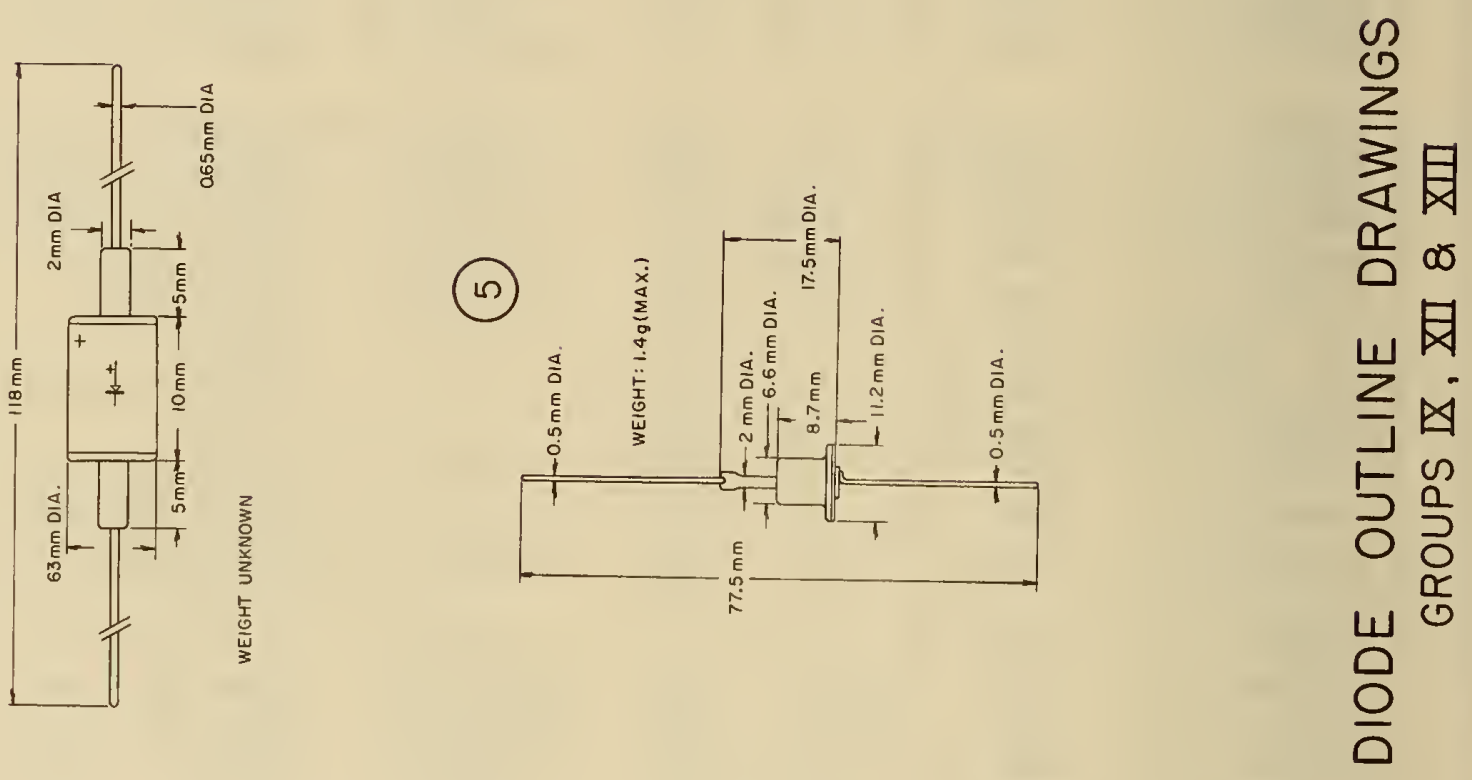

$\Theta$
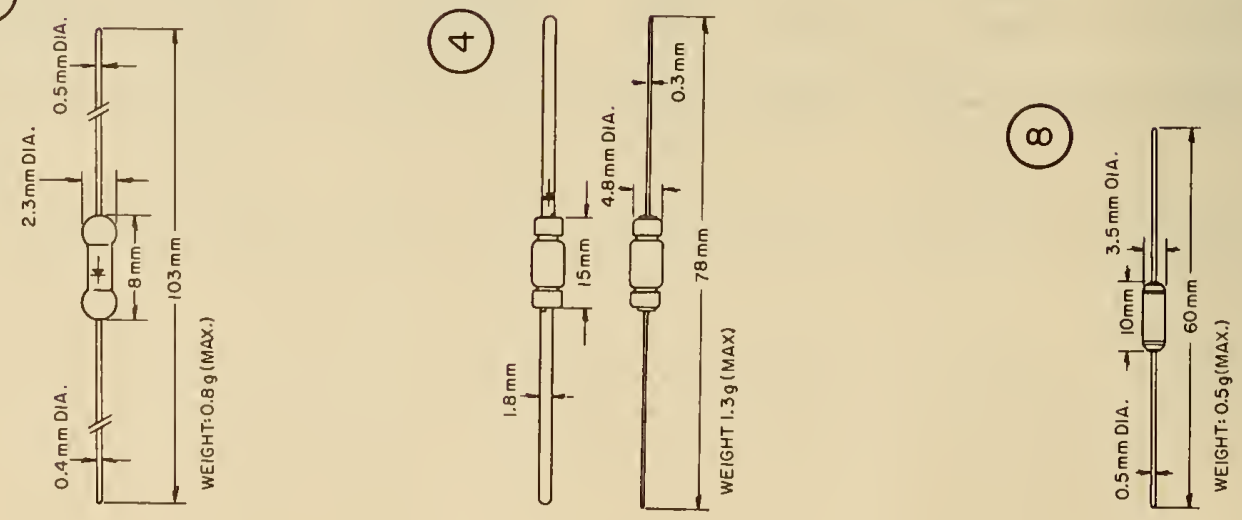
(ㅇ)

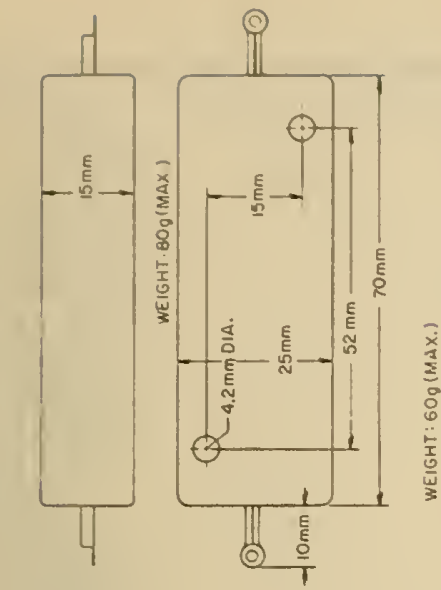

().

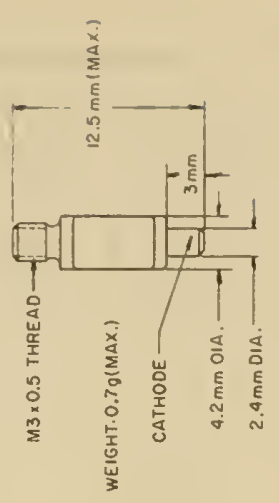

$(\ominus)$

$\Theta$

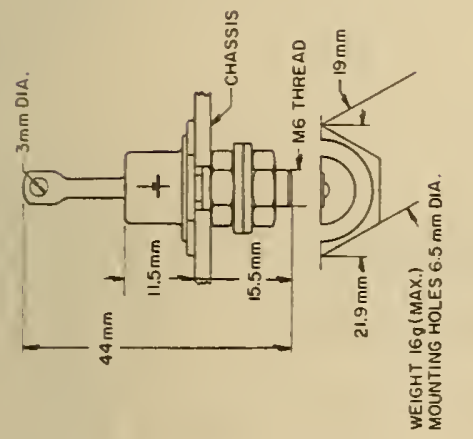

(ㅇ)

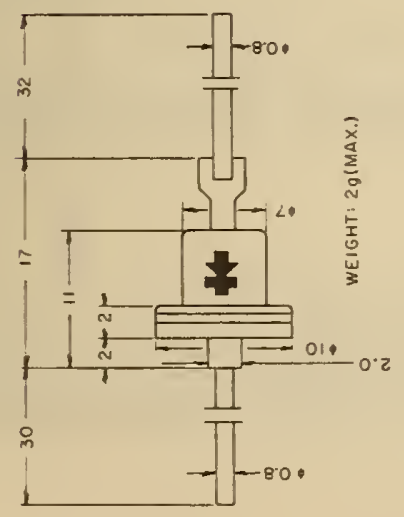

().

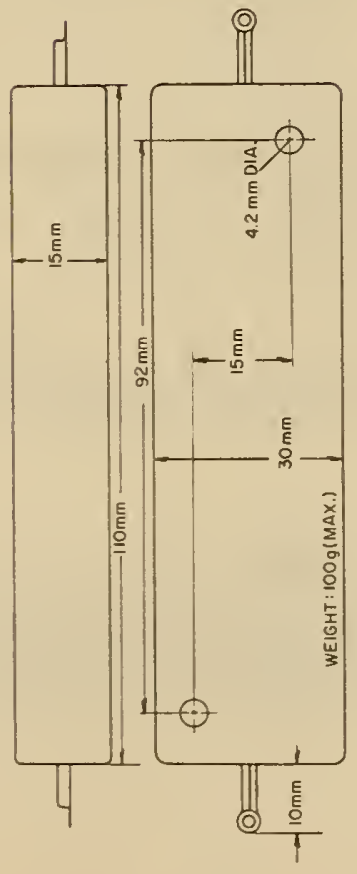

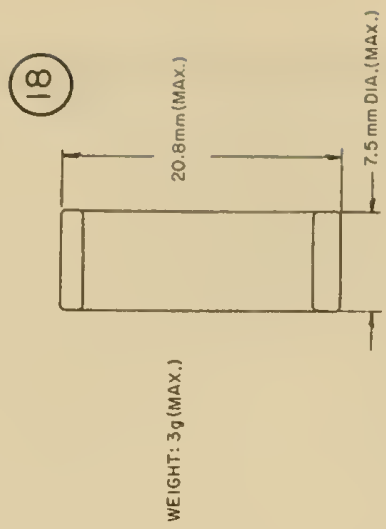

()

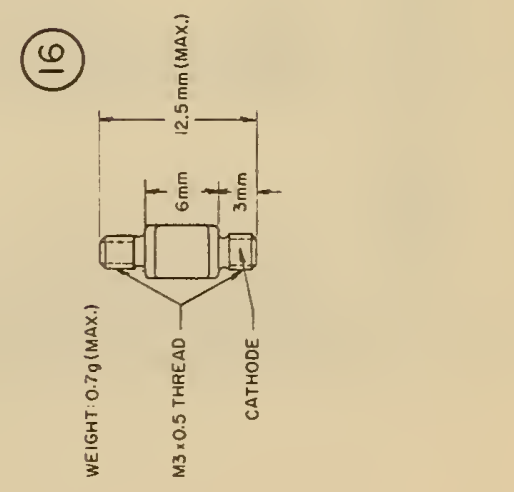




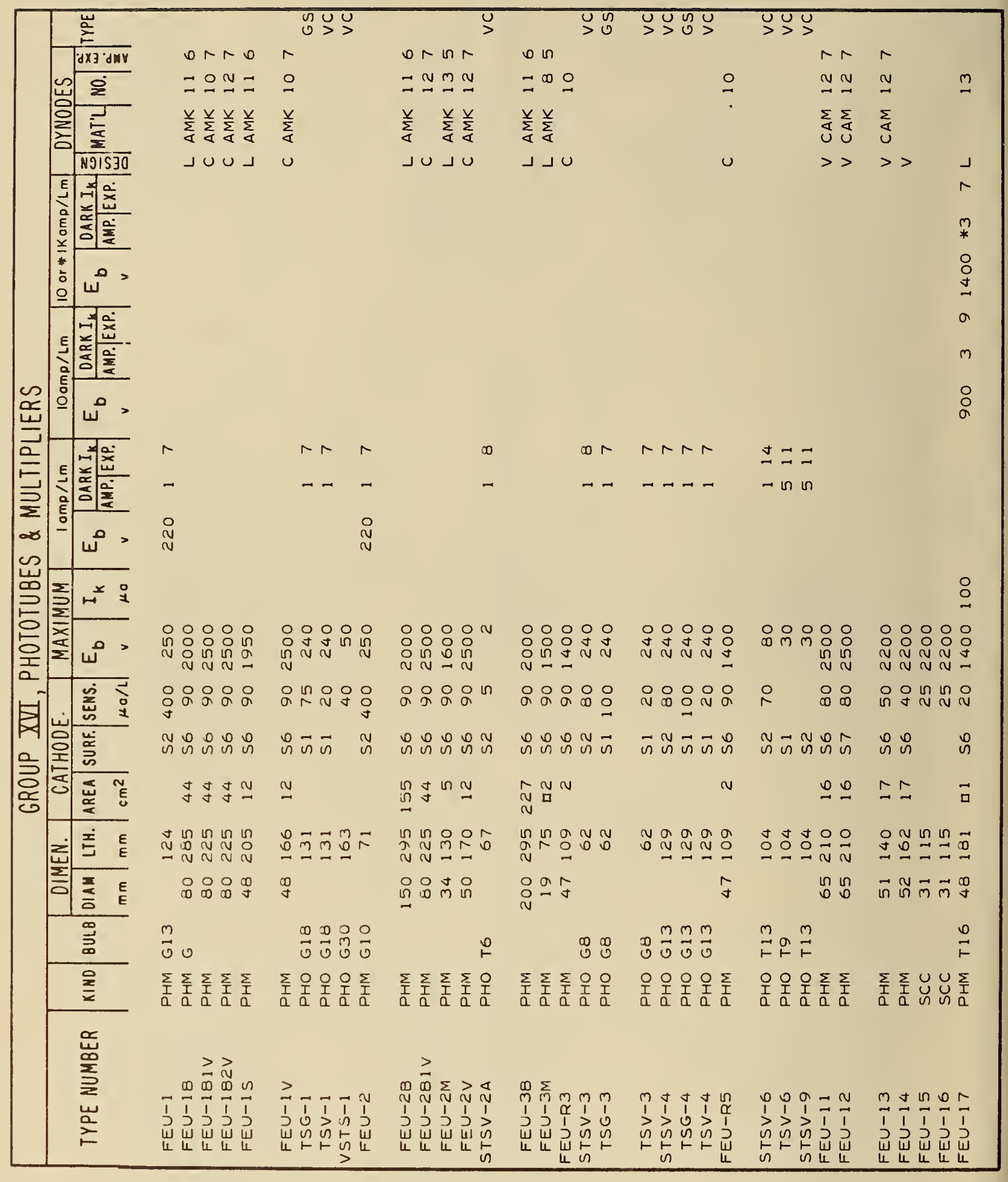




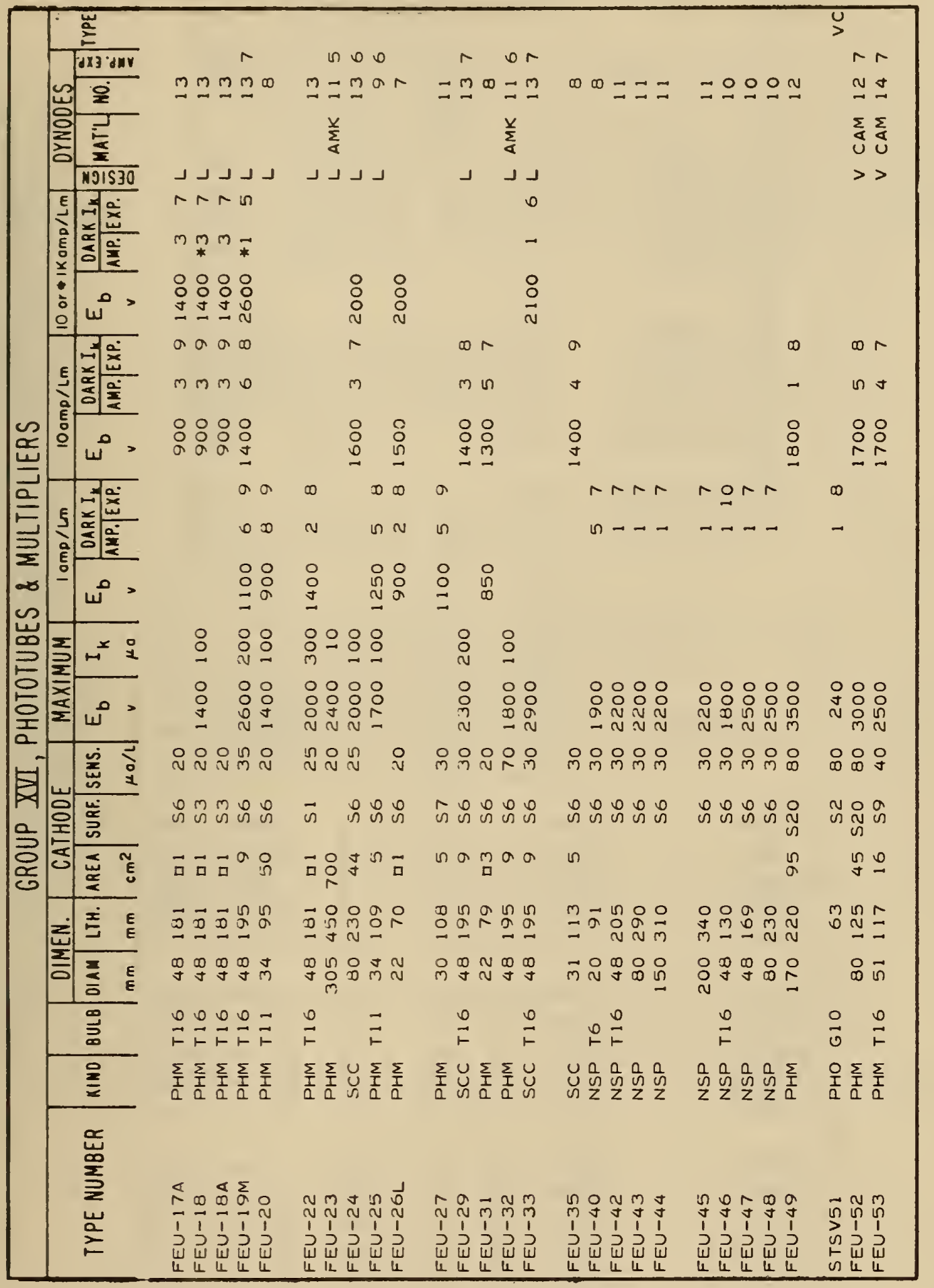




\begin{tabular}{|c|c|c|c|c|c|}
\hline \multicolumn{6}{|c|}{ GROUP XVII, FLASH TUBES } \\
\hline \multirow[b]{2}{*}{ TYPE NUMBER } & \multirow[b]{2}{*}{ KIND } & \multirow{2}{*}{$\mid$\begin{tabular}{|} 
BULB \\
SHAPE \\
AND \\
SIZE
\end{tabular}} & \multicolumn{2}{|c|}{ MAXIMUM } & \multirow{2}{*}{$\begin{array}{c}\text { TYP } \\
\text { TUBE } \\
\text { OROP } \\
v\end{array}$} \\
\hline & & & $\underset{v}{\text { VOLT. }}$ & $\begin{array}{c}\text { POWER } \\
\text { W }\end{array}$ & \\
\hline $1 S T-10$ & FLS & $u$ & $10 H$ & $10 \cdot 0$ & $18 n$ \\
\hline IF $K-20$ & FLS & T6 & 700 & $2 \cdot 0$ & $10 n$ \\
\hline IFK -50 & FLS & T6 & $10 \mathrm{H}$ & 5.0 & $14 n$ \\
\hline $\begin{array}{l}\text { IFK-120 } \\
\text { IFP-200 }\end{array}$ & FLS & UT & $\begin{array}{l}1 \mathrm{OH} \\
2 \mathrm{OH}\end{array}$ & $\begin{array}{l}12.0 \\
27.0\end{array}$ & $\begin{array}{l}180 \\
450\end{array}$ \\
\hline I F $8-300$ & FLS & $u$ & $15 \mathrm{H}$ & 40.0 & 240 \\
\hline IFK -500 & FLS & $H$ & $35 H$ & $30 \cdot 0$ & $40 n$ \\
\hline IFP-500 & FLS & ST 3 & $30 \mathrm{H}$ & 65.0 & $45 n$ \\
\hline IFP-1500 & FLS & T3 & $40 \mathrm{H}$ & HIO & $90 n$ \\
\hline IFK -2000 & FLS & $u$ & $20 \mathrm{H}$ & H3O & $25 n$ \\
\hline IFP-4000 & FLS & $T 3$ & $5 \mathrm{OH}$ & $\mathrm{H} 27$ & $1 \mathrm{~K}$ \\
\hline IFP-15000 & FLS & $T 4$ & $5 \mathrm{OH}$ & K12 & $1 K$ \\
\hline
\end{tabular}

\begin{tabular}{|c|c|c|c|c|c|c|c|}
\hline \multicolumn{8}{|c|}{ GROUP XVII, THERMOCOUPLE } \\
\hline \multirow[b]{2}{*}{ TYPE NUMBER } & \multirow[b]{2}{*}{ KINO } & \multicolumn{2}{|c|}{ OIMENSIONS } & \multicolumn{2}{|c|}{ TYPICAL } & \multirow{2}{*}{ 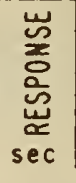 } & \multirow[b]{2}{*}{$\begin{array}{c}f_{\max } \\
m c\end{array}$} \\
\hline & & $\begin{array}{c}\text { DIAM } \\
\mathrm{mm}\end{array}$ & $\left|\begin{array}{c}\text { LENGTH } \\
\mathrm{mm}\end{array}\right|$ & $\begin{array}{l}I_{H} \\
\text { mo }\end{array}$ & $\begin{array}{c}\text { THERMO } \\
\text { ELEC } \\
\mathrm{mv}\end{array}$ & & \\
\hline$T V B-1$ & THM & 20 & 30 & 1 & $\square 3$ & 40 & 200 \\
\hline$T V-2$ & THM & 13 & 23 & $10 n$ & $3 n$ & 35 & 5 \\
\hline TVB-2 & THM & 20 & $3 n$ & 3 & 5 & $4 n$ & 200 \\
\hline TVB-3 & THM & 20 & 30 & 5 & in & 40 & 200 \\
\hline$T V-4$ & THM & 13 & 23 & 50 & 30 & 35 & 5 \\
\hline TVB-4 & THM & 20 & 30 & 10 & 12 & 40 & 200 \\
\hline$T V-5$ & THM & 13 & 23 & 75 & 30 & 35 & 5 \\
\hline$T V B-5$ & THM & 20 & 30 & 30 & 12 & 40 & 200 \\
\hline$T V B-6$ & THM & 20 & 30 & 30 & 12 & 40 & 200 \\
\hline$T V B-7$ & THM & 20 & 30 & 100 & 12 & 40 & 200 \\
\hline TVB-8 & THM & 20 & 30 & 300 & 12 & 40 & 200 \\
\hline TVB-9 & THM & 20 & 30 & 500 & 12 & 40 & 200 \\
\hline$T V-14$ & THM & 13 & 23 & 250 & $3 n$ & 15 & $\because 5$ \\
\hline$T V-15$ & THM & 15 & 20 & 500 & 30 & 35 & 5 \\
\hline$T V-16$ & THM & 15 & 20 & 1000 & $3 n$ & 35 & 5 \\
\hline
\end{tabular}




\begin{tabular}{|c|c|c|c|c|c|c|c|c|c|c|c|c|c|}
\hline \multirow[b]{2}{*}{ TYPE NUMBER } & \multirow[b]{2}{*}{ KINO } & \multirow[b]{2}{*}{ USE } & \multicolumn{2}{|c|}{ OIMEN } & \multirow[b]{2}{*}{ SHAPE } & \multicolumn{3}{|c|}{ RESISTANCE } & \multicolumn{2}{|c|}{ TEMP. } & \multicolumn{2}{|c|}{ POWER } & \multirow[b]{2}{*}{$\begin{array}{l}\text { SENS } \\
\frac{\Omega}{\min w}\end{array}$} \\
\hline & & & $\begin{array}{c}\text { OIAK } \\
\mathrm{mm}\end{array}$ & $\begin{array}{l}\text { LTH } \\
\mathrm{mm}\end{array}$ & & $\begin{array}{c}M \| N \\
\Omega\end{array}$ & $\begin{array}{c}\text { MAX } \\
\Omega\end{array}$ & $\begin{array}{l}\text { T. C. } \\
\%\end{array}$ & 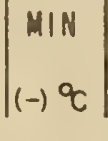 & $\left|\begin{array}{c}\max \\
(t) \\
q\end{array}\right|$ & $\begin{array}{l}M I N \\
m w\end{array}$ & $\left|\begin{array}{l}\text { MaX } \\
\text { mw }\end{array}\right|$ & \\
\hline$K M T-1$ & TMS & MEA & 13 & $\square 4$ & CYL & $20 K$ & $1 M$ & $5 \cdot 1$ & 20 & 180 & & $8 H$ & \\
\hline MMT-1 & TMS & MEA & 13 & $\square 4$ & CYL & 1 & 200 & $2 \cdot 9$ & 70 & 120 & & $4 \mathrm{H}$ & \\
\hline$K M T-4$ & TMS & MEA & 24 & 7 & CYL & $20 K$ & $I M$ & $5 \cdot 1$ & 20 & 180 & & $8 \mathrm{H}$ & \\
\hline$M M T-4$ & TMS & MEA & 24 & 7 & CYL & 1 & 200 & $2 \cdot 9$ & 70 & 120 & & & \\
\hline$M M T-6$ & TMS & & & & & 10 & 1000 & $2 \cdot 9$ & 70 & 120 & & 50 & \\
\hline$K M T-8$ & TMS & & & & & 100 & $10 K$ & $4 \cdot 6$ & 40 & 60 & & & \\
\hline$M M T-8$ & TMS & COM & 22 & 23 & DSC & 1 & 1000 & $2 \cdot 9$ & 40 & 60 & & 10 & \\
\hline T80 & TMS & POW & 8 & 3 & CYL & 150 & & & & & 10 & 15 & 20 \\
\hline T8E & TMS & POW & 8 & 3 & CYL & 150 & & & & & 7 & 10 & 30 \\
\hline T $8 M$ & TMS & POW & 8 & 3 & CYL & 200 & & & & & 9 & 11 & 66 \\
\hline T8R & TMS & POW & 8 & 3 & $C Y L$ & 125 & & & & & 7 & 12 & 10 \\
\hline T851 & $\begin{array}{l}\text { TMS } \\
\text { TMS }\end{array}$ & $\begin{array}{l}\text { POW } \\
\text { POW }\end{array}$ & $\begin{array}{l}8 \\
8\end{array}$ & $\begin{array}{l}3 \\
3\end{array}$ & $\begin{array}{l}\text { CYL } \\
\text { CYL }\end{array}$ & 120 & & & & & $9 \cdot 5$ & $\begin{array}{l}24 \\
24\end{array}$ & 10 \\
\hline $\begin{array}{l}\text { T8S1M } \\
\text { T852 }\end{array}$ & TMS & POW & 8 & $\begin{array}{l}3 \\
3\end{array}$ & CYL & $\begin{array}{l}1<0 \\
150\end{array}$ & & & & & 8 & 19 & 12 \\
\hline T852M & $T M S$ & POW & 8 & 3 & CYL & 150 & & & & & 8 & 19 & 12 \\
\hline T8S3 & TMS & POW & 8 & 3 & CYL & 150 & & & & & 7 & 23 & 10 \\
\hline T8S3M & $T M S$ & POW & 8 & 3 & CYL & 150 & & & & & 7 & 23 & 10 \\
\hline$M M T-9$ & TMS & COM & $\square 3$ & 19 & DSC & 10 & 5000 & $2 \cdot 9$ & 60 & 120 & & 10 & \\
\hline T9 & TMS & POW & 8 & 3 & CYL & 125 & & & & & 7 & 19 & 10 \\
\hline KMTIO & TMS & CON & 30 & 6 & $C Y L$ & $100 \mathrm{~K}$ & $3 M$ & $5 \cdot 1$ & 0 & 120 & & $2 \mathrm{H}$ & \\
\hline $\begin{array}{l}K M T-11 \\
K M T-12\end{array}$ & $\begin{array}{l}T M S \\
T M S\end{array}$ & CON & -44 & ㅁ 1 & CYL & $\begin{array}{r}100 K \\
100\end{array}$ & $\begin{array}{r}3 M \\
10 K\end{array}$ & $\begin{array}{l}5 \cdot 1 \\
4 \cdot 6\end{array}$ & 40 & $\begin{array}{l}120 \\
120\end{array}$ & & $2 \mathrm{H}$ & \\
\hline MMT -12 & TMS & & & & & 5 & $5 K$ & $2 \cdot 9$ & 40 & 120 & & 3 & \\
\hline
\end{tabular}




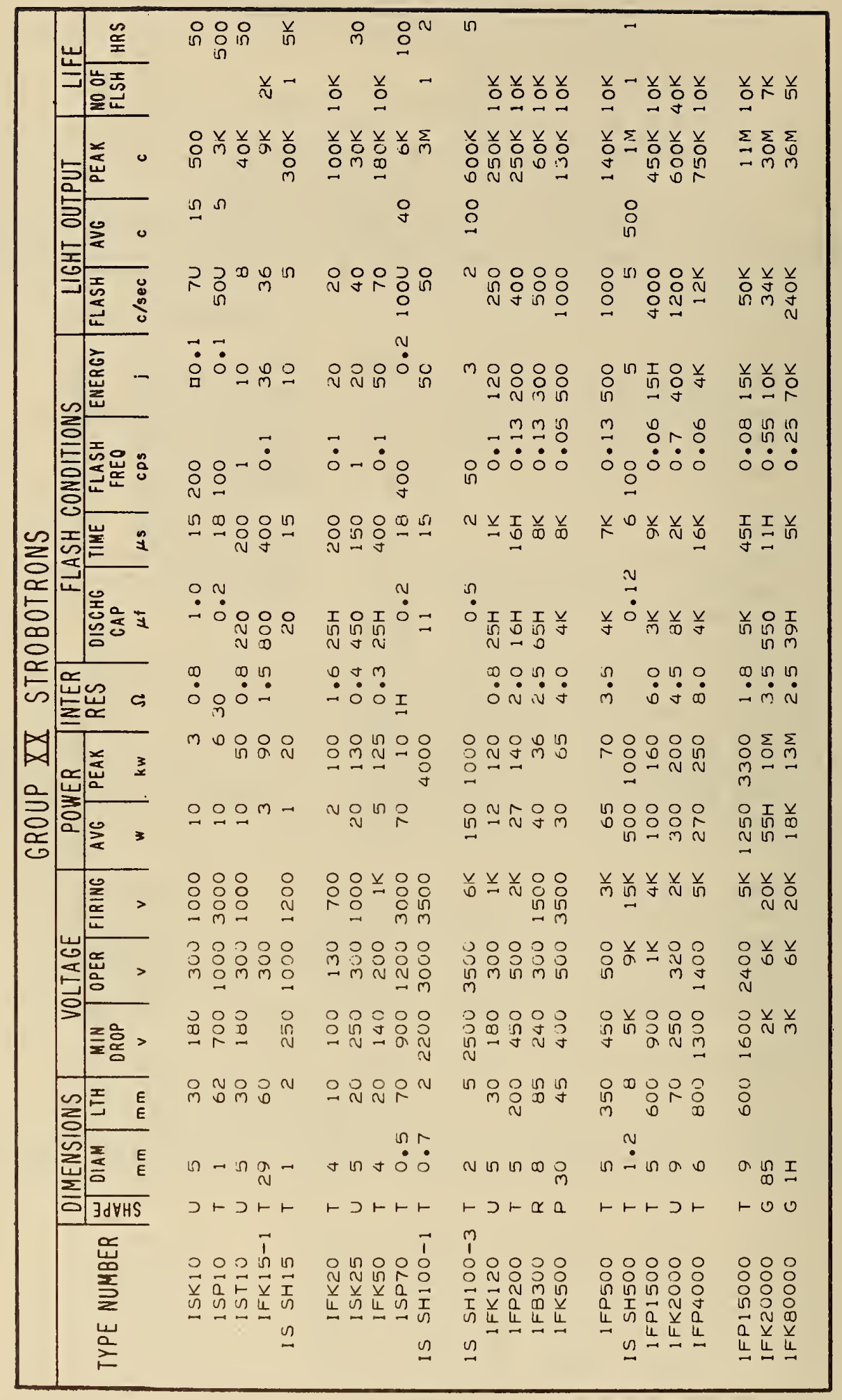




\begin{tabular}{|c|c|c|c|c|c|c|c|c|c|c|c|c|c|c|c|c|}
\hline \multirow{3}{*}{ TYPE NUMBER } & \multirow{3}{*}{ KINO } & \multirow{3}{*}{ 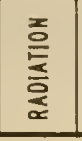 } & \multirow{3}{*}{$\begin{array}{l}\text { 옹 } \\
\text { 퐁 } \\
\text { 岁 } \\
\end{array}$} & \multirow{3}{*}{ 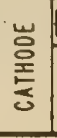 } & \multicolumn{2}{|c|}{ DIMENSIONS } & \multicolumn{2}{|c|}{ PLATEAU } & \multicolumn{3}{|c|}{ MAXIMUM } & \multicolumn{2}{|c|}{ TEMP } & \multirow{3}{*}{$\begin{array}{l}\text { CAP } \\
\text { pf }\end{array}$} & \multirow{3}{*}{$\begin{array}{c}M I N \\
R_{i} \\
\text { meg } \\
\Omega\end{array}$} & \multirow{3}{*}{ FIG } \\
\hline & & & & & \multirow{2}{*}{$\begin{array}{c}\text { OIAM } \\
\mathrm{mm}\end{array}$} & \multirow{2}{*}{$\begin{array}{c}\text { LENCTH } \\
\mathrm{mm}\end{array}$} & \multirow{2}{*}{$\begin{array}{c}M I N \\
v \\
\end{array}$} & \multirow{2}{*}{$\begin{array}{c}\text { MAX } \\
v \\
\end{array}$} & \multirow{2}{*}{$\begin{array}{l}\text { RATE } \\
10^{3} / \mathrm{m} \text { in }\end{array} \mid$} & \multicolumn{2}{|c|}{ PLATEAU } & \multirow{2}{*}{$\begin{array}{r}M I N \\
-q\end{array}$} & MAX & & & \\
\hline & & & & & & & & & & $\underset{v}{10 T H}$ & $\begin{array}{l}\text { SCOPE } \\
\% \mathrm{~V}\end{array}$ & & $+{ }^{\circ} \mathrm{C}$ & & & \\
\hline$S 1-18 G$ & $\mathrm{cou}$ & $B A G$ & SQ & $\mathrm{NI}$ & 60 & 15 & 375. & 4,10 & & & & 40 & 50 & 5 & ㅁ⒈ & 11 \\
\hline$S I-1 G$ & $\mathrm{cou}$ & BET & $\mathrm{SO}$ & FE & 94 & 16 & 280 & 320 & 60 & 80 & $1 \cdot 25$ & 40 & 50 & 10 & 5 & 8 \\
\hline STS-2 & cou & BET & So & FE & 180 & 24 & 285 & 335 & 40 & 80 & 1.25 & 40 & 50 & 10 & 5 & 9 \\
\hline$S I-2 B$ & $\mathrm{COU}$ & BET & so & $S N$ & 90 & 70 & 1350 & 1750 & 8 & 150 & 0.5 & 30 & 50 & 10 & 7 & 13 \\
\hline$S 1-2 B G$ & $\mathrm{cou}$ & $B A G$ & so & NI & 60 & 15 & 375 & 410 & & & & 40 & 50 & 5 & 3 & 12 \\
\hline STS-3 & $\mathrm{cou}$ & BET & so & FE & 265 & 23 & 285 & 335 & 30 & 80 & $1 \cdot 25$ & 40 & 50 & 10 & 5 & 8 \\
\hline$S I-3 B$ & $\mathrm{cov}$ & BET & so & $\mathrm{Cu}$ & 90 & 40 & 1650 & & 10 & 150 & 0.3 & 20 & 40 & 10 & 7 & 6 \\
\hline GS-4 & $\mathrm{cou}$ & GAM & $S Q$ & GR & 180 & 23 & 1100 & 1300 & & 200 & 1.0 & & & 25 & 8 & 3 \\
\hline MS -4 & $\mathrm{cou}$ & GAM & so & $\mathrm{Cu}$ & 180 & 23 & 720 & 780 & 25 & 200 & 1.0 & 40 & 50 & 25 & 8 & 4 \\
\hline MSTR-4 & $\mathrm{cou}$ & BET & SQ & $\mathrm{CU}$ & 180 & 40 & 1200 & 1350 & 25 & 200 & 0.5 & 5 & 35 & 25 & 8 & 7 \\
\hline$S 1-4 G$ & $\mathrm{cou}$ & GAM & SQ & $w$ & 367 & 33 & 720 & 800 & 25 & 200 & 1.0 & 40 & 150 & 25 & 8 & 1 \\
\hline VS-4 & cou & GAM & SQ & $w$ & 180 & 23 & 720 & 800 & 25 & 200 & 0.75 & 40 & 50 & 25 & 8 & 1 \\
\hline STS -5 & $\mathrm{cou}$ & $B E T$ & so & FE & 113 & 12 & 280 & 330 & 100 & 80 & 1.25 & 40 & 50 & 10 & 5 & 9 \\
\hline GS-6 & $\mathrm{cou}$ & GAM & so & GR & 266 & 23 & 1100 & 1300 & & 200 & 1.0 & & & 25 & 8 & 3 \\
\hline$M S-6$ & $\mathrm{cou}$ & GAM & so & $\mathrm{Cu}$ & 266 & 23 & 720 & 780 & 25 & 200 & 1.0 & 40 & 50 & 25 & 8 & 4 \\
\hline STS-6 & cou & BET & SO & FE & 200 & 22 & 285 & 335 & 60 & 80 & 1.25 & 40 & 50 & 10 & 5 & 10 \\
\hline VS-6 & $\mathrm{cou}$ & GAM & so & $w$ & 266 & 23 & 720 & 800 & 25 & 200 & 0.75 & 40 & 50 & 25 & 8 & 1 \\
\hline GS -7 & $\mathrm{cou}$ & GAM & so & GR & 145 & 16 & 1100 & 1300 & & 150 & 1.0 & & & 25 & 30 & 3 \\
\hline$M S-7$ & $\mathrm{COU}$ & GAM & so & $\mathrm{CU}$ & 145 & 16 & 720 & 780 & 25 & 100 & 1.5 & 25 & 50 & 25 & 30 & 4 \\
\hline GS -8 & $\mathrm{cou}$ & GAM & so & GR & 185 & 16 & 1100 & 1300 & & 150 & 1.0 & & & 25 & 30 & 3 \\
\hline$M S-8$ & cou & GAM & so & $\mathrm{CU}$ & 185 & 16 & 720 & 780 & 25 & 100 & $1 \cdot 5$ & 25 & 50 & 25 & 30 & 4 \\
\hline STS-8 & $\mathrm{cou}$ & BET & SQ & FE & 220 & 23 & 285 & 335 & 40 & 80 & $1 \cdot 25$ & 40 & 50 & 10 & 5 & 8 \\
\hline VS-8 & cou & GAM & so & $w$ & 185 & 16 & 720 & 800 & 25 & 150 & $1 \cdot 0$ & 40 & 50 & 25 & 30 & 1 \\
\hline GS-9 & $\mathrm{cou}$ & GAM & SQ & $G R$ & 367 & 33 & 1100 & 1300 & & 250 & 1.0 & & & 25 & 8 & 3 \\
\hline$M S-9$ & $\mathrm{cou}$ & GAM & SQ & $\mathrm{CU}$ & 367 & 33 & 720 & 780 & 25 & 250 & $1 \cdot 0$ & 40 & 50 & 25 & 8 & 4 \\
\hline VS -9 & $\mathrm{cou}$ & GAM & so & $w$ & 367 & 33 & 720 & 800 & 25 & 250 & 0.75 & 40 & 50 & 25 & 8 & 1 \\
\hline$G S-10$ & cou & GAM & so & GR & 225 & 16 & 1100 & I 300 & & 150 & $1 \cdot 0$ & & & 25 & 30 & 3 \\
\hline$G S-11$ & $\mathrm{cou}$ & GAM & so & GR & 185 & 33 & 1100 & 1300 & & 200 & 1.0 & & & 25 & 8 & 3 \\
\hline$M S-11$ & cou & GAM & so & $\mathrm{CU}$ & 185 & 33 & 720 & 780 & 25 & 200 & 1.0 & 40 & so & 25 & 8 & 4 \\
\hline VS -11 & cou & GAM & so & $w$ & 185 & 33 & 720 & 800 & 25 & 200 & 0.75 & 40 & 50 & 25 & 8 & 1 \\
\hline$G S-12$ & cou & GAM & so & GR & 145 & 16 & 1100 & 1300 & & 150 & $1 \cdot 0$ & & & 25 & 30 & 3 \\
\hline$M S-12$ & cou & GAM & SQ & $\mathrm{CU}$ & 145 & 16 & 720 & 780 & 25 & 100 & 1.5 & 25 & 50 & 25 & 30 & 4 \\
\hline$M S-13$ & cou & GAM & SQ & $\mathrm{CU}$ & 100 & 23 & 720 & 780 & 25 & 200 & 1.5 & 40 & 50 & 25 & 8 & 5 \\
\hline$V S-13$ & cou & GAM & so & $w$ & 100 & 23 & 720 & 800 & 25 & 150 & 1.0 & 40 & 50 & 25 & 8 & 2 \\
\hline$M S-14$ & cou & GAM & so & $\mathrm{CU}$ & 160 & 23 & 720 & 780 & 25 & 200 & 1.0 & 40 & 50 & 25 & 8 & 5 \\
\hline VS- 14 & $\mathrm{cou}$ & GAM & so & $w$ & 160 & 23 & 720 & 800 & 25 & 200 & 0.75 & 40 & 50 & 25 & 8 & 2 \\
\hline$M S-16$ & cou & GAM & SQ & $\mathrm{Cu}$ & 250 & 23 & 720 & 780 & 25 & 200 & $1 \cdot 0$ & 40 & 50 & 25 & 8 & 5 \\
\hline VS-16 & $\mathrm{cou}$ & GAM & so & $w$ & 250 & 23 & 720 & 800 & 25 & 200 & 0.75 & 40 & 50 & 25 & 8 & 2 \\
\hline$M S T-11$ & $\mathrm{cou}$ & $B E T$ & SQ & CU & 100 & 40 & 1600 & & 10 & 150 & 0.5 & 30 & 50 & 10 & 7 & 6 \\
\hline GS-30 & cou & GAM & so & GR & 662 & 33 & 1100 & 1300 & & 150 & 1.0 & & & 25 & 8 & 3 \\
\hline$G S-60$ & cou & GAM & SO & $G R$ & 667 & 63 & 1100 & 1300 & & 150 & $1 \cdot 0$ & & & 25 & 8 & 3 \\
\hline
\end{tabular}




\begin{tabular}{|c|c|c|c|c|c|c|c|c|c|c|c|c|c|c|}
\hline & & & GRO & UP & XI & III 0 & ISCH & ARGE & 01 & ODES & & & & \\
\hline & & VEN & & & ATH & FIR & ING & & PUI & SE & $M \mid N$ & MAX & AMB. & TEMP \\
\hline TYPE NUMBER & $\begin{array}{l}\text { LTH } \\
\mathrm{mm}\end{array}$ & $\begin{array}{l}\text { DIAM } \\
\mathrm{mm}\end{array}$ & GAS & 嵅 & KINO & $\begin{array}{l}\text { MIN } \\
v\end{array}$ & $\begin{array}{c}\operatorname{MAX} \\
v\end{array}$ & \begin{tabular}{|l|} 
I-amp \\
J-joule
\end{tabular} & \begin{tabular}{|l|} 
TIME \\
sec
\end{tabular} & $\begin{array}{l}\text { OPERATING } \\
\text { FREQUENCY } \\
\text { CDS }\end{array}$ & $\mid \begin{array}{c}\text { INTER } \\
\text { RES } \\
\text { mea } \Omega\end{array}$ & $\mid \begin{array}{l}\text { CAP } \\
\text { of }\end{array}$ & \begin{tabular}{|l|}
$M I N$ \\
$(-)^{\circ} \mathrm{C}$
\end{tabular} & $\begin{array}{l}\operatorname{Max} \\
(+)^{\circ} \mathrm{C}\end{array}$ \\
\hline$R B=1$ & 52 & 19 & & c & $B A$ & 150 & 190 & & & & 400 & & & \\
\hline$R-2$ & 17 & $16 \cdot 5$ & & c & & 1300 & $2 k$ & & & 600 & 20 & & 50 & 80 \\
\hline$R B-2$ & 25 & 19 & & c & $B A$ & & 220 & 50 & $15 U$ & 50 & 100 & व1 & 60 & 70 \\
\hline$R-3$ & 70 & 21.5 & & c & $B A O$ & & 600 & 140 & 120 & 300 & 100 & 1 & 60 & 70 \\
\hline$R B=3$ & 41 & 22 & & $c$ & $B A$ & 220 & 235 & 30 & IHU & 7 & 100 & & 60 & 70 \\
\hline$R-4$ & & & & c & $B A O$ & & 75 & & & & & & & \\
\hline$R-5$ & 41 & 22 & & c & $B A O$ & 160 & 250 & & & & 100 & & & \\
\hline$R B-5$ & 60 & 16 & & c & $B A$ & 340 & 460 & $10 \mathrm{~J}$ & & 1 & 200 & & 60 & 70 \\
\hline$R B-5 A$ & 60 & 16 & & c & $B A$ & 370 & 510 & alJ & & 8 & & & 60 & 50 \\
\hline R6 & 110 & 55 & & & & $8 \cap 0$ & & & & $200 \mathrm{M}$ & 100 & & & \\
\hline$R-7$ & 45 & 18 & $H K$ & $c$ & $B A O$ & 270 & 330 & & & 2 & 20 & 10 & 60 & 100 \\
\hline$R-8$ & 50 & 20 & HK & $c$ & $B A O$ & 450 & 550 & & & 2 & 20 & 10 & 60 & 100 \\
\hline$R-9$ & 55 & 20 & $H K$ & c & $B A O$ & 900 & 1100 & & & 2 & 20 & 10 & 60 & 100 \\
\hline$R-10$ & 55 & 20 & HK & $c$ & $B A O$ & 1375 & 1725 & & & 2 & 20 & 40 & 60 & 100 \\
\hline$R-11$ & 132 & 35 & & $c$ & NI & 2250 & 2750 & $2 \mathrm{HU}$ & & & & & & \\
\hline $\begin{array}{l}R-12 \\
R-54\end{array}$ & 30 & 12 & $A R$ & c & K & $\begin{array}{r}145 \\
7200\end{array}$ & $\begin{array}{r}175 \\
9800\end{array}$ & 20 & IU & & 1000 & & & \\
\hline$R B-90$ & 62 & 17.5 & NA & $c$ & $B A$ & 80 & 100 & $30 M$ & 2 & 0.005 & 100 & 100 & 60 & 70 \\
\hline$S K-127$ & 37 & 20 & NA & & MG & & 72 & 1 & 20 & 1 & & & & \\
\hline$S K-220$ & 37 & 20 & HE & & & & 140 & 口1 & 20 & $i$ & & & & \\
\hline$R B-280$ & 210 & 95 & $A R$ & $c$ & $B A$ & 250 & 310 & 30 & 10 & 0.002 & 40 & 20 & 60 & 70 \\
\hline$R-350$ & 62 & 20 & $A R$ & $c$ & $B A$ & 310 & 390 & 3 & 2 & 0.0 & $5 K$ & 10 & 50 & 50 \\
\hline$R B-350$ & 210 & 95 & $A R$ & $c$ & $B A$ & 310 & 390 & 30 & 10 & & 40 & 20 & 60 & 70 \\
\hline$R B-4.30$ & 210 & 95 & $A R$ & $c$ & $B A$ & 390 & 470 & 30 & 10 & & 40 & 20 & 60 & 70 \\
\hline$R-450$ & 62 & 20 & $A R$ & $c$ & $B A$ & 440 & 480 & 3 & 2 & 0.002 & $5 K$ & 10 & 50 & 50 \\
\hline
\end{tabular}

\begin{tabular}{|c|c|c|c|c|c|c|c|c|c|c|c|c|}
\hline \multicolumn{13}{|c|}{ GROUP XXTI DECATRONS } \\
\hline \multirow{3}{*}{ TYPE NUMBER } & \multirow{3}{*}{ KINO } & \multicolumn{6}{|c|}{ VOLTAGES } & \multirow{3}{*}{$\begin{array}{c}\text { TYP } \\
I_{b} \\
\text { mo }\end{array}$} & \multicolumn{2}{|c|}{ PULSE } & \multicolumn{2}{|c|}{ DIMEN } \\
\hline & & \multicolumn{4}{|c|}{ MAXIMUM } & \multicolumn{2}{|c|}{ TYPICAL } & & \multirow{2}{*}{\multicolumn{2}{|c|}{\begin{tabular}{|c|c|} 
TYP & MAX \\
TIME & RATE \\
$\mu \mathrm{S}$ & $\mathrm{KCS}$ \\
\end{tabular}}} & \multicolumn{2}{|c|}{ LTH } \\
\hline & & $E_{v}$ & FIRING & $\underset{v}{B \mid A S}$ & $\underset{v}{\operatorname{ORP}}$ & OPER & $K_{1} K_{2}$ & & & & $\mathrm{~mm}_{\mathrm{m}}$ & $\mathrm{mm}$ \\
\hline$O G-1$ & DEC & $45 n$ & 300 & 150 & 15 & 150 & 50 & $1 \cdot 3$ & 40 & 8 & 77 & 34 \\
\hline$O G-2$ & DEC & 450 & 300 & 150 & 15 & 150 & 50 & 1.3 & 60 & 3 & 77 & $\begin{array}{ll}7 & 34\end{array}$ \\
\hline$O G-3$ & DEC & 460 & 420 & 120 & 15 & & & 0.7 & 18 & 20 & 83 & \\
\hline$O G-5$ & DEC & 400 & 350 & 120 & 20 & 175 & 60 & 1.3 & 35 & 10 & 74 & $4 \quad 34$ \\
\hline
\end{tabular}

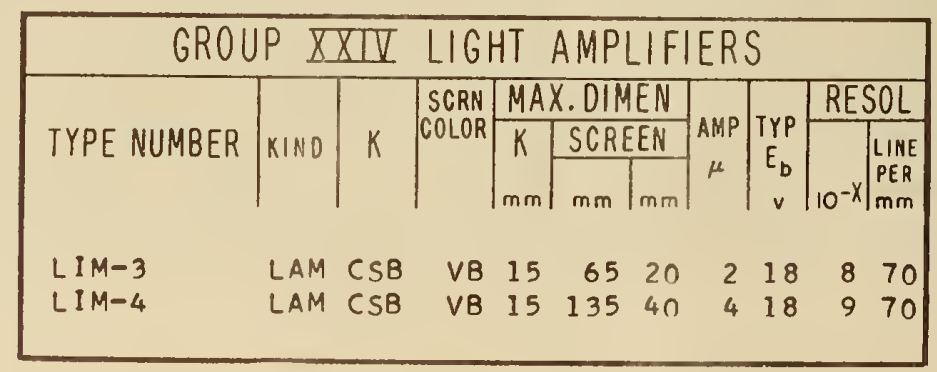




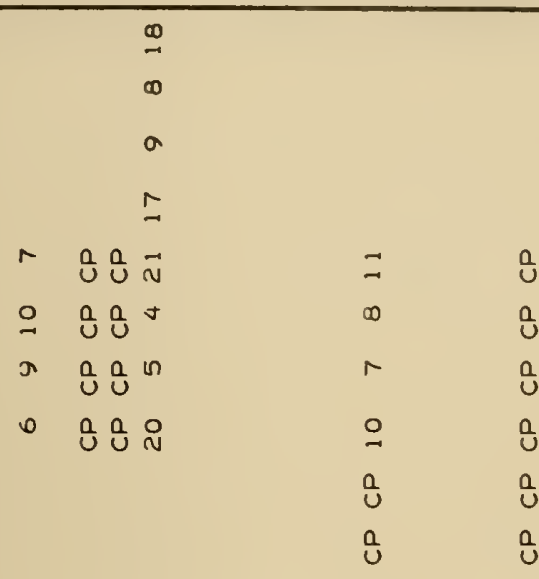

$a$
$m a$
0

$\cong \quad$

n

$\pm$

ต

ง $ง$

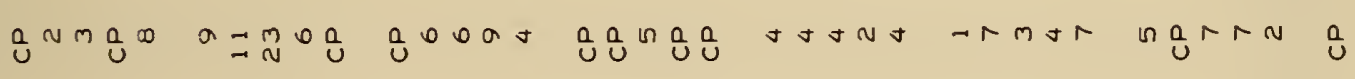

Dorm 00 ป

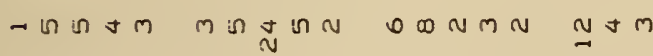

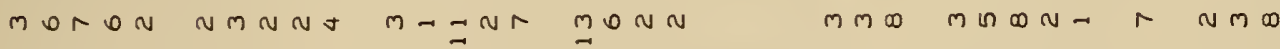
tror

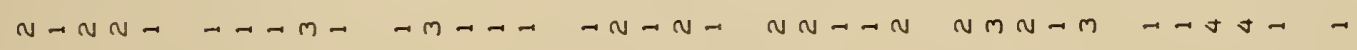

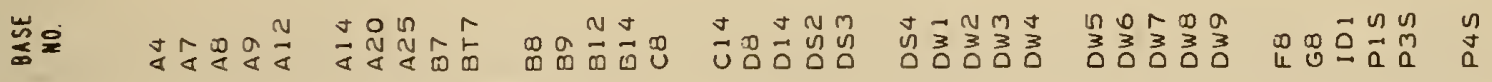




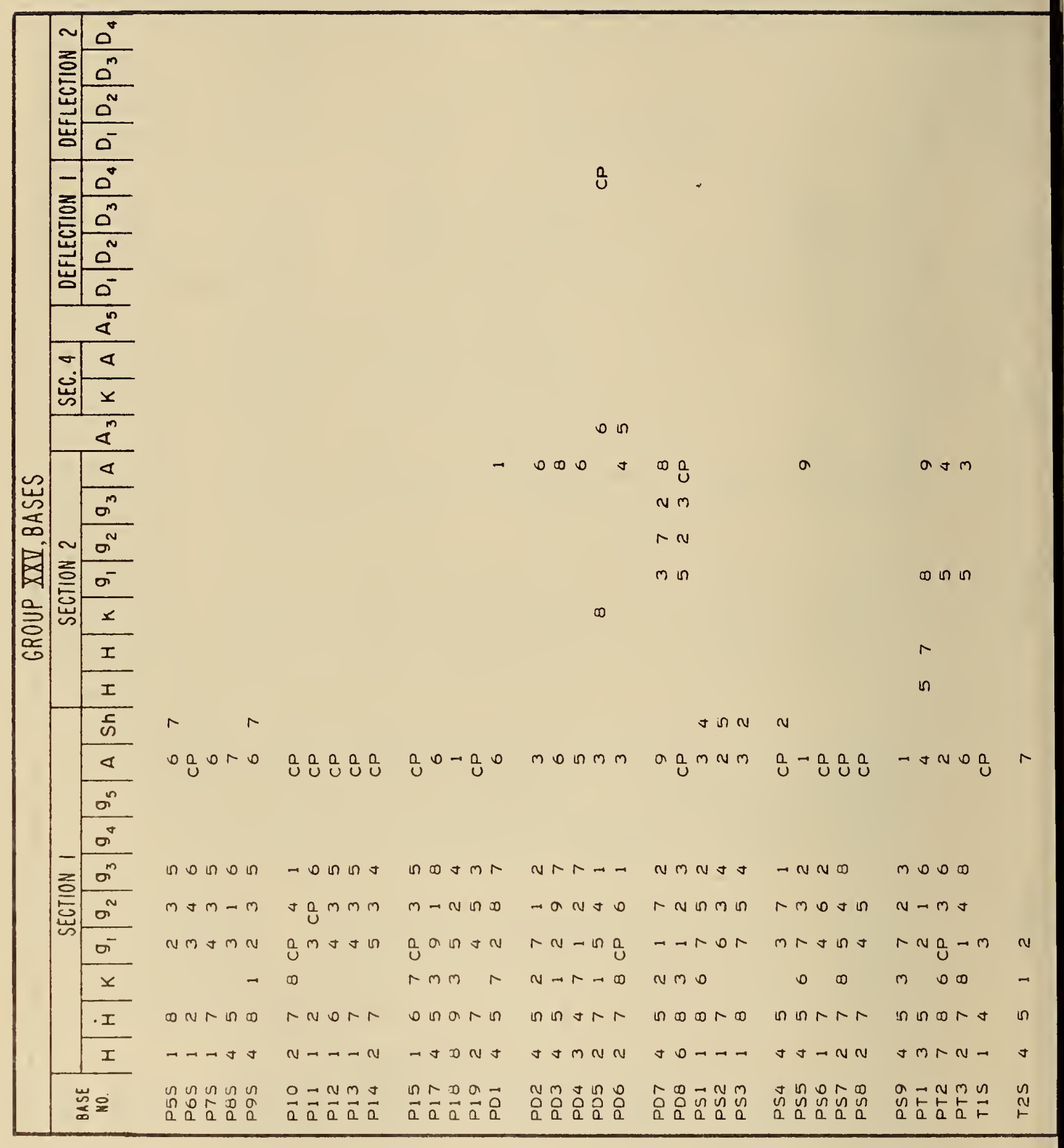




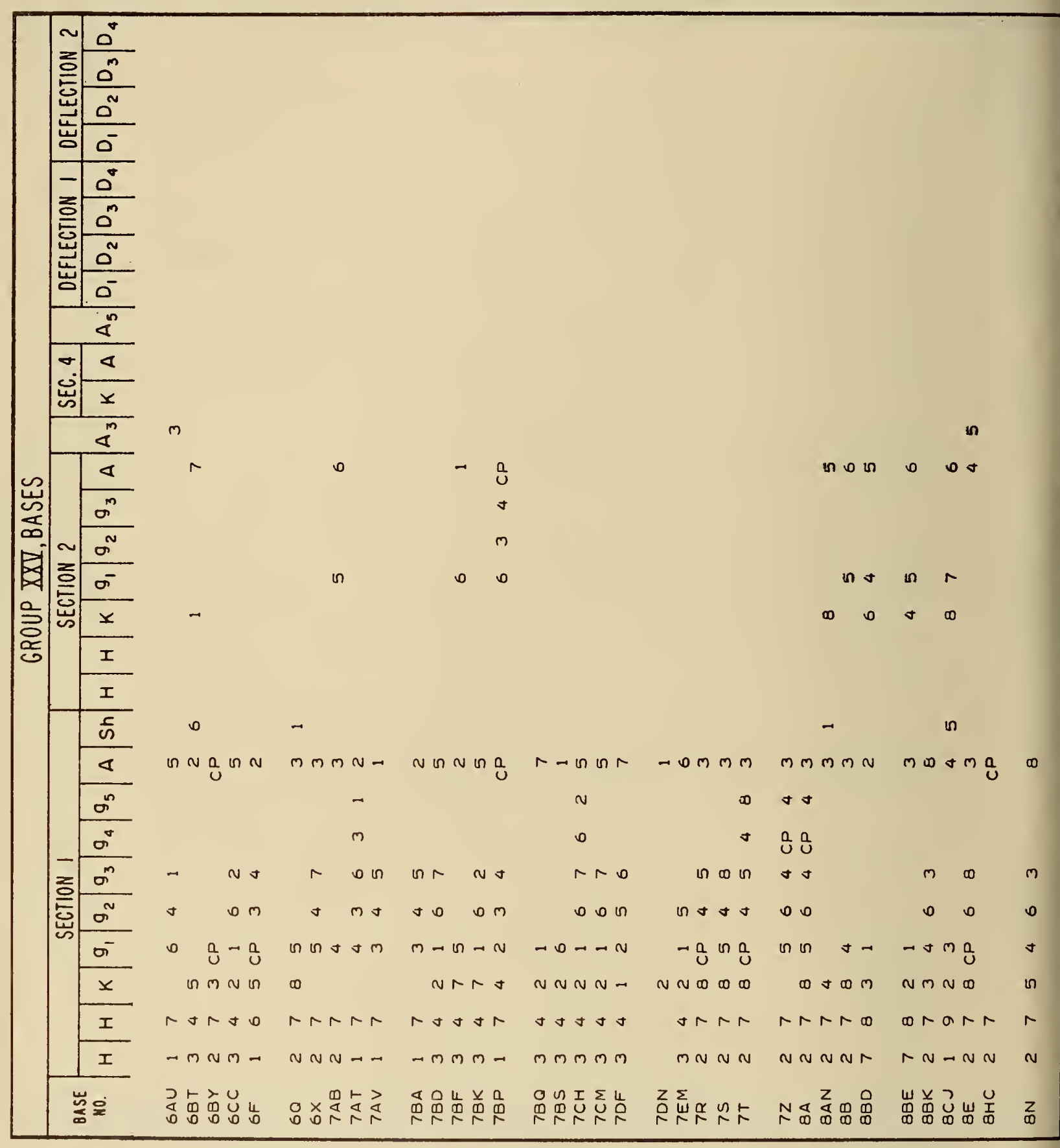




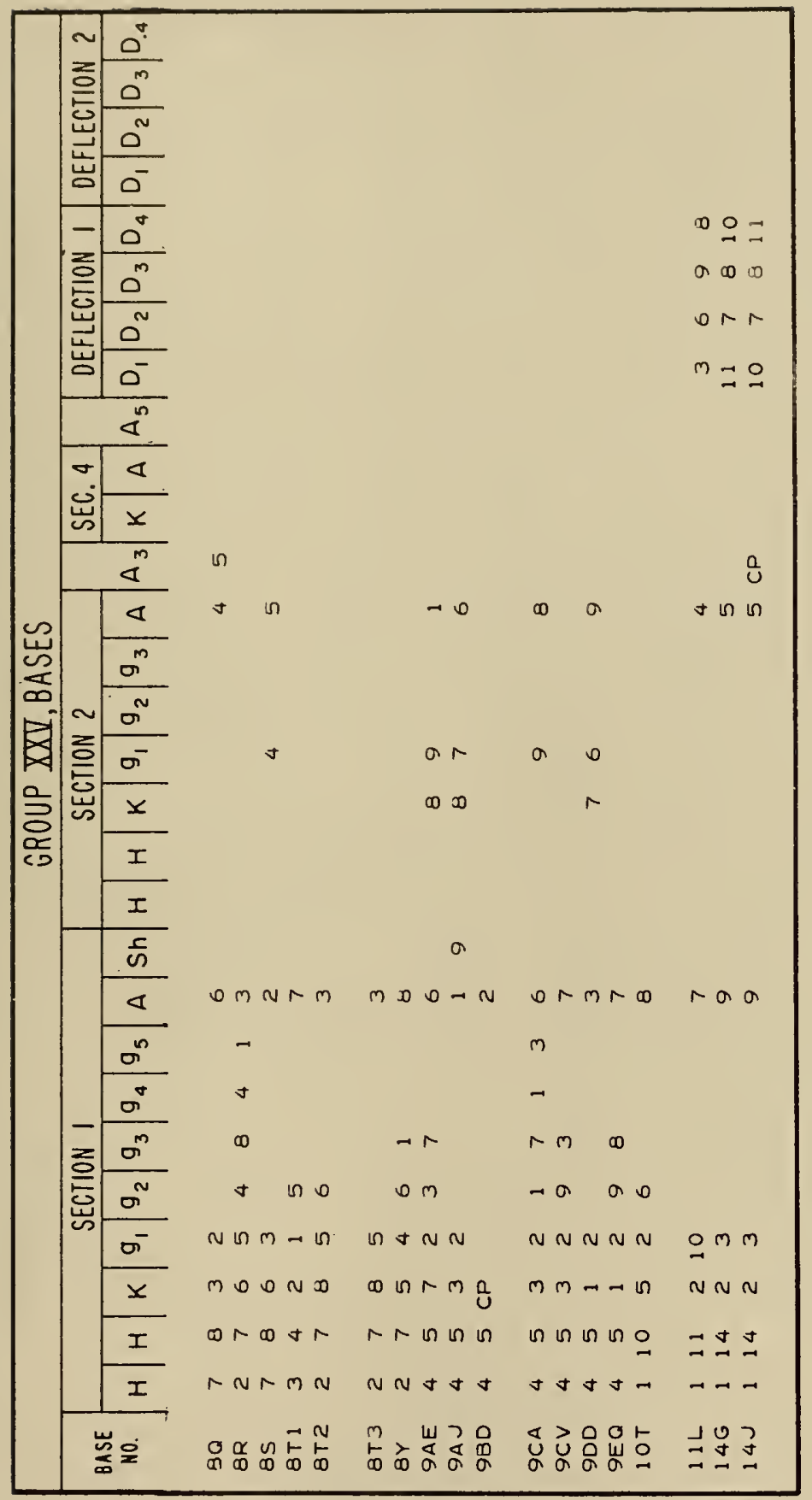



I.S. DEPIRTMENT OF COMNERCE

Luther II. Hodges, Secretary

NATIONAI, BUREAUI OF STANDARDS

A. I. Astin, Direcsor

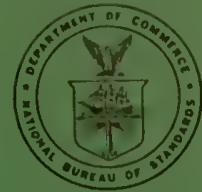

\section{THE NATIONAL BUREAU OF STANDARDS}

The scope of activities of the National Bureau of Standards at its major laboratories in Washington, D.C., and Boulder, Colorado, is suggested in the following listing of the divisions and sections engaged in technical work. In general, each section carries cut specialized research, development, and engineering in the field indicated by its title. A briel description of the activities, and of the resultant publications, appears on the inside of the front cover.

\section{IH ASHIVGTON, U. C.}

Electricity. Resistance and Reactance. Electrochemistry. Electrical lnstruments. Magnetic Measurements Dielectrics, lligh Voltage.

Metrology. Photometry and Colorimetry. Refractometry. Photographic Research. Length. Engineering Metrology. Mass and Scale. Volumetry and Densimetry.

Ileat. Temperature Physics. Heat Measurements. Cryogenic Physics. Equation of State. Statistical Physics. Radiation Physics. X-ray. Radioactivity. Radiation Theory. High Energy Radiation. Radiological Equipment. Nucleonic Instrumentation. Neutron Physics.

Analytical and Inorganic Chemistry. Pure Substances. Spectrochemistry. Solution Chemistry. Standard Reference Materials. Applied Analytical Research. Crystal Chemistry.

Mechanics. Sound. Pressure and Vacuum. Fluid Mechanics. Engineering Mechanics. Rheology. Combustion Controls.

Polsmers. Macromolecules: Synthesis and Structure. Polymer Chemistry. Polymer Physics. Polymer Characterization. Polymer Evaluation and Testing. Applied Polymer Standards and Research. Dental Research.

Metallurgy. Engineering Metallurgy. Microscopy and Diffraction. Metal Reactions. Metal Physics. Electrolysis and Metal Deposition.

Inorganic Solids. Engineering Ceramics. Glass. Solid State Chemistry. Crystal Growth. Physical Properties. Crystallography.

Building Research. Structural Engineering. Fire Research. Mechanical Systems. Organic Building Materials. Codes and Safety Standards. Heat Transfer. Inorganic Building Materials. Metallic Building Materials.

Applied Mathematics. Numerical Analysis. Computation. Statistical Engineering. Mathematical Physics. Operations Research.

Data Processing Systems. Components and Techniques. Computer Technology. Measurements Automation. Engineering Applications. Systems Analysis.

Atomic Physics. Spectroscopy. Infrared Spectroscopy. Far Illtraviolet Physics. Solid State Physics. Electron Physics. Atonic Physics. Plasma Spectroscopy.

Instrumentation. Engineering Electronics. Electron Devices. Electronic Instrumentation. Mechanical Instruments. Basic lnstrumentation.

Physical Chemistry. Thermochemistry. Surface Chemistry. Organic Chemistry. Molecular Spectroscopy. Elementary Processes. Mass Spectrometry. Photochemistry and Radiation Chemistry.

Office of Weights and Measures.

\section{BOULDER, COLO.}

Cryogenic Engineering Laboratory. Cryogenic Equipment. Cryogenic Processes. Properties of Materials. Cryogenic Technical Services.

\section{CENTRAL, RADIO PROPAGATION LABORATORY}

Ionosphere Research and Propagation. Low Frequency and Very Low Frequency Research. Ionosphere Research. Prediction Services. Sun-Earth Relationships. Field Engineering. Radio Warning Services. Vertical Soundings Research.

Radio Propagation Engineering. Data Reduction Instrumentation. Radio Noise. Tropospheric Measurements. Tropospheric Analysis. Propagation-Terrain Effects. Radio-Meteorology. Lower Atmosphere Physics.

Radio Systems. Applied Flectromagnetic Theory. High Frequency and Very High Frequency Research. Frequency Utilization. Modulation Research. Antenna Research. Radiodetermination.

Upper Atmosphere and Space Physics. Upper Atmosphere and Plasma Physics. High Latitude Ionosphere Physics. lonosphere and Exosphere Scatter. Airglow and Aurora. Ionospheric Radio Astronomy.

\section{RADIO STANDARDS LABORATORY}

Radio Physics. Radio Broadcast Service. Radio and Microwave Materials. Atomic Frequency and Time-Interval Standards. Radio Plasma. Millimeter-Wave Research.

Circuit Standards. High Frequency Electrical Standards. High Frequency Calibration Services. High Frequency lmpedance Standards. Microwave Calibration Services. Microwave Circuit Standards. Cow Frequency Calibration Services. 
NBS $>$ 